\title{
An integrative taxonomic revision of Slug-eating Snakes (Squamata: Pareidae: Pareineae) reveals unprecedented diversity in Indochina
}

\author{
Nikolay A Poyarkov ${ }^{\text {Corresp., } 1,2}$, Tan Van Nguyen ${ }^{3}$, Parinya Pawangkhanant ${ }^{4}$, Platon V. Yushchenko ${ }^{2}$, Peter Brakels ${ }^{5}$, \\ Linh Hoang Nguyen ${ }^{6}$, Hung Ngoc Nguyen ${ }^{6}$, Chatmongkon Suwannapoom ${ }^{4}$, Nikolai Orlov ${ }^{7}$, Gernot Vogel $^{8}$ \\ ${ }^{1}$ Laboratory of Tropical Ecology, Joint Russian-Vietnamese Tropical Research and Technological Center, Hanoi, Hanoi, Vietnam \\ 2 Faculty of Biology, Department of Vertebrate Zoology, Moscow State University, Moscow, Moscow, Russia \\ 3 Department of Species Conservation, Save Vietnam's Wildlife, Ninh Binh, Ninh Binh, Vietnam \\ 4 Division of Fishery, School of Agriculture and Natural Resources, University of Phayao, Phayao, Phayao, Thailand \\ 5 IUCN Laos PDR, Vientiane, Vientiane, Lao PDR \\ 6 Department of Zoology, Southern Institute of Ecology, Vietnam Academy of Science and Technology, Ho Chi Minh City, Ho Chi Minh City, Vietnam \\ 7 Department of Herpetology, Zoological Institute, Russian Academy of Sciences, St. Petersburg, St. Petersburg, Russia \\ 8 Society for Southeast Asian Herpetology, Heidelberg, Germany \\ Corresponding Author: Nikolay A Poyarkov \\ Email address: n.poyarkov@gmail.com
}

Slug-eating snakes of the subfamily Pareinae are an insufficiently studied group of snakes specialized in feeding on terrestrial mollusks. Currently Pareinae encompass three genera with 34 species distributed across the Oriental biogeographic region. Despite the recent significant progress in understanding of Pareinae diversity, the subfamily remains taxonomically challenging. Here we present an updated phylogeny of the subfamily with a comprehensive taxon sampling including 30 currently recognized Pareinae species and several previously unknown candidate species and lineages. Phylogenetic analyses of mtDNA and nuDNA data supported the monophyly of the three genera Asthenodipsas, Aplopeltura, and Pareas. Within both Asthenodipsas and Pareas our analyses recovered deep differentiation with each genus being represented by two morphologically diagnosable clades, which we treat as subgenera. We further apply an integrative taxonomic approach, including analyses of molecular and morphological data, along with examination of available type materials, to address the longstanding taxonomic questions of the subgenus Pareas, and reveal the high level of hidden diversity of these snakes in Indochina. We restrict the distribution of $P$. carinatus to southern Southeast Asia, and recognize two subspecies within it, including one new subspecies proposed for the populations from Thailand and Myanmar. We further revalidate $P$. berdmorei, synonymize $P$. menglaensis with $P$. berdmorei, and recognize three subspecies within this taxon, including the new subspecies erected for the populations from Laos and Vietnam. 
Furthermore, we describe two new species of Pareas from Vietnam: one belonging to the $P$. carinatus group from southern Vietnam, and a new member of the $P$. nuchalis group from the central Vietnam. We provide new data on $P$. temporalis, and report on a significant range extension for $P$. nuchalis. Our phylogeny, along with molecular clock and ancestral area analyses, reveal a complex diversification pattern of Pareinae involving a high degree of sympatry of widespread and endemic species. Our analyses support the "upstream" colonization hypothesis and, thus, the Pareinae appears to have originated in Sundaland during the middle Eocene and then colonized mainland Asia in early Oligocene. Sundaland and Eastern Indochina appear to have played the key roles as the centers of Pareinae diversification. Our results reveal that both vicariance and dispersal are responsible for current distribution patterns of Pareinae, with tectonic movements, orogeny and paleoclimatic shifts being the probable drivers of diversification. Our study brings the total number of Pareidae species to 41 and further highlights the importance of comprehensive taxonomic revisions not only for the better understanding of biodiversity and its evolution, but also for the elaboration of adequate conservation actions. 
An integrative taxonomic revision of Slug-eating Snakes (Squamata: Pareidae: Pareineae) reveals unprecedented diversity in Indochina

Nikolay A. Poyarkov ${ }^{1,2, *}$, Tan Van Nguyen $^{3}$, Parinya Pawangkhanant ${ }^{4}$, Platon V. Yushchenko $^{1}$, Peter Brakels ${ }^{5}$, Linh Hoang Nguyen ${ }^{6}$, Hung Ngoc Nguyen ${ }^{6}$, Chatmongkon

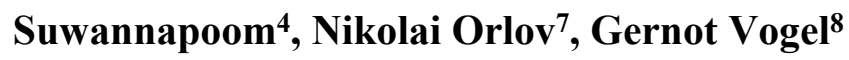

1 Faculty of Biology, Department of Vertebrate Zoology, Moscow State University, Moscow, Moscow, Russia

2 Laboratory of Tropical Ecology, Joint Russian-Vietnamese Tropical Research and Technological Center, Hanoi, Hanoi, Vietnam

${ }^{3}$ Department of Species Conservation, Save Vietnam's Wildlife, Ninh Binh, Ninh Binh, Vietnam

${ }^{4}$ Division of Fishery, School of Agriculture and Natural Resources, University of Phayao, Phayao, Phayao, Thailand

${ }^{5}$ IUCN Laos PDR, Vientiane, Vientiane, Lao PDR

${ }^{6}$ Department of Zoology, Southern Institute of Ecology, Vietnam Academy of Science and Technology, Ho Chi Minh City, Ho Chi Minh City, Vietnam

${ }^{7}$ Department of Herpetology, Zoological Institute, Russian Academy of Sciences, St. Petersburg, St. Petersburg, Russia

${ }^{8}$ Society for Southeast Asian Herpetology, Heidelberg, Germany

* Corresponding author: Nikolay A. Poyarkov: n.poyarkov@gmail.com.

\section{ABSTRACT} specialized in feeding on terrestrial mollusks. Currently Pareinae encompass three genera with 34 species distributed across the Oriental biogeographic region. Despite the recent significant progress in understanding of Pareinae diversity, the subfamily remains taxonomically challenging. Here we present an updated phylogeny of the subfamily with a comprehensive taxon sampling including 30 currently recognized Pareinae species and several previously unknown 
32 candidate species and lineages. Phylogenetic analyses of mtDNA and nuDNA data supported the monophyly of the three genera Asthenodipsas, Aplopeltura, and Pareas. Within both Asthenodipsas and Pareas our analyses recovered deep differentiation with each genus being represented by two morphologically diagnosable clades, which we treat as subgenera. We further apply an integrative taxonomic approach, including analyses of molecular and morphological data, along with examination of available type materials, to address the longstanding taxonomic questions of the subgenus Pareas, and reveal the high level of hidden diversity of these snakes in Indochina. We restrict the distribution of $P$. carinatus to southern Southeast Asia, and recognize two subspecies within it, including one new subspecies proposed for the populations from Thailand and Myanmar. We further revalidate $P$. berdmorei, synonymize $P$. menglaensis with $P$. berdmorei, and recognize three subspecies within this taxon, including the new subspecies erected for the populations from Laos and Vietnam. Furthermore, we describe two new species of Pareas from Vietnam: one belonging to the P. carinatus group from southern Vietnam, and a new member of the $P$. nuchalis group from the central Vietnam. We provide new data on $P$. temporalis, and report on a significant range extension for P. nuchalis. Our phylogeny, along with molecular clock and ancestral area analyses, reveal a complex diversification pattern of Pareinae involving a high degree of sympatry of widespread and endemic species. Our analyses support the "upstream" colonization hypothesis and, thus, the Pareinae appears to have originated in Sundaland during the middle Eocene and then colonized mainland Asia in early Oligocene. Sundaland and Eastern Indochina appear to have played the key roles as the centers of Pareinae diversification. Our results reveal that both vicariance and dispersal are responsible for current distribution patterns of Pareinae, with tectonic movements, orogeny and paleoclimatic shifts being the probable drivers of diversification. Our study brings the total number of Pareidae species to 41 and further highlights the importance of comprehensive taxonomic revisions not only for the better understanding of biodiversity and its evolution, but also for the elaboration of adequate conservation actions.

Subjects: Biodiversity, Biogeography, Evolutionary Studies, Taxonomy, Zoology

Keywords: Pareas, Asthenodipsas, Aplopeltura, Eberhardtia, Spondylodipsas, molecular phylogeny, biogeography, Southeast Asia, Sundaland, cryptic species 


\section{INTRODUCTION}

65

The snakes of the family Pareidae Romer, 1956 (Squamata, Serpentes) currently (as of December $\left.1^{\text {st }}, 2021\right)$ encompassing 39 species inhabiting the Oriental biogeographic region are divided into two subfamilies: Pareinae Romer, 1956 in Southeast Asia and Xylophiinae Deepak, Ruane \& Gower, 2019 in southern India (Deepak et al., 2019; Uetz, Freed \& Hošek, 2021). Slugeating snakes (or snail-eating snakes) of the subfamily Pareinae are widely distributed throughout the tropical and subtropical areas of Southeast and East Asia. Its members are mainly smallsized, arboreal, nocturnal snakes, and are regarded as dietary specialists of terrestrial pulmonates i.e., slugs and snails (You, Poyarkov \& Lin, 2015; Cundall \& Greene, 2000). Snail-eating species of Pareinae are unique among terrestrial vertebrates in having asymmetric lower jaws, with more teeth on the right mandible than on the left (Hoso et al., 2007, 2010). Due to the specialized feeding habit and foraging behaviour, the evolutionary biology of Pareas has received much attention in recent years (Götz, 2002; Hoso \& Hori, 2006, 2008; Hoso, 2007; Hoso et al., 2007, 2010; You, Poyarkov \& Lin, 2015; Danaisawadi et al., 2015, 2016; Kojima et al., 2020; Chang et al., 2021).

The subfamily Pareinae had a turbulent taxonomic history (David \& Vogel, 1996; Rao \& Yang, 1992) with recent works (Grossmann \& Tillack, 2003; Guo et al., 2011; Ding et al., 2020; Vogel et al., 2020, 2021) recognizing three genera: Pareas Wagler, 1830 with 24 species (type species: Pareas carinatus Wagler, 1830); Asthenodipsas Peters, 1864 with nine species (type species: Asthenodipsas malaccana [Peters, 1864]), and a monotypic genus Aplopeltura Duméril, 1853 (type species: Aplopeltura boa [Boie, 1828]). Two genus-level nomens, namely Eberhardtia Angel, 1920 (type species: Eberhardtia tonkinensis Angel, 1920, regarded as a synonym of Pareas formosensis [Van Denburgh, 1909] by Ding et al., 2020) and Internatus Yang \& Rao, 1992 (type species: Asthenodipsas leavis [Boie, 1827]) are presently considered junior synonyms of the genera Pareas and Asthenodipsas, respectively (see Grosmann \& Tillack, 2003; Wallach, Williams \& Boundi, 2014; Ding et al., 2020; Vogel et al., 2020, 2021). Several recent phylogenetic studies suggested that the genus Pareas consists of two highly divergent major clades and is paraphyletic with respect to Aplopeltura or Asthenodipsas (Guo et al., 2011; Pyron et al., 2011; Wang et al., 2020). At the same time, other multilocus studies recovered 
94 Pareas as a monophyletic group though with moderate or low node support values, and 95 suggested the genus Aplopeltura as its sister taxon (Pyron, Burbrink \& Wiens, 2013; You, Poyarkov \& Lin, 2015; Figueroa et al., 2016; Deepak et al., 2019; Zaher et al., 2019). The genus Asthenodipsas was also shown to include two major lineages (Loredo et al., 2013; Figueroa et al., 2016; Deepak et al., 2019; Wang et al., 2020), though its monophyly got only moderate support based on the concatenated analysis of mitochondrial and nuclear DNA markers (Wang et al., 2020; Ding et al., 2020; Vogel et al., 2021). Therefore, despite the recent significant progress in evolutionary studies on Pareinae, the phylogenetic relationship among the major genus-level lineages of the subfamily still remain debated and unclear.

Several recent taxonomic studies have demonstrated that the species diversity of Pareinae is still underestimated (e.g., Vogel, 2015; Hauser, 2017; Quah et al., 2019, 2020, 2021; Le et al., 2021). The high degree of morphological similarity among closely related taxa of Pareinae often makes species delineation in slug snakes quite challenging (Guo \& Deng, 2009; Vogel, 2015; Yang et al., 2021), suggesting that the molecular data represent an effective tool to help untangle taxonomic controversies when morphological analyses yield inconsistent results (You, Poyarkov \& Lin, 2015; Loredo et al., 2013; Vogel et al., 2020, 2021; Bhosale et al., 2020; Wang et al., 2020; Ding et al., 2020; Liu \& Rao, 2021; Yang et al., 2021). Application of the integrative taxonomic approach combining evidence from morphological and molecular data resulted in the discovery of several previously unnoticed taxa and allowed to revise several species complexes, including the Pareas hamptoni complex (You, Poyarkov \& Lin, 2015; Bhosale et al., 2020; Ding et al., 2020; Liu \& Rao, 2021; Yang et al., 2021), the P. margaritophorus complex (Vogel et al., 2020; Suntrarachun et al., 2020), and the P. monticola complex (Vogel et al., 2021).

On the other hand, the Keeled slug snake, Pareas carinatus, has received comparatively little attention in most recent revisions. This species was originally described by Wagler (1830) from Java, Indonesia, and was later reported to be widely distributed throughout Southeast Asia, from southern China, southern Myanmar, Laos, south-western and eastern Cambodia, Vietnam, Thailand, southwards to Peninsular Malaysia, and islands of Borneo, Sumatra, Java and Bali (Wallach, Williams \& Boundi, 2014). However, since geographic variation of this species has never been examined across the different regions, its taxonomic status remained controversial and a number of misidentifications were made in the past (e.g., see discussion in Das, 2012, 2018). Recently, Wang et al. (2020) demonstrated P. nuchalis (Boulenger, 1900) to be closely 
125 related to $P$. carinatus complex, and divided the latter by describing $P$. menglaensis Wang, Che,

126 Liu, Li, Jin, Jiang, Shi \& Guo, 2020, as a sister species of P. carinatus sensu stricto. However, in

127 this revision the authors did not examine type specimens of $P$. carinatus, and also have neglected

128 to re-evaluate the status of two available species names currently considered as junior synonyms

129 of P. carinatus: Pareas berdmorei Theobald, 1868, and Amblycephalus carinatus unicolor 130 Bourret, 1934 (see Nguyen, Ho \& Nguyen, 2009; Wallach, Williams \& Boundi, 2014; Uetz,

131 Freed \& Hošek, 2021). The most recent addition to the taxonomy of the group is the discovery of

132 a new species from southern Vietnam - P. temporalis, which was suggested as a sister species to 133 P. nuchalis from Borneo (Le et al., 2021); the authors also provided the most complete 134 phylogeny for the genus Pareas published up to date, generally concordant with the earlier 135 results (Wang et al., 2020; Vogel et al., 2021). The taxonomic history of the P. carinatus - P. 136 nuchalis complex is summarized in Table 1. Overall, the taxonomic status of P. carinatus, its 137 synonyms, and $P$. menglaensis remains unclear pending an integrative study combining data on 138 molecular and morphological variation of this group throughout its range.

139 In the present study, we provide an updated phylogeny for the subfamily Pareinae based on 140 the analysis of mitochondrial and nuclear DNA markers, and re-assess the genus-level taxonomy of the group. Based on an extensive sampling we also report on a previously unrecognized diversity of the genus Pareas in Indochina. We examine name-bearing types and re-assess taxonomy of the $P$. carinatus complex using an integrative taxonomic approach, combining morphological and molecular data from the newly collected and older specimens preserved in herpetological collections. We also provide an updated identification key for the members of the subfamily Pareinae and species of the $P$. carinatus complex. Finally, we conduct a divergence time estimation analysis for the subfamily Pareinae and discuss evolution and the historical biogeography of this peculiar group of snakes.

\section{MATERIALS AND METHODS}

\section{Species concept}

153

154

In the present study, we follow the General Lineage Concept (GLC: De Queiroz, 2007) which suggests that a species constitutes a population of organisms independently evolving from 155 other such populations owing to a lack of gene flow (Barraclough et al., 2003; De Queiroz, 
156

157

158

159

160

161

162

163

164

165

166

167

168

169

170

171

172

173

174

175

176

177

178

179

180

181

182

183

184

185

186

2007). Numerous recent integrative taxonomic studies, rather than relying solely on traditional taxonomic procedure increasingly, use a wide range of empirical data to delimit species boundaries (reviewed in Coyne \& Orr, 1998; Knowles \& Carstens, 2007; Fontaneto et al., 2007). We herein follow the framework of integrative taxonomy (Padial et al., 2010; Vences et al., 2013) which relies on independent multiple lines of evidence to assess the taxonomic status of the lineages. To infer species boundaries we use the DNA-based molecular phylogenies, while to describe those boundaries we rely on univariate (ANOVA) and multivariate (PCA) morphological analyses (e.g. Okamiya et al., 2018).

\section{Nomenclatural acts}

The electronic version of this article in Portable Document Format (PDF) will represent a published work according to the International Commission on Zoological Nomenclature (ICZN), and hence the new names contained in the electronic version are effectively published under that Code from the electronic edition alone (see Articles 8.5-8.6 of the Code). This published work and the nomenclatural acts it contains have been registered in ZooBank, the online registration system for the ICZN. The ZooBank Life Science Identifiers (LSIDs) can be resolved and the associated information can be viewed through any standard web browser by appending the LSID to the prefix http://zoobank.org/.

The LSID for this publication is as follows: urn:lsid:zoobank.org:pub:192CDD83-E08C40B1-92EB-3DB2C3E63CFA. The online version of this work is archived and available from the following digital repositories: PeerJ, PubMed Central and CLOCKSS.

\section{Taxon sampling}

We used tissues from the herpetological collections of Zoological Museum of Moscow University (ZMMU; Moscow, Russia); California Academy of Sciences Museum (CAS; California, USA); Southern Institute of Ecology Zoological Collection (SIEZC; Ho Chi Minh City, Vietnam); School of Agriculture and Natural Resources, University of Phayao (AUP; Phayao, Thailand); and National Museum of Natural Science (NMNS, Taichung, Taiwan) (summarized in Supplementary Table S1 and Appendix I). For alcohol-preserved voucher specimens stored in museum collections, we removed a small sub-sample of muscle, preserved it in $96 \%$ ethanol, and stored samples at $-70{ }^{\circ} \mathrm{C}$. Altogether, we analyzed 48 tissue samples 
187 representing 20 nominal taxa of the genus Pareas. Geographic location of sampled populations 188 of the members of the subgenus Pareas is presented in Fig. 1.

189 Permissions to conduct fieldwork and collect specimens were granted by the Department of 190 Forestry, Ministry of Agriculture and Rural Development of Vietnam (permit numbers 191 \#547/TCLN-BTTN; \#432/TCLN-BTTN; \#822/TCLN-BTTN; \#142/SNgV-VP; \#1539/TCLN192 DDPH, \#1700/UBND.VX); the Forest Protection Departments of the Peoples' Committees of 193 Gia Lai Province (permit numbers \#530/UBND-NC; \#1951/UBND-NV), Phu Yen Province 194 (permit number \#05/UBND-KT); Phu Tho Province (permit number \#2394/UBND-TH3); Thanh 195 196 Hoa Province (permit number \#3532/UBND-THKH); and Quang Nam Province (permit number \#308/SNgV-LS), Vietnam; by the Biotechnology and Ecology Institute Ministry of Science and Technology, Lao PDR (permit no. 299); and by the Institute of Animals for Scientific Purpose Development (IAD), Bangkok, Thailand (permit numbers U1-01205-2558 and UP-AE59-01-040022). Specimen collection protocols and animal operations followed the Institutional Ethical Committee of Animal Experimentation of University of Phayao (permit number 610104022).

201

202

\section{DNA isolation, PCR, and sequencing}

To infer the phylogenetic relationships among the Pareinae we obtained partial sequence 204 data of cytochrome $b$ (cyt $b$ ) and NADH dehydrogenase subunit 4 (ND4) mtDNA genes, as well as two nuclear genes: oocyte maturation factor mos $(c-m o s)$ and recombination activating gene 1 $(R A G 1)$. These genetic markers have been widely applied in studies of Pareidae diversity and phylogenetic relationships (e.g., Guo et al., 2011; You, Poyarkov \& Lin, 2015; Deepak et al., 2019; Wang et al., 2020; Vogel et al., 2020, 2021; Ding et al., 2020). Total genomic DNA was extracted from muscle or liver tissue samples preserved in $95 \%$ ethanol using standard phenolchloroform-proteinase $\mathrm{K}$ (final concentration $1 \mathrm{mg} / \mathrm{ml}$ ) extraction procedures with consequent isopropanol precipitation (protocols followed Russell \& Sambrook, 2001). DNA amplification was performed in $20 \mathrm{ml}$ reactions using ca. $50 \mathrm{ng}$ genomic DNA, $10 \mathrm{nmol}$ of each primer, 15 nmol of each dNTP, $50 \mathrm{nmol}$ of additional $\mathrm{MgCl}_{2}$, Taq PCR buffer (10 mM of Tris- $\mathrm{HCl}, \mathrm{pH} 8.3$, $50 \mathrm{mM}$ of $\mathrm{KCl}, 1.1 \mathrm{mM}$ of $\mathrm{MgCl}_{2}$, and $0.01 \%$ gelatine) and $1 \mathrm{U}$ of Taq DNA polymerase. Primers used for PCR and sequencing are summarized in Supplementary Table S2. PCRs were run on a Bio-Rad T100TM Thermal Cycler. PCR protocols for cyt $b$ and ND4 gene fragments 
$218 c$-mos gene were identical to those described in Slowinski \& Lawson (2002), and for RAG1 to

219 those described in Groth \& Barrowclough (1999) and Chiari et al. (2004). Sequence data 220 collection and visualization were performed on an ABI 3730xl automated sequencer (Applied 221 Biosystems, Foster City, CA, USA). PCR purification and cycle sequencing were done 222 commercially through Evrogen Inc. (Moscow, Russia).

223

(DNASTAR Inc., Madison, WI, USA), MEGA 7 (Kumar, Stecher \& Tamura, 2016), and BioEdit v7.0.5.2 (Hall, 1999). For individuals which were detected to be heterozygous in nuclear gene sequences, they were phased using the software program PHASE with default sets of iterations, burn-in, and threshold (Stephens et al., 2001), on the web-server interface SEQPHASE (Flot, 2010). One of the phased copies was selected at random to represent each individual in subsequent analyses. All sequences were deposited in GenBank (see Supplementary Table S1 for accession numbers).

To reconstruct the phylogenetic relationships within the Pareinae, we aligned the newly obtained cyt $b, N D 4, c$-mos, and $R A G 1$ sequences together with representative sequences from 32 specimens of approximately 16 nominal Pareas species and seven other Pareinae representatives, retrieved from GenBank (see Supplementary Table S1). Two species of the genus Xylophis (Pareidae: Xylophinae) were added to the alignment and used as outgroups for rooting the phylogenetic tree following the phylogenetic data of Deepak et al. (2019, 2020). In total, we obtained molecular genetic data for 81 samples representing 38 taxa of Pareinae, including all currently recognized species of the genus Pareas, five species of Asthenodipsas, and the single species of the genus Aplopeltura (A. boa). Details on taxonomy, localities, GenBank accession numbers, and associated references for all examined specimens are summarized in Supplementary Table S1.

The nucleotide sequences were initially aligned in MAFFT v.6 (Katoh et al., 2002) with default parameters; the alignment was subsequently checked by eye in BioEdit 7.0.5.2 (Hall, 1999) and slightly adjusted. The mean uncorrected genetic $p$-distances between sequences were calculated with MEGA 7 (Kumar, Stecher \& Tamura, 2016). Phylogenetic trees were estimated 
$249 R A G 1)$ datasets. The total evidence analysis was performed as the approximately unbiased tree250 selection test (AU-test; Shimodaira, 2002) conducted using Treefinder v.March 2011 (Jobb, 251 2011) did not reveal statistically significant differences between mtDNA and nuDNA topologies.

252 Phylogenetic relationships of Pareinae were inferred using Bayesian Inference (BI) and 253 Maximum Likelihood (ML) approaches. The optimum partitioning schemes for alignments were 254 identified with PartitionFinder 2.1 .1 (Lanfear et al., 2012) using the greedy search algorithm 255 under an AIC criterion, and are presented in Supplementary Table S3. When the same model was 256 proposed to different codon positions of a given gene, they were treated as a single partition.

257 BI was performed in MrBayes v3.1.2 (Ronquist \& Huelsenbeck, 2003) with two 258 simultaneous runs, each with one cold chain and three heated chains for 200 million generations. 259 Two independent Metropolis-coupled Markov chain Monte Carlo (MCMCMC) runs were 260 performed and checked for the effective sample sizes (ESS) were all above 200 by exploring the 261 likelihood plots using TRACER v1.6 (Rambaut \& Drummond, 2007). We discarded the initial $26210 \%$ of trees as burn-in. Confidence in tree topology was assessed by posterior probability for 263 Bayesian analysis (BI PP) (Huelsenbeck \& Ronquist, 2001). Nodes with BI PP values of 0.95 and 264 above were considered strongly supported, nodes with values of 0.90-0.94 were considered as 265 well-supported, and the BI PP values below 0.90 were regarded as no support (Wilcox et al., 266 2002).

A Maximum Likelihood (ML) analysis was implemented using the IQ-TREE webserver (Nguyen et al., 2015; Trifinopoulos et al., 2016). One-thousand bootstrap pseudoreplicates via the ultrafast bootstrap (ML UB; Hoang et al., 2018) approximation algorithm were employed, and nodes having ML UB values of $95 \%$ and above were considered strongly supported, while nodes with values of $90 \%-94 \%$ we regarded as well-supported, and the ML UB node values below 90\% were considered as no support (Minh et al., 2013).

\section{Divergence times estimation}

The time-calibrated Bayesian Inference analysis was implemented in the program Bayesian Evolutionary Analysis Utility (BEAUti) version 2.4.7 and run on BEAST v1.8.4 (Drummond et al., 2012), including the concatenated mtDNA + nuDNA dataset. We used hierarchical likelihood ratio tests in PAML v4.7 (Yang, 2007) to test molecular clock assumptions separately for mtDNA and nuDNA markers. Based on PAML results, which indicated that there was very little 
280 rate variation among the sites of mtDNA markers and so a strict clock model was used for the

281 final analysis employing unlinked site and linked tree models for the nuDNA, and an 282 uncorrelated lognormal relaxed clock for mtDNA genes. We also used these models and 283 partitioning schemes from the ML analysis with empirical frequencies estimated so as to fix them 284 to the proportions observed in the data. A coalescent exponential population prior was employed 285 as the tree prior because intraspecific relationships among many individuals were being assessed 286 and it was not known a priori which individuals would be grouped as species. Under the 287 coalescent model, the default priors for population growth (Laplace Distribution) and size (1/X) 288 were left unchanged because these parameters were not being estimated. We conducted two runs 289 of 100 million generations each in BEAST v1.8.4. We also assumed parameter convergence in 290 Tracer and discarded the first $10 \%$ of generations as burn-in. We used TreeAnnotator v1.8.0 (in 291 BEAST) to create our maximum credibility clades. Since no paleontological data for the Pareidae 292 are known to exist, we relied on four recently estimated calibration priors for this family obtained 293 from recent large-scale phylogeny of the group (Deepak et al., 2019) as primary calibration 294 295 points. Calibration points and priors are summarized in Supplementary Table S4. testing approach in a common ML framework to find the best statistical fit using AIC in RASP v3.2 (Ree et al., 2005; Ree \& Smith, 2008; Yu et al., 2015). The models allow testing alternative biogeographic hypotheses, such as dispersal, vicariance, and extinction. Six areas were defined that are covered by our ingroup sample (see Fig. 2A): (A) Mainland East Asia; (B) Eastern Indochina; (C) Western Indochina; (D) Indo-Burma, including eastern Himalaya and the Arakan Mountains of Myanmar; (E) Sundaland; and (F) East Asian islands (Taiwan + the Ryukyus) following Gorin et al. (2020), Chen et al. (2018), and Nguyen et al. (2020a). This coding scheme reflects the complex palaeogeographic history of Southeast Asia, because Borneo, Java, Sumatra and the Thai-Malay Peninsula constituted the connected landmass of Sundaland until recently (Hall, 2012; Morley, 2018). Maximum areas per species were set to three, as no extant species occurs in more than three biogeographical regions. Matrices of modern distributions of species across the areas are presented in Supplementary Table S5; transition matrices between biogeographic regions are given in Supplementary Table S6. Discrete state transitions for ranges 
311 were estimated using ML framework on branches as functions of time, suggesting the best fit

312 model for ancestral ranges at the times of cladogenesis using the Akaike Information Criterion

313 (AIC) and Akaike weights (Ree \& Smith, 2008; Matzke, 2013). Two models were compared:

314 Langrange Dispersal-Extinction-Cladogenesis (DEC; Ree \& Smith, 2008), and the ML version of

315 Statistical Dispersal-Vicariance Analysis (S-DIVA; Ronquist, 1997).

316

317

318

319

320

321

322

(following Vogel, 2015). Morphological measurements (all in mm) included: snout-vent length

323 (SVL); tail length (TaL); total length (TL); relative tail length (TaL/TL); horizontal eye diameter (ED); distance from the anterior edge of orbit to nostril (Eye-nos); minimal distance from the ventral edge of orbit to the edge of upper lip (Eye-mouth); head length from snout tip to jaw angles (HL); maximal head width (HW). Meristic characters evaluated were the number of dorsal scale rows counted at one head length behind head (ASR), at mid-body (MSR), namely at $\mathrm{SVL} / 2$, and at one head length before vent (PSR); number of enlarged vertebral scale rows (VSE); presence of keeled dorsal scale rows (DORkeel); number of keeled dorsal scale rows at midbody (KMD); number of ventral scales (VEN); number of preventral scales (preVEN); number of subcaudal scales (SC); number of cloacal (anal) plates (AN); number of supralabials (SL); number of supralabials touching the orbit (SL-eye); number of supralabials touching subocular (SL-suboc); number of infralabials (IL); numbers of infralabials touching each other (IL-touch); number of nasals (NAS); number of anterior temporals (At); number of posterior temporals $(\mathrm{Pt})$; number of loreals (LOR); loreal touching the orbit or not (LOR-eye); number of preoculars (Preoc); number of presuboculars (Presuboc); prefrontal touching the orbit or not (Prefr-eye); number of suboculars (SoO); subocular fused with postocular or not (SoO-PoO); number of postoculars ( $\mathrm{PoO})$. Coloration and pattern characters evaluated were the background body dorsal coloration; presence or absence of ornamentation on neck; presence or absence of dark blotches or chevron on neck and nuchal areas; coloration of head dorsal surface; presence and number of postorbital stripes; presence or absence of a dark blotch on $7^{\text {th }}$ supralabial; 
342 presence or absence of transverse bands on body; number of transverse bands on body; number

343 of discontinuous dorsal bands comprised of dark dots; presence or absence of body 344 ornamentation others than bands; dorsal bands continue on belly or not; belly pattern (no pattern, 345 banded, mottled or dotted).

346 We took the color notes from living specimens or their digital images prior to preservation 347 following Vogel (2015). We measured body and tail lengths with a measuring tape (to the nearest 348 of $1 \mathrm{~mm}$ ); all other measurements were taken using a Mitutoyo digital slide-caliper (to the 349 nearest $0.1 \mathrm{~mm}$ ). We counted the number of ventral scales following Dowling (1951); we 350 regarded the first enlarged shield anterior to the ventrals as a preventral, while half-ventrals were 351 counted as one. We regarded the first scale under the tail contacting its opposite as the first 352 subcaudal. We did not include the terminal tail scute in the number of subcaudals. Supralabial(s) 353 touching the presubocular were included in the SL-suboc count. We regarded as infralabials 354 those shields that were bordering the mouth gap and were placed ventrally than supralabials. 355 Smaller shields located posteriorly than the last enlarged supralabial shield do not border the 356 mouth gap and were excluded in the sublabial scales count. We defined the scale starting 357 between the posterior chin shield and the infralabials and bordering the infralabials as the first 358 sublabial shield. We recorded the values for paired head characters on both sides of the head (in a 359 left / right order). We determined the sex of the specimens by the presence of everted hemipenes 360 or by dissection of the ventral tail base. We described the morphology of hemipenial structures on specimens in which such structures were everted before preservation; description and terminology followed Keogh (1999).

An analysis of variance (ANOVA) was performed to ascertain if statistically significant mean differences among meristic characters $(p<0.05)$ existed among the discrete populations delimited in the phylogenetic analyses. ANOVAs having a p-value less than 0.05 indicating that statistical differences existed were subjected to a Tukey HSD test to ascertain which population pairs differed significantly $(\mathrm{p}<0.05)$ from each other. We used the Principal Component Analysis (PCA) to determine if populations from different localities occupied unique positions in morphospace, as well as the degree to which their variation coincided with potential species boundaries as predicted by the molecular phylogeny and univariate analyses. Juvenile specimens, as well as the specimens with incomplete or damaged tails were excluded from the PCA. 
373 Eye-mouth, HL, and HW, and the discrete meristic data from the scale counts VSE, KMD, 374 DORkeel, VEN, preVEN, SC, SL, SL-eye, IL, At, Pt, LOR, Preoc, Presuboc, Prefr-eye, SoO, 375 SoO-PoO, and PoO. In order to normalize the PCA data distribution and to transform meristic 376 and mensural data into comparable units for analysis, we natural log-transformed all PCA data 377 prior to analysis and scaled it to their standard deviation. To exclude possible overweighting 378 effects, when we found a high correlation between certain pairs of characters, we omitted one of 379 them from the analyses. Statistical analyses were carried out using Statistica 8.0 (Version 8.0; 380 StatSoft, Tulsa, OK, USA).

381 Morphological and coloration characters of the examined specimens were compared in 382 detail to other species of the genus the Pareas. The examined comparative material is listed in 383 Appendix II. For comparison with other taxa, we also relied on previously published data (e.g., 384 Theobald, 1868b; Bourret, 1934; Pope, 1935; Smith, 1943; Taylor, 1965; Guo \& Rao, 2004; 385 Guo \& Deng, 2009; Stuebing et al., 2014; You, Poyarkov \& Lin, 2015, Vogel, 2015; Hauser, 386 2017; Wang et al., 2020; Vogel et al., 2020, 2021; Ding et al., 2020; Bhosale et al., 2020; Liu \& 387 Rao, 2021; Le et al., 2021). Other abbreviations used: Prov.: Province; Mt.: Mountain; N.P.: 388 National Park; N.R. Natural Reserve; Is.: Island; asl: above sea level.

\section{RESULTS}

391

\section{Partitions, substitution models, and sequence characteristics}

Our combined dataset was composed of $1,804 \mathrm{bp}$ of cyt $b$ and ND4 mtDNA genes, 1,757 394 bp of nuDNA (including $734 \mathrm{bp}$ of $c-m o s$, and 1,023 bp of RAG1), and 3,561 bp (mtDNA + nuDNA), respectively. The concatenated mtDNA + nuDNA dataset included 81 samples, representing ca. 29 Pareas taxa, including all 24 currently recognized species of the genus (Uetz, Freed \& Hošek, 2021), one species of the monotypic genus Aplopeltura, five species of the genus Asthenodipsas (of nine currently recognized species, 56\%), and two outgroup taxa (see Supplementary Table S1). Information on fragment lengths and variability is summarized in models which resulted in nine partitions in total (Supplementary Table S3).

\section{Phylogenetic relationships and distribution}


404

405

406

407

408

409

410

411

412

413

414

415

416

417

418

419

420

421

422

423

424

425

426

427

428

429

430

431

432

433

434

Phylogenetic trees obtained with ML and BI analyses of the three data partitions (mtDNA + nuDNA, mtDNA, nuDNA) are congruent apart from the generally lower resolution of nuDNA trees (see Supplementary Figures S2-S3). Overall, since the mtDNA + nuDNA phylogenetic tree was mostly better resolved and had greater node support than the mtDNA and nuDNA trees, we relied on the combined mtDNA + nuDNA topology for inferring phylogenetic relationships and biogeographic history of Pareinae. The BI tree resulted from the analysis of the concatenated mtDNA + nuDNA data (Fig. 3) inferred the following set of phylogenetic relationships:

1) The subfamily Pareinae was subdivided into five major strongly supported, deeply divergent groups, including two groups within the genus Pareas sensu lato (clades A and B, see Fig. 3), the genus Aplopeltura (clade C, see Fig. 3), and two groups corresponding to the genus Asthenodipsas sensu lato (clades D and E, see Fig. 3).

2) The monophyly of the genus Asthenodipsas got strong support in mtDNA + nuDNA analysis (1.0/100; hereafter node support values are given for BI PP/ML UB, respectively; see Fig. 3), while it was rendered paraphyletic in the analysis of the mtDNA dataset alone, though with no significant node support. The two clades within Asthenodipsas correspond to the A. malaccana species group (clade E, in our analysis represented by $A$. laevis and A. borneensis; 1.0/100), and to the A. vertebralis species group (clade D, in our analysis including $A$. vertebralis, $A$. tropidonota, and $A$. lasgalenensis; 1.0/100).

3) The monophyly of the clade joining Pareas + Aplopeltura was strongly supported (1.0/99). The monotypic genus Aplopeltura (1.0/100) in our analysis was represented with two samples of $A$. boa from Peninsular Malaysia and Borneo (Sabah, Malaysia), which were assigned into two highly divergent lineages (see Fig. 3).

4) The monophyly of the genus Pareas sensu lato was strongly supported by all analyses (1.0/99); the genus comprised two reciprocally monophyletic highly supported groups: clade A, including the members of the P. carinatus - P. nuchalis complex (1.0/100); and clade B, including the remainder of Pareas species (1.0/100) (see Fig. 3).

5) Within the clade B, encompassing the majority of the genus Pareas diversity, four subclades were recovered corresponding to the following species groups:

a. Pareas hamptoni species group (subclade B1; 1.0/100) including $P$. formosensis, $P$. xuelinensis, $P$. geminatus, $P$. hamptoni, $P$. niger, $P$. 
435

436

437

438

439

440

441

442

443

444

445

446

447

448

449

450

451

452

453

454

455

456

457

458

459

460

461

462

463

464

465 mengziensis, P. iwasakii, P. atayal, P. komaii, P. vindumi, P. kaduri, and P. nigriceps. Pareas kaduri and P. nigriceps from East Himalaya formed a wellsupported monophylum (1.0/99). The three species of Pareas from the East Asian Islands also formed a well-supported clade (1.0/100); with P. komaii reconstructed as a sister species with respect to $P$. atayal $+P$. iwasakii though with a low nodal support $(0.56 / 88)$. Phylogenetic position of $P$. vindumi from Myanmar within the subclade B1 remained essentially unresolved (Fig. 3). The remaining species of the subclade B1 formed a well-supported clade, corresponding to the $P$. hamptoni species complex (1.0/100). Within the latter, $P$. niger and $P$. mengziensis from Yunnan Province of China grouped together $(1.0 / 100)$ and were represented with almost identical haplotypes. Pareas hamptoni from Myanmar and Northern Indochina was suggested as a sister taxon with respect to the clade joining $P$. xuelinensis from Yunnan and $P$. geminatus from Northern Indochina; the latter species was recovered as paraphyletic with respect to $P$. xuelinensis (1.0/100). Pareas formosensis was represented in our analysis with five major lineages from Taiwan and Hainan islands, southern mainland China and Eastern Indochina; the sample of topotype P. tonkinensis from northern Vietnam was placed within the P. formosensis radiation with strong support (0.99/98; see Fig. 3).

b. Pareas margaritophorus species group (subclade B2; 1.0/100) included four species from Indochina and Indo-Burma: $P$. andersonii, $P$. modestus, $P$. macularius, and P. margaritophorus. Pareas andersonii and P. modestus from Myanmar and Northeast India formed a well-supported clade (1.0/100), to which P. macularius (1.0/100) was recovered as a sister taxon. The latter species was represented in our analysis with two samples from Myanmar and Laos, which were assigned into two highly divergent lineages (see Fig. 3). Subclade B2 was suggested as a sister lineage with respect to subclade B1 though with significant nodal support (0.99/89; see Fig. 3).

c. Pareas chinensis species group (subclade B3; 1.0/100) included P. stanleyi, $P$. boulengeri, and $P$. chinensis from mainland China; the latter two species formed a strongly supported monophyletic group (1.0/100). Subclade B3 was 
466

467

468

469

470

471

472

473

474

475

476

477

478

479

480

481

482

483

484

485

486

487

488

489

490

491

492

493

494

495

496

suggested as a sister lineage with respect to the clade joining B1+B2 with strong nodal support (1.0/92; see Fig. 3).

d. Pareas monticola species group (subclade B4; 1.0/100) included two species from East Himalaya and Indo-Burma: P. monticola and P. victorianus. Subclade B4 was suggested as a sister taxon with respect to other species groups B1-B3 with strong node support (1.0/100; see Fig. 3).

6) Within the remainder of the genus Pareas (clade A; Fig. 3), unexpectedly high numbers of divergent evolutionary lineages were detected. Present taxonomy recognizes three species within this group: P. carinatus, P. menglaensis and P. nuchalis. Altogether, nine divergent lineages were distinguished by robust BI PP and ML UB node support in analyses of the combined mtDNA + nuDNA dataset (Fig. 3). Of these lineages, those which are presently assigned to $P$. carinatus, were recovered as paraphyletic with respect to both $P$. menglaensis and $P$. nuchalis. The nine evolutionary lineages revealed within clade A were distributed across two major clades, which we name herein: the $P$. carinatus species group (A1, lineages 1-6), and the $P$. nuchalis species group (A2, lineages 7-9; see Fig. 3):

a. Pareas carinatus species group (subclade A1; 1.0/100) comprised six lineages formerly recognized under the sole combination $P$. carinatus, including the populations from Peninsular Malaysia southwards from the Isthmus of Kra, corresponding to $P$. carinatus sensu stricto (1.0/100; lineage 5, see Fig. 3). Two samples from Tenasserim Mountains in Peninsular Thailand and Myanmar northwards from the Isthmus of Kra formed a monophyletic group (1.0/100; lineage 6, see Fig. 3), which represented the sister clade to the Malayan $P$. carinatus sensu stricto (1.0/100). The populations of $P$. carinatus from the mainland Indochina formed a monophyletic group (1.0/100), including three well-supported subgroups: (1) populations from lowlands of southern Vietnam, corresponding to the subspecies $P$. carinatus unicolor (Bourret, 1934) (1.0/100; lineage 1, see Fig. 3); (2) populations from the northern portion of Annamite (Truong Son) Mountains in central Vietnam and Laos (1.0/100; lineage 2, see Fig. 3); (3) populations from montane areas of Western Indochina (1.0/100; lineage 3, see Fig. 3), including the recently described $P$. menglaensis from 
497

498

499

500

501

502

503

504

505

506

507

508

509

510

511

512

513

514

515

516

517

518

519

520

521

522

523

524

525

526

527

southern Yunnan (locality 22, samples 39-43), and the topotypic specimen of $P$. berdmorei Theobald, 1868 from Mon State, Myanmar (locality 17, sample 45). Finally, a single specimen initially identified as $P$. cf. carinatus from Phu Yen Province in southern part of Central Vietnam formed a divergent lineage with sister relationships with all other populations of $P$. carinatus species group members from the mainland Indochina (1.0/94; lineage 4, see Fig. 3).

b. Pareas nuchalis species group (subclade A2; 0.99/80) got moderate node support level in the ML analysis since P. nuchalis from Borneo was only represented in our work by the single partial sequence of ND4 mtDNA gene (lineage 9, see Fig. 3). The two reciprocally monophyletic lineages from montane areas of Vietnam initially identified as $P$. cf. carinatus formed a wellsupported clade $(1.0 / 100)$ which is unexpectedly only distantly related to other mainland Southeast Asian members of Pareas and supposedly more closely related to $P$. nuchalis: the lineage from Kon Tum - Gia Lai Plateau in Central Annamites (1.0/100; lineage 7, see Fig. 3), and the lineage from Langbian Plateau in Southern Annamites, corresponding to the recently described $P$. temporalis (1.0/100; lineage 8, see Fig. 3).

Distribution of the phylogenetic lineages within the clade A is presented in Figure 1. Most lineages that cluster together in each of our two major subclades A1 and A2 are allopatrically distributed within the clade (Fig. 1). Two lineages from different subclades are found sympatrically: $P$. nuchalis (lineage 9) occurs in sympatry with $P$. carinatus sensu stricto (lineage 5 ) in Borneo and Sumatra, while $P$. cf. carinatus (lineage 1) occurs syntopically with lineage 8 of P. temporalis in Langbian Plateau of southern Vietnam (locality 37, Fig. 1). The only case of distribution overlap of lineages belonging to the same species group includes the lineages 3 and 6 of $P$. carinatus which are occur sympatrically in Suanphueng area of Ratchaburi Province in western Thailand (locality 14, Fig. 1). However in Suanphueng the co-occurring lineages of Pareas have clearly different habitat preferences and are not syntopically distributed: vouchers of lineage 3 were recorded in lowland bamboo forest at $300 \mathrm{~m}$ asl., while the voucher of lineage 6 was collected in the montane forest at ca. $800-1000 \mathrm{~m}$ asl.

\section{Sequence divergence}

Peer] reviewing PDF | (2021:08:64561:2:0:NEW 8 Dec 2021) 
The interspecific uncorrected genetic $p$-distances in cyt $b$ and ND4 mtDNA genes within 529 the genus Pareas are summarized in Supplementary Tables S7 and S8, respectively. For cyt $b$ 530 gene genetic divergence varied from $p=4.1 \%$ (between $P$. geminatus sensu stricto and $P$. 531 xuelinensis) to $p=25.2 \%$ (between $P$. kaduri and lineage 7 of $P$. cf. carinatus from Central 532 Annamites) (Supplementary Table S7). For ND4 gene $p$-distances varied from $p=5.2 \%$ (between 533 lineages 1 and 2 of the $P$. carinatus complex) to $p=23.7 \%$ (between $P$. nuchalis and P. komaii) 534 (Supplementary Table S8). In several cases the intraspecific distances within Pareas species 535 were greater than the minimal interspecific divergence values, which is likely explained with the 536 incompletely known taxonomy of the group: lineage 6 of the $P$. carinatus complex from 537 Tenasserim (5.0/4.2, hereafter values correspond to intraspecific distances for cyt $b / N D 4$ genes), 538 P. geminatus sensu lato (7.2/-), P. macularius (11.5/10.4), P. margaritophorus (5.2/4.9), and $P$. 539 monticola (3.7/5.7).

540

541 Divergence times estimation

542 The time-calibrated BEAST analysis recovered a phylogeny with well-supported nodes 543 (BPP $\geq 90$ ) throughout the tree, topologically identical to the BI tree (Fig. 2; Supplementary Fig. 544 S1). The phylogeny indicates that the most recent common ancestor (MRCA) of Pareinae 545 originated in late Eocene (Fig. 2). Basal radiation of Pareinae likely happened during the late 546 Eocene at approximately 39.3 mya, the group continued to radiate across Asia up until the

547 Pleistocene (Fig. 2). Diversification of the genera Asthenodipsas and Pareas started during the 548 early Oligocene (30.0 mya and 31.3 mya, respectively). The major lineages (i.e. species groups) 549 within the genus Pareas diversified between approximately 24.0-12.4 mya with species-level 550 radiations evolving up until 5.0-2.0 mya (Fig. 2). Estimated node-ages and the 95\% highest 551 posterior density (95\% HPD) for the main nodes are summarized in detail in Supplementary 552 Table S9.

553

554

\section{Biogeography}

All the trees generated in RASP analyses generally recovered the same ancestral range for 556 each node, thus converging on the same biogeographical scenario (Fig. 2). Model comparisons

557 showed that the Langrange Dispersal-Extinction-Cladogenesis (DEC) model is the best fit to the 558 data and most likely to infer the correct ancestral range at each node being the it had the highest 
559 and lowest log likelihood and AIC scores, respectively. Our analyses unambiguously suggested 560 that the MRCA of Pareinae (node 3; Supplementary Fig. S1; Fig. 2) most likely inhabited 561 Sundaland, which is also reconstructed as an ancestral range for the genera Asthenodipsas and 562 Aplopeltura (nodes 4 and 9, respectively; Supplementary Fig. S1; Fig. 2). The split between 563 Pareas and Aplopeltura is likely explained by a vicariant event between Sundaland and West 564 Indochina (Fig. 2). The divergence between the two major clades within the genus Pareas 565 coincides with a vicariance between Indo-Burma and Eastern Himalaya (ancestral range for clade 566 A) and West Indochina (ancestral range for clade B) (Fig. 2). Major ancestral nodes within the 567 Pareas clade A remained within Indo-Burma and Eastern Himalaya, from where its members at 568 least three times widely dispersed to the mainland East Asia and further southwards to Indochina 569 and independently twice eastwards to Taiwan and the Ryukyus (Fig. 2). Pareas clade B 570 expanded its range to East Indochina and at least twice dispersed to Sundaland (see Fig. 2). 571 Overall, our analysis suggests an "upstream" colonization hypothesis for the Pareinae (from 572 island to continent; see Filardi \& Moyle, 2005; Jønsson et al., 2011), and, thus, the subfamily 573 appears to have originated in Sundaland and then colonized the mainland Asia.

574

\section{Morphological differentiation}

576 The PCA of the morphological dataset on P. carinatus - P. nuchalis complex revealed that the most distant morphospatial separation occurs in P. nuchalis (lineage 9), P. temporalis 578 (lineage 8), P. cf. carinatus lineages from Kon Tum - Gia Lai Plateau (lineage 7), from Phu Yen 579 Province (lineage 4), and from Tenasserim (lineage 6); followed with general separation of the $P$. cf. carinatus lineage from northern Annamites (lineage 2) and cluster consisting of the lineages of $P$. carinatus sensu stricto from Sundaland (lineage 5), and $P$. cf. carinatus from western Indochina and Yunnan (lineage 3) and southern Vietnam (lineage 1) (Fig. 4). PC1 accounted for $18.5 \%$ of the variation in the data set and loaded most heavily for relative tail length, number of subcaudal scales, number of ventral scales, and number of prefrontals bordering eye (TaL/TL,

585 SC, VEN, and Prefr-eye; Supplementary Table S10). PC2 accounted for 14.8\% of the variation 586 in the data set and loaded most heavily for number of keeled dorsal scale rows, total length, head length, and tail length (KMD, TL, HL, and TaL; Supplementary Table S10). The univariate and multivariate morphological analyses further supported results of the molecular analyses by 
590 each other in morphospace and bear a number of statistically significant mean differences in

591 varying combinations of meristic and color pattern characters, thus providing reliable diagnostic 592 character differences among the species (Table 2; Supplementary Tables S11-13).

593

594

Systematics

595

596

597

\section{Genus-level taxonomy of Pareinae}

598

All recent phylogenetic studies on caenophidian snakes agree on the monophyly of Pareidae (Pyron, Burbrink \& Wiens, 2013; Figueroa et al., 2016; Zaher et al., 2019) and of the 599 subfamily Pareinae with respect to Xylophiinae (Deepak et al., 2019). Most works on

600

601

602

603

604

605

606

607

608

609

610

611

612

613

614

615

616

617

618

619

620 phylogenetic relationships of this group agreed that the genus Apolpeltura is a sister taxon of Pareas sensu lato, however, the monophyly of the genera Pareas and Asthenodipsas has been questioned for a long time.

Several earlier studies demonstrated that the P. carinatus complex (including P. nuchalis) is phylogenetically distant from other members of the genus, which was recovered as paraphyletic (e.g., Guo et al., 2011; Pyron et al., 2011). It was noted that these genetic divergence are concordant with differences in a number of external morphology and scalation characters (Guo et al., 2011) and scale ultrastructure (He, 2009; Guo et al., 2020) (see Fig. 5). Guo et al. $(2011,2020)$ suggested that $P$. carinatus and $P$. nuchalis are different from other Pareas species in morphological, ultrastructural and molecular characteristics, and therefore, they "might be removed from the genus Pareas"; this idea was further supported by Wang et al. (2020). However, due to incomplete sampling and insufficient morphological data, Guo et al. (2011, 2020) and Wang et al. (2020) refrained from making a formal taxonomic decision on the division of Pareas [Note - Wang et al. (2020) applied a new genus name 'Northpareas' to the clade including all Pareas species except P. carinatus, P. nuchalis, and P. menglaensis, however this name is only used in Appendix S3 of their paper and is not used in the text of their manuscript, and thus should be considered a nomen nudum]. At the same time, both Guo et al. (2011, 2020) and Wang et al. (2020) have overlooked two taxonomic issues:

1) Pareas carinatus Wagler, 1830 is the type species of the genus Pareas Wagler, 1830, and hence it cannot be placed to a different genus; in our analyses the name Pareas Wagler, 1830 corresponds to the Pareas clade A (see Fig. 3). 
621

622

623

624

625

626

627

628

629

630

631

632

633

634

635

636

637

638

639

640

641

642

643

644

645

646

647

648

649

650

651

2) Eberhardtia Angel, 1920 is an available genus-level name, erected for Eberhardtia tonkinensis Angel, 1920, which was considered a junior synonym of Pareas formosensis (Van Denburgh, 1909) by Ding et al. (2020); in our analyses it corresponds to the Pareas clade B (see Fig. 3).

Our phylogenetic analyses confirmed the existence of two highly divergent reciprocally monophyletic clades within Pareas sensu lato, while strongly supporting the monophyly of the genus.

The genus Asthenodipsas Peters, 1864 (type species - Asthenodipsas malaccana Peters, 1864) was for the long time considered a junior synonym of Pareas. Rao \& Yang (1992) examined morphological differences and placed the two species laevis Boie, 1827, and malaccana Peters, 1864 (that time members of the genus Pareas) to a newly erected genus Internatus Rao \& Yang, 1992 (type species - Amblycephalus laevis Boie, 1827). Further studies have also added the taxa tropidonota van Lidth de Jeude, 1923 and vertebralis Boulenger, 1900 to this genus (David \& Vogel, 1996; Grossmann \& Tillack, 2003). However, Iskandar \& Colijn ("2001" 2002) noted that Rao \& Yang (1992) had clearly overlooked an available name for the taxa assigned to their new genus Internatus, i.e., Asthenodipsas (Grossmann \& Tillack, 2003). A number of recent works described five additional species within the genus Asthenodipsas, without addressing questions of genus-level taxonomy of the group (Loredo et al., 2013; Quah et al., 2019, 2020, 2021). Several studies demonstrated that Asthenodipsas includes two major highly divergent clades, one including A. laevis and A. borneensis (belonging to the $A$. malaccana species complex) (clade $\mathrm{E}$ in our analyses, see Fig. 3), and another including $A$. vertebralis, A. tropidonota, and A. lasgalenensis (clade D in our analyses, see Fig. 3). Hence, both names Asthenodipsas and Internatus are referred to the members of clade E, while clade D has no available genus-level name. In previous phylogenetic studies, monophyly of the genus Asthenodipsas sensu lato was not supported (Guo et al., 2011) or got only moderate level of node support (Wang et al., 2020; Ding et al., 2020; Vogel et al., 2021). In our analyses, monophyly of Asthenodipsas sensu lato was strongly supported in the concatenated analysis of mtDNA + nuDNA data (Fig. 3), while the analysis of mtDNA genes alone suggested paraphyly of the genus with respect to Pareas + Aplopeltura (Supplementary Fig. S2). This genetic divergence among two clades of Asthenodipsas is concordant with significant differences in taxonomically valuable scalation characters, such as the number of chin shields and the number of infralabials in contact 
652 (see Fig. 5).

653 In summary, in the molecular phylogenetic analysis, Pareinae is obviously divided into five 654 major branches: (A) Pareas carinatus + P. nuchalis complex, (B) other species of Pareas, (C) 655 Aplopeltura, (D) Asthenodipsas vertebralis group, and (E) other species of Asthenodipsas (Fig. 656 3). Monophyly of both Pareas sensu lato (clades A + B) and Asthenodipsas sensu lato (clades $657 \mathrm{D}+\mathrm{E})$ is strongly supported, while Asthenodipsas does not seem to be monophyletic according to 658 mtDNA data alone. Should all five major lineages of Pareinae be recognized as distinct taxa?

659 As we argue below, we find there to be substantial evidence supporting the treatment of the 660 major clades within Pareas sensu lato and Asthenodipsas sensu lato as separate subgenera. The 661 taxonomic framework ideally should be optimized for utility, reflecting monophyly of taxa and 662 their differences in sets of biologically significant characters, as well as stability, reducing the 663 need for additional taxonomic changes in future (Vences et al., 2013; Gorin et al., 2021). 664 Although, the present evidence indicates that we can be confident in the respective monophyly of 665 Pareas sensu lato and of Asthenodipsas sensu lato, it should be noted that the basal radiations 666 within the both genera are very old: the two clades of Pareas diverged in early Oligocene (ca. 66731.3 mya), while the basal radiation within Asthenodipsas happened soon afterwards (ca. 30.0 668 mya). These estimates are comparable with the split between Pareas sensu lato and Aplopeltura 669 (ca. 33.6 mya), and are of equal or greater age than many other Caenophidian genera (see Zaher 670 et al., 2019). While taxon age is usually not taken into account in higher taxonomy, it is however 671 desirable for taxa of equal rank to be of generally comparable age (Hennig, 1966; Vences et al., 672 2013; Gorin et al., 2021). In addition to their substantial age, a number of important characters of 673 external morphology, scalation, and scale ultrastructure distinguish the major clades within 674 Pareas and Asthenodipsas, allowing their recognition both in collections and in the field 675 (summarized below in taxonomic accounts). Furthermore, there are pronounced differences in 676 the patterns of geographical distribution among the five clades of Pareinae: our hypothesis of the 677 biogeographic history of this subfamily demonstrated that while the whole group evolved in 678 Sundaland, Pareas clade A likely originated in Himalaya and Indo-Burma, and further dispersed 679 to East Asia and Indochina, while Pareas clade B likely originated in Western Indochina, from 680 where it colonized Sundaland and Eastern Indochina (Fig. 2). The cumulative evidence suggests 681 that the lack of taxonomic recognition for the major clades within the genera Pareas and 682 Asthenodipsas would conceal information on the ancient divergence between these lineages, as 
683 well as the significant differences between them in a set of biologically relevant traits 684 (summarized in Supplementary Table S14).

685 We propose to recognize the clades A and B of Pareas and clades D and E of 686 Asthenodipsas as separate subgenera. This would enhance the diagnosability of the respective 687 taxa and make them more comparable units to other genera of Pareinae, and as a consequence 688 fully stabilize the taxonomy of the subfamily. This taxonomic action would therefore be in 689 accordance with all three primary Taxon Naming Criteria (TNCs): Monophyly, Clade Stability, 690 and Diagnosability, as well as the secondary TNCs: Time Banding and Biogeography (see 691 Vences et al., 2013). The use of subgenera seems has been successfully applied in several recent 692 revisions of taxonomically challenging groups of reptiles, e.g. cobras of the genus Naja 693 (Wallach, Wuester \& Broadley, 2009), Trimeresurus, the Asian pit-vipers (David et al., 2011), 694 and Gekko (geckoes) (Wood et al., 2020). Wood et al. (2020) argued that the defining subgenera 695 may aide taxonomists in species descriptions by allowing them to only diagnose putatively new 696 species from the most relevant members of the same subgenus. By creating formally available 697 supraspecific taxa, accompanied by character-based diagnoses and properly assigned type 698 species, the practice of recognizing subgenera also has the potential to restrain taxonomic 699 vandalism, a malpractice forming a long-standing problem in systematics (Kaiser et al., 2013; 700 Wood et al., 2020; Wüster et al., 2021), and thus further enhance taxonomic stability.

701

702

\section{Species-level diversity in Pareinae}

Based on our updated phylogeny of Pareinae, we report on previously unrecognized 704 diversity within the subfamily, and also confirm several taxonomic conclusions made in earlier studies. We document the high degree of uncorrected pairwise sequence divergence between the two samples of Aplopeltura boa from Peninsular Malaysia and Sabah in Malaysian Borneo: with p-distances of $13.0 \%$ in cyt $b$ gene and $16.7 \%$ in ND4 gene, the divergence between these populations is estimated as 12.3 mya (Fig. 2; Supplementary Table S9). Further integrative taxonomic studies are needed to clarify the taxonomic status of Malayan and Bornean populations of $A$. boa that might lead to recognition of several species within the genus Aplopeltura.

In the present study we re-define species groups within the genus Pareas recognizing two 
714 species groups within the clade B (B1: P. hamptoni group; B2: P. chinensis group; B3: $P$. 715 margaritophorus group; and B4: P. monticola group) (Fig. 3). Within the clade B of Pareas our 716 results are largely concordant with a number of earlier studies. In P. montiocola group our 717 analysis further confirms the species status of the recently described P. victorianus (Vogel et al., 718 2021); the divergence between this species and its sister taxon P. monticola is estimated as 14.1 719 mya (Fig. 2). In P. margaritophorus group our results fully agree with the data of Vogel et al. 720 (2020) in recognizing $P$. andersonii, $P$. modestus, and $P$. macularius as species distinct from $P$. 721 margaritophorus. Moreover we report on a deep divergence between the two samples of $P$. 722 macularius from Myanmar and Laos with $p$-distances of $11.5 \%$ in cyt $b$ gene and $10.4 \%$ in ND4 723 gene (Supplementary Tables S7-S8); the divergence between these populations is estimated as 724 ca. 8.2 mya (Fig. 2), what might be an indicative of an incomplete taxonomy of the group. As the 725 type locality of P. macularius is located in Mon State, Myanmar, this raises the question of the 726 taxonomic status of the populations from Laos (this work is currently in progress and will be presented elsewhere). Within $P$. hamptoni species group we confirm the results of Ding et al. (2020) and Yang et al. (2021), suggesting that P. kaduri and P. nigriceps are sister taxa, while the phylogenetic position of $P$. vindumi remains unresolved. Genetic divergence among the three members of the Taiwan - Ryukyus clade of this group (P. atayal, P. komaii, and P. iwasakii) is comparatively low $(6.9 \%<p<9.0 \%$ in cyt $b$ gene; see Supplementary Table S7), and the basal radiation of this clade is estimated to happen only ca. 5.7 mya (Fig. 2). However a number of recent integrative studies, combining molecular, morphological, behavioral, and ecological data provide strong evidence that these taxa represent distinct species (You, Poyarkov \& Lin, 2015; Chang et al., 2021). Our data support the conclusions of Liu \& Rao (2021) which state that P. mengziensis, recently described by Wang et al. (2020) is conspecific to P. niger, a taxon which has been for a long time placed into a synonymy of $P$. hamptoni. Genetic divergence between $P$. niger and $P$. mengziensis is minimal ( $0.3 \%$ in cyt $b$ gene; see Supplementary Table S7), and given the morphological data reported by Liu \& Rao (2021) there is little doubt that the latter taxon represents a junior synonym of the former. We further confirm the earlier results of Ding et al. (2020) in assigning the majority of populations of $P$. hamptoni complex from Vietnam, including the specimen identified as "P. tonkinensis", to P. formosensis (Fig. 3). Our results also agree with that of Ding et al. (2020) in recognizing $P$. geminatus as a species distinct from $P$. 
745 data) are less clear. In our analysis, $P$. geminatus consists of two major lineages (P. geminatus 1

746 from northern Thailand and P. geminatus 2 from southern Yunnan of China and northern Laos),

747 and is paraphyletic with respect to $P$. xuelinensis, which is grouped with $P$. geminatus 1 with

748 strong support (Fig. 3). Two taxonomic solutions are possible to keep the monophyly of the

749 recognized taxa: (1) to split $P$. geminatus sensu lato and assign the Thai population to $P$.

750 xuelinensis; or (2) to consider P. xuelinensis a junior synonym of $P$. geminatus. Genetic

751 divergence among $P$. geminatus $1+P$. xuelinensis and $P$. geminatus 2 is low (4.1\% in cyt $b$

752 gene; see Supplementary Table S7), the divergence between these clades is estimated as only ca.

7532.0 mya (Fig. 2), while the morphological characters distinguishing $P$. xuelinensis from $P$.

754 geminatus are vague (see Supplementary Table S13). For the time being, we refrain from a

755 taxonomic decision on $P$. geminatus $-P$. xuelinensis clade pending further integrative studies to

756 address this problem, which should include additional materials from China and northern

757 Indochina.

758 Our study reports on a previously unrecognized diversity within the clade A of the genus

759 Pareas: altogether, we reveal nine well-supported and highly-divergent clades within this group,

760 five of which were previously unknown. Phylogenetic relationships among these lineages are

761 generally well resolved (Fig. 3) and genetic divergence between them varies from $p=4.8 \%$ to

$76222.1 \%$ in cyt $b$ gene, and from $p=5.2 \%$ to $20.1 \%$ in ND4 gene (Supplementary Tables S7-S8).

763 Recently Wang et al. (2020) revised the P. carinatus complex and described a new species from

764 southern Yunnan of China. In their analyses Wang et al. (2020) only included samples from

765 Peninsular Malaysia ( $P$. carinatus sensu stricto) and from Yunnan, and based on genetic

766 divergence and concordant morphological differences between these two populations, concluded

767 that the Yunnan population should be regarded as a new distinct species - P. menglaensis.

768 However, this taxonomic decision had several flaws:

769

770

771

772

773

774

775

1) Wang et al. (2020) only included in their analyses two populations of $P$. carinatus complex (from Yunnan and Peninsular Malaysia), but omitted any samples or sequences of $P$. carinatus complex from the major part of its range in Indochina and Sundaland, including the sequences available in GenBank.

2) Wang et al. (2020) overlooked two available species-level names presently coined as junior synonyms of $P$. carinatus: Pareas berdmorei Theobald, 1868, and Amblycephalus carinatus unicolor Bourret, 1934 (see Table 1). 
776

777

778

779

780

781

782

783

784

785

786

787

788

789

790

791

792

793

794

795

796

797

798

799

800

801

802

803

804

805

806

3) Finally, in their revision Wang et al. (2020) did not examine any type specimens of the P. carinatus species complex.

Our updated tree indicates that $P$. carinatus sensu lato is paraphyletic with respect to both P. menglaensis and P. nuchalis $+P$. temporalis, and that the taxonomy of the complex needs to be reconsidered. The preponderance of data suggests that the pronounced phylogeographic structure within P. carinatus - P. nuchalis groups that bear deep genetic divergences, generally wide morphospatial separation among the sampled populations, and statistically different character state means is indicative of a species complex and as such we consider each population to be recognized as a distinct taxon, which we formally describe below:

1) The lineage of $P$. cf. carinatus from mountains of central Vietnam (lineage 7) and $P$. temporalis from southern Vietnam (lineage 8) form a well-supported clade with sister relationships to $P$. nuchalis (though with moderate node support) (Fig. 3). Genetic distance between these lineages is high $(12.7 \%$ in cyt $b$ gene, $9.6 \%$ in ND4 gene; see Supplementary Tables S7-S8); the divergence between them is estimated as ca. 9.3 mya (Fig. 2); they differ in a number of taxonomically significant characters from each other and other congeners (see below; summarized in Table 2), and are widely separated in PCA analysis (Fig. 4). We recognize lineage 7 as a separate new species and together with $P$. nuchalis and $P$. temporalis assign them to the $P$. nuchalis species group (subclade A2, Fig. 3), while all other members of clade A we assign to the $P$. carinatus species group (subclade A1, Fig. 3).

2) The population from Peninsular Malaysia (lineage 5), which morphologically and biogeographically corresponds to $P$. carinatus sensu stricto, forms a clade with lineage 6 from Tenasserim Mountains in western Thailand and adjacent Myanmar. Lineages 5 and 6 are separated by the Isthmus of Kra and likely diverged ca. 5.0 mya (Fig. 2); they are characterized by a moderate level of divergence in mtDNA gene sequences $(p=$ $7.7 \%$ in cyt $b$ gene, $5.7 \%$ in ND4 gene; see Supplementary Tables S7-S8), well separated in PCA analysis (Fig. 4), and are diagnosed by stable differences in a number of morphological characters (see below). We propose to recognize the Tenasserim lineage 6 as a new subspecies within $P$. carinatus.

3) All samples of P. carinatus complex from the mainland Southeast Asia form a clade sister to the clade inhabiting Thai-Malay Peninsula and Sundaland. Within the mainland 
807

808

809

810

811

812

813

814

815

816

817

818

819

820

821

822

823

824

825

826

827

828

829

830

831

832

833

834

835

836

837

clade a single specimen from Phu Yen Province of Vietnam (lineage 4) is highly divergent $(p=11.9 \%-12.7 \%$ cyt $b$ gene, $13.8 \%-14.2 \%$ in ND4 gene; see Supplementary Tables S7-S8), forming a sister lineage with respect to all remaining populations (Fig. 3). Though no geographic barrier is known to separate the Phu Yen population from other mainland lineages of $P$. carinatus complex, the divergence between them is estimated as ca. 11.3 mya (Fig. 2). Moreover, the Phu Yen specimen is different from all other congeners in a number of diagnostic morphological features (see below) and is widely separated from them in the PCA morphospace (Fig. 4). Below we describe the Phu Yen lineage 4 as a new species.

4) Finally, all mainland populations of $P$. carinatus complex except the Phu Yen lineage 4 form a clade with three well-supported subclades (see Fig. 3): (1) the basal subclade (lineage 3) encompasses populations from northern Tenasserim to Thailand and Yunnan, and includes the topotypic population of P. berdmorei from Mon State, Myanmar (Fig. 1, loc. 16) and P. menglaensis (Fig. 1, loc. 22); (2) populations from southern Vietnam (lineage 1), including the type of Amblycephalus carinatus unicolor in Kampong Speu, Cambodia (Fig. 1, loc. 29); and (3) populations from Northern Annamites in Vietnam and Laos (lineage 2). These three lineages are separated from each other by moderate genetic distances $(p=4.8 \%-7.4 \%$ in cyt $b$ gene, $5.2 \%-6.8 \%$ in ND4 gene; see Supplementary Tables S7-S8) with estimated divergence times of 5.94.0 mya. They are only partially separated in PCA analysis, with a wide overlap in the morphospace for lineages 1 and 3, and moderate separation of lineage 2 (Fig. 4), but are readily distinguished from each other in a number of chromatic and certain morphological differences (see below). We thus suggest that $P$. menglaensis Wang et al., 2020 represents a subjective junior synonym of P. berdmorei Theobald, 1868, and propose to recognize $P$. berdmorei as a full species with three subspecies: berdmorei (for lineage 3), unicolor (for lineage 1), and a new subspecies for lineage 2 described below.

In the updated taxonomy for $P$. carinatus $-P$. nuchalis complex we propose to recognize two new species and two new subspecies (see above). Though there has been a certain skepticism regarding the usage of subspecies in herpetological taxonomy in the past (e.g., Wilson \& Brown, 1953; Frost \& Hillis, 1990; Frost, Kluge \& Hillis, 1992), recently the category of subspecies is 
838 getting more popular in scope of wide application of phylogenomic data allowing to reveal new

839

840

841

842

843

844

845

846

847

848

849

850

851

852

853

854

855

856

857

858

859

860

861

862

863

864

865

866

867

868 cases of mito-nuclear discordance due to ongoing or ancient hybridization (e.g., Kindler \& Fritz, 2018; De Queiroz, 2020; Hillis, 2021; Marshall et al., 2021). Marshall et al. (2021) define the subspecies as a geographically circumscribed lineage that may has been temporarily isolated in the past, but which has since merged over broad zones of intergradation that show no evidence of reproductive isolation. We tend to tentatively recognize the lineages $1-3$ within $P$. berdmorei, and the lineages 5-6 within P. carinatus as subspecies but not as full species due to the following reasons: (1) genetic distances among the lineages 1-3 within P. berdmorei, and the lineages 5-6 within $P$. carinatus are notably lower than between the 'good' species within the $P$. carinatus group (see above); (2) though lineages 5-6 within P. carinatus are well separated in the PCA analysis, lineages 1-3 within P. berdmorei are poorly separated in the PCA analysis (see Fig. 4) and are differentiated from each other primarily by chromatic traits; (3) the estimated time of divergence between the lineages 1-3 (ca. 5.9-4.0 mya), and the lineages 5-6 (ca. 5.0 mya) within $P$. carinatus is notably younger than the age of divergence of 'good' species within the $P$. carinatus group (ca. 17.2-9.3 mya) and is comparable with the age of basal divergence of other wide-ranged species of Pareas, e.g. P. margaritophorus (ca. 5.2 mya), P. formosensis (ca. 3.6 mya), and P. monticola (ca. 3.4 mya) (see Fig. 2); (4) lineages 2 and 6 are represented in our analyses by a limited sampling of two specimens for each lineage; this material may be not sufficient to fully assess the variation of diagnostic morphological characters; (5) last but not least, Amblycephalus carinatus unicolor was traditionally recognized as a subspecies of $P$. carinatus (Nguyen, Ho \& Nguyen, 2009), thus keeping this taxon along with its sister lineages 2 and 3 in the rank of subspecies would support the taxonomic stability. Further studies including examination of additional materials and localities are needed to test whether the lineages within $P$. carinatus and $P$. berdmorei have zones of intergradation or are reproductively isolated from each other.

\section{Taxonomic accounts}

Family Pareidae Romer, 1956

Subfamily Pareinae Romer, 1956

Type genus: Pareas Wagler, 1830, by original designation. 
870

871

872

873

874

875

876

877

878

879

880

881

882

883

884

885

886

887

888

889

890

891

892

893

894

895

896

897

898

899

\section{Revised key to the genera and subgenera of the subfamily Pareinae}

1a. Dorsal scales in 13 rows; subcaudals undivided......genus Aplopeltura

1b. Dorsal scales in 15 rows; all subcaudals divided......2

2a. Anterior single inframaxillary shield absent; vertebrals scales weakly or not enlarged; preocular and subocular scales present; supralabials usually not in contact with the eye......genus Pareas

2aa. Frontal hexagonal with its lateral sides parallel to the body axis; anterior pair of chin shields generally broader than long or slightly longer; two or three distinct narrow suboculars......subgenus Pareas

$2 a b$. Frontal subhexagonal with the lateral sides converging posteriorly; anterior pair of chin shields much longer than broad; one thin elongated subocular......subgenus Eberhardtia stat. nov.

2b. Anterior single inframaxillary shield present; vertebral scales strongly enlarged; preocular and subocular scales absent; supralabials in contact with the eye......genus Asthenodipsas

2ba. Two pairs of chin shields; the third pair of infralabials in contact with each other......subgenus Asthenodipsas

$2 b b$. Three pairs of chin shields; the first pair of infralabials in contact with each other...... subgenus Spondylodipsas subgen. nov.

Phylogenetic definition: Pareinae is a maximum crown-clade name referring to the clade originating with the most recent common ancestor of Asthenodipsas malaccana and Pareas carinatus, and includes all extant species that share a more recent common ancestor with these taxa than with Xylophis captaini.

Updated diagnosis: Body strongly laterally compressed with long tail; a short skull, head strongly distinct from neck; large eyes with vertical pupil; vertebrals sharp, weakly enlarged or not enlarged; 13-15 rows dorsal scale rows (DSR) throughout the body; no mental groove; ventrals preceded by a strongly enlarged preventral larger than the first ventral scale.

Natural history notes: Pareinae are nocturnal, generally arboreal, oviparous snakes, mainly inhabiting of moist tropical and subtropical forests, all members are specialized feeders 
900

901

902

903

904

905

906

907

908

909

910

911

912

913

914

915

916

917

918

919

920

921

922

923

924

925

926

927

928

929

930

on snails and slugs.

Distribution: Widely distributed through the Oriental zoogeographic region from Eastern Himalaya and Northeastern India, central, southern and eastern China including the islands of Hainan and Taiwan, the Yaeyama group of the Ryukyus across the Indochina and the ThaiMalay Peninsula to the Greater Sunda Islands.

Content: includes all members of the three genera: Pareas Wagler, 1830, Aplopeltura Duméril, 1853, and Asthenodipsas Peters, 1864 (see below).

English name: Slug-eating snakes or Snail-eating snakes.

Remark: Diagnostic morphological features for the genera and subgenera of the subfamily Pareinae recognized herein are summarized in Supplementary Table S14. Genus Asthenodipsas Peters, 1864

Type species: Asthenodipsas malaccana Peters, 1864: 273-274, pl., figs. 3, 3a-3d, by monotypy.

Synonyms: Internatus Yang \& Rao in Rao \& Yang, 1992 (type species - Amblycephalus laevis Boie, 1827).

Phylogenetic definition: Asthenodipsas sensu lato is a maximum crown-clade name referring to the clade originating with the most recent common ancestor of Asthenodipsas malaccana and Asthenodipsas vertebralis, and includes all extant species that share a more recent common ancestor with these taxa than with any of the type species of other Pareinae genera recognized herein.

Updated diagnosis: Dorsal scales smooth, in 15 rows throughout the body; vertebrals enlarged, hexagonal; sharp vertebral keel developed; head distinct from neck, snout blunt; one or two loreals; preocular and subocular scales absent; supraoculars may be fused to the postoculars; nasal undivided; prefrontal, loreal and at least one supralabial in contact with the eye; supraoculars may be fused to the postocular; frontal subhexagonal with the lateral sides converging posteriorly; two anterior temporals; the anterior single inframaxillary shield present (Fig. 5C-F); inframaxillaries wider than long in two or three pairs; the first or third pair of inframaxillaries in contact with each other (Fig. 5C-F); cloacal plate entire; subcaudals divided (Peters, 1864; Grossmann \& Tillack, 2003; Quah et al., 2019, 2020; our data; see Supplementary Table S14).

Peer] reviewing PDF | (2021:08:64561:2:0:NEW 8 Dec 2021) 
931

932

933

934

935

936

937

938

939

940

941

942

943

944

945

946

947

948

949

950

951

952

953

954

955

956

957

958

959

960

961

Distribution: Sundaic region, including the southern Peninsular Thailand, West Malaysia, and the Greater Sunda Islands (Sumatra, Java and Borneo).

Content: Nine species, including A. borneensis Quah, Grismer, Lim, Anuar \& Chan; $A$. ingeri Quah, Lim \& Grismer, 2021; A. jamilinaisi Quah, Grismer, Lim, Anuar \& Imbun; $A$. laevis (Boie); A. malaccana Peters; A. lasgalenensis Loredo, Wood, Quah, Anuar, Greer, Ahmad \& Grismer; A. tropidonota (Lidth de Jeude); A. stuebingi Quah, Grismer, Lim, Anuar \& Imbun; and $A$. vertebralis (Boulenger).

Etymology: The genus name is derived from the Greek word "asthenos" ( $\alpha \sigma \theta \varepsilon v \omega$ s) for "weak", "lacking strength", and the generic name "Dipsas", which is believed to come from the name of a snake in Greek mythology "Dipsas" ( $\triangle \imath \psi \alpha \varsigma)$, the bite of which was believed to cause

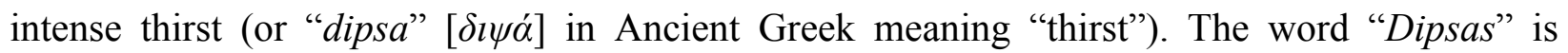
feminine in gender, therefore Asthenodipsas must be treated as feminine, and the names of the included species have to be adjusted to feminine gender (e.g., malaccana). The species name "tropidonotus" (meaning "keel-backed" in Greek) represents a latinized adjective and therefore its gender has to be adjusted to the feminine gender of Asthenodipsas as "tropidonota".

Recommended English name: Sundaic slug-eating snakes.

Material examined $(\mathbf{n}=\mathbf{3 8})$ : For the detailed information (specimen IDs, locality, sex, and the main morphological characteristics) of Asthenodipsas borneensis $(\mathrm{n}=1)$, A. laevis $(\mathrm{n}=15)$, A. lasgalensis $(n=5)$, A. malaccana $(\mathrm{n}=10)$, A. stuebingi $(\mathrm{n}=1)$, A. tropidonota $(\mathrm{n}=5)$, and $A$. vertebralis $(\mathrm{n}=1)$; see Supplementary Table S15 and Appendix II.

Remark: We recognize the following two subgenera within the genus Asthenodipsas for the $A$. laevis and $A$. vertebralis species groups based on stable morphological differences between their members concordant with the ancient phylogenetic divergence between these groups (see above). We propose the subgenus level for the taxa recognized below based on (1) strong support for the monophyly of the genus Asthenodipsas sensu lato (see above); (2) biogeographic similarity between these two groups, which are both distributed in the Sundaland region. 
Synonyms: Internatus Yang \& Rao in Rao \& Yang, 1992 (type species - Amblycephalus 963 laevis Boie, 1827).

964 Phylogenetic definition: Asthenodipsas sensu stricto is a maximum crown-clade name 965 referring to the clade originating with the most recent common ancestor of Asthenodipsas 966 malaccana and A. laevis, and includes all extant species that share a more recent common 967 ancestor with these taxa than with $A$. vertebralis.

968 Diagnosis: The subgenus Asthenodipsas differs from the subgenus Spondylodipsas 969 subgen. nov. (described below) by the following morphological characters: two pairs of chin 970 shields (vs. three pairs); 5-7 supralabials; 4-7 infralabials; the third pair of infralabials in contact 971 with each other behind the single anterior inframaxillary shield (vs. the first pair) (Fig. 5C-D; see 972 Supplementary Table S14 for details).

973 Distribution: Southern Peninsular Thailand, West Malaysia, Borneo, Sumatra, Java, and 974 Bangka islands, Mentawai, and Natuna Archipelagos.

975 Content: Six species, including A. borneensis Quah, Grismer, Lim, Anuar \& Chan; $A$. 976 ingeri Quah, Lim \& Grismer; A. jamilinaisi Quah, Grismer, Lim, Anuar \& Imbun; A. laevis 977 (Boie); A. malaccana Peters; and A. stuebingi Quah, Grismer, Lim, Anuar \& Imbun.

978 Recommended English name and Etymology: as for the genus Asthenodipsas. 979 designation.

Subgenus Spondylodipsas Poyarkov, Nguyen TV \& Vogel subgen. nov. [urn:1sid:zoobank.org:act:3FE7563C-2BFE-4BA4-A084-1A66E3D9B706]

Phylogenetic definition: Spondylodipsas subgen. nov. is a maximum crown-clade name referring to the clade originating with the most recent common ancestor of Asthenodipsas vertebralis and A. lasgalenensis, and includes all extant species that share a more recent common ancestor with these taxa than with $A$. malaccana.

Diagnosis: The subgenus Spondylodipsas subgen. nov. differs from Asthenodipsas sensu stricto by the following characteristics: three pairs of chin shields (vs. two pairs); 6-8 supralabials; 6-8 infralabials; the first pair of infralabials in contact with each other behind the mental (vs. the third pair) (Fig. 5E-F; see Supplementary Table S14 for details).

Distribution: Mountain areas of Sumatra and Peninsular Malaysia, and Pulau Tioman. 
Content: Three species, including A. lasgalenensis Loredo, Wood, Quah, Anuar, Greer,

994

995

996

997

998

999

1000

1001

1002

1003

1004

1005

1006

1007

1008

1009

1010

1011

1012

1013

1014

1015

1016

1017

1018

1019

1020

1021

1022

1023

Ahmad \& Grismer, A. tropidonota (Lidth de Jeude), and A. vertebralis (Boulenger).

Etymology: The genus name is a Latinized noun in feminine gender and is derived from the Greek word "spondylon" ( $\sigma \pi o v \delta v ́ \lambda \omega v)$ for "vertebra", and the generic name "Dipsas" (for etymology of this name see above). The name is given in reference to the well-developed vertebral keel in the members of the subgenus.

Recommended English name: Ridged slug-eating snakes.

\section{Genus Aplopeltura Duméril, 1853}

Type species: Amblycephalus boa Boie, 1828: 1035, by monotypy.

Phylogenetic definition: Aplopeltura is a maximum crown-clade name referring to Aplopeltura boa originating as the sister lineage to Pareas sensu lato.

Updated diagnosis: Dorsal scales smooth, in 13 rows throughout the body; vertebral keel weakly developed; two or three loreals; preocular and subocular scales present; supralabials not in contact with the eye; three anterior temporals; the anterior single inframaxillary shield absent (Fig. 5G); generally four (rarely three) pairs of chin shields, anterior pair of chin shields broader than long; at least the first and second pairs of chin shields in contact; subcaudals undivided (Duméril, 1853; Taylor, 1965; our data; see Supplementary Table S14 for details).

Distribution: Sundaic region, including: southern Peninsular Thailand, Peninsular Malaysia, Borneo, Sumatra, Java, Nias, Bangka, and Natuna Islands, and the Philippines (reliably recorded from the Balabac, Basilan, Mindanao, Palawan, and Luzon islands). The published record from southern Peninsular Myanmar by Dowling \& Jenner (1998) requires further verification.

Content: A monotypic genus including the single species, A. boa Boie.

Etymology: The genus name is likely derived from the Greek words "aplos" ( $\alpha \pi \lambda \omega ́ \varsigma)$ for "simple", and "pelte" ( $\pi \dot{\lambda} \lambda \tau \eta)$, for "scale", originally a name of a type of a small shield used in Ancient Greece.

Recommended English name: Blunt-headed slug-eating snakes.

Material examined $(\mathbf{n}=\mathbf{2})$ : For detailed information (specimen IDs, locality, sex, and main morphological characteristics) of Aplopeltura boa $(\mathrm{n}=2)$ see Supplementary Table S15 and Appendix II.

Peer) reviewing PDF | (2021:08:64561:2:0:NEW 8 Dec 2021) 
Remark: Our study reports on the significant genetic divergence between the samples of

1025

1026

1027

1028

1029

1030

1031

1032

1033

1034

1035

1036

1037

1038

1039

1040

1041

1042

1043

1044

1045

1046

1047

1048

1049

1050

1051

1052

1053

1054

A. boa from Borneo (Sabah) and Peninsular Malaysia, corresponding to the species level in Pareinae (see above). Further integrative taxonomic studies are required to clarify the status of the lineages within this species.

\section{Genus Pareas Wagler, 1830}

Type species: Dipsas carinata Wagler, 1830, by monotypy (see account for Pareas carinatus for details).

Phylogenetic definition: Pareas sensu lato is a maximum crown-clade name referring to the clade originating with the most recent common ancestor of Pareas carinatus and $P$. formosensis, and includes all extant species that share a more recent common ancestor with these taxa than with any of the type species of other Pareinae genera recognized herein.

Updated diagnosis: Dorsal scales smooth or keeled, in 15 rows throughout the body; vertebrals slightly larger than other dorsal scales or not enlarged; one (rarely two) loreals; preocular and subocular scales present; supralabials generally not contacting the eye (except for P. monticola and P. stanleyi); three anterior temporals; the anterior single inframaxillary shield absent (Fig. 5H-G); three pairs of inframaxillaries, all in contact with each other; subcaudals divided (Wagler, 1830; Smith, 1943; Taylor, 1965; Vogel et al., 2020; our data; see Supplementary Table S14 for details).

Distribution: Widely distributed throughout the Oriental zoogeographic region from Northeastern India and Himalaya to Eastern China and the Greater Sunda Islands.

Content: 26 species, including P. andersonii Boulenger; P. atayal You, Poyarkov \& Lin; P. berdmorei Theobald; P. boulengeri (Angel); P. carinatus Wagler; P. chinensis (Barbour); P. formosensis (van Denburgh); P. geminatus Ding, Chen, Suwannapoom, Nguyen, Poyarkov \& Vogel; P. hamptoni (Boulenger); P. iwasakii (Maki); P. kaduri Bhosale, Phansalkar, Sawant, Gowande, Patel \& Mirza; P. komaii (Maki); P. macularius Theobald; P. margaritophorus (Jan); P. modestus Theobald; P. monticola (Cantor); P. niger (Pope); P. nigriceps Guo \& Deng; P. nuchalis (Boulenger); P. stanleyi (Boulenger); P. temporalis Le, Tran, Hoang \& Stuart; P. victorianus Vogel, Nguyen \& Poyarkov; P. vindumi Vogel; P. xuelinensis Liu \& Rao; and the two new species described herein below: P. abros Poyarkov, Nguyen \& Vogel sp. nov.; and $P$. kuznetsovorum Poyarkov, Yushchenko \& Nguyen sp. nov. 
Etymology: The genus name is a latinized noun in masculine gender derived from the

1056

1057

1058

1059

1060

1061

1062

1063

1064

1065

1066

1067

1068

1069

1070

1071

1072

1073

1074

1075

1076

1077

1078

1079

1080

1081

1082

1083

1084

1085

Greek noun "pareias" ( $\pi \alpha \rho \varepsilon i \alpha \varsigma)$, a name of a mythological snake dedicated to Asclepius, and which was believed to be non-venomous and create a furrow anytime it moves.

Recommended English name: Oriental slug-eating snakes.

Material examined ( $\mathbf{n}=\mathbf{2 6 5})$ : Detailed information (specimen IDs, locality, sex, and main morphological characteristics) for P. abros $(\mathrm{n}=3), P$. berdmorei $(\mathrm{n}=21), P$. carinatus $(\mathrm{n}=26), P$. kuznetsovorum $(\mathrm{n}=1), P$. nuchalis $(\mathrm{n}=9)$, and P. temporalis $(\mathrm{n}=6)$ is presented in Table 2 and Appendix II; for P. vindumi $(\mathrm{n}=1)$ see Vogel (2015); for P. andersonii $(\mathrm{n}=13)$, P. macularius ( $\mathrm{n}=15)$, P. margaritophorus $(\mathrm{n}=51)$, and P. modestus $(\mathrm{n}=8)$ see Vogel et al. (2020); for P. geminatus $(\mathrm{n}=9)$ and P. hamptoni $(\mathrm{n}=5)$ see Ding et al. (2020); for P. formosensis $(\mathrm{n}=29), P$. kaduri $(\mathrm{n}=1)$, P. monticola $(\mathrm{n}=24)$, and P. victorianus $(\mathrm{n}=1)$ see Vogel et al. (2021); for the abovementioned species and $P$. atayal $(\mathrm{n}=6), P$. boulengeri $(\mathrm{n}=10), P$. chinensis $(\mathrm{n}=7), P$. komaii $(\mathrm{n}=9), P$. stanleyi $(\mathrm{n}=4), P$. xuelinensis $(\mathrm{n}=3)$ and $P$. cf. yunnanensis $(\mathrm{n}=3)$ the information is summarized in Supplementary Table S16 and Appendix II.

Remark: The taxonomic status of Amblycephalus yunnanensis Vogt (1922), currently considered a junior synonym of Pareas chinensis, is unclear due to the high morphological similarity within the group and the geographic proximity of the type localities of two taxa (both described from Yunnan Province in China). Ding et al. (2020) discussed this issue and suggested that the integrative taxonomic analysis including detailed comparisons of the type specimens is required to clarify the relations of these taxa (see Supplementary Table S13). Several recent studies on phylogenetic relationships within Pareas have revealed a deep divergence within the group, suggesting that its taxonomy still may be incomplete (Guo et al., 2011; You, Poyarkov \& Lin, 2015; Wang et al., 2020; Bhosale et al., 2020; Ding et al., 2020; Vogel et al., 2020, 2021).

\section{Subgenus Pareas Wagler, 1830}

Type species: Dipsas carinata Wagler, 1830, by monotypy (see the account for Pareas carinatus for details).

Phylogenetic definition: Pareas sensu stricto is a maximum crown-clade name referring to the clade originating with the most recent common ancestor of Pareas carinatus and Pareas nuchalis, and includes all extant species that share a more recent common ancestor with these taxa than with Pareas formosensis. 
Diagnosis: The members of the subgenus Pareas differ from the members of the subgenus

1087

1088

1089

1090

1091

1092

1093

1094

1095

1096

1097

1098

1099

1100

1101

1102

1103

1104

1105

1106

1107

1108

1109

1110

1111

1112

1113

1114

1115

Eberhardtia (designated below) by the following morphological characteristics: frontal hexagonal with the lateral sides parallel to each other (Fig. 5B); anterior pair of chin shields broader than long (Fig. 5H); two or three distinct narrow suboculars; and the ravine-like ultrastructure of dorsal scales (Wagler, 1830; Smith, 1943; Taylor, 1965; Vogel et al., 2020; He, 2009; Guo et al., 2020; our data; see Supplementary Table S14 for details).

Distribution: Distributed in the south-eastern part of the Oriental zoogeographic region from the southernmost China throughout the Indochina Peninsula to Peninsular Malaysia, Sumatra, Java, and Borneo (see Fig. 1).

Content: Six species, including P. berdmorei Theobald (with three subspecies: $P$. $b$. berdmorei stat. nov., P. b. unicolor comb. nov., and $P$. b. truongsonicus ssp. nov.); $P$. carinatus Wagler (with two subspecies: P. c. carinatus, and P. c. tenasserimicus ssp. nov.); $P$. nuchalis (Boulenger); P. temporalis Le, Tran, Hoang \& Stuart, 2021; and the two new species described herein below: P. abros Poyarkov, Nguyen \& Vogel sp. nov.; and P. kuznetsovorum Poyarkov, Yushchenko \& Nguyen sp. nov.

Recommended English name and Etymology: as for the genus Pareas.

\section{Key to the species of the subgenus Pareas}

1a. Prefrontal contacting the eye.....P. nuchalis

1b. Prefrontal not contacting the eye.....2

2a. Ratio $\mathrm{TaL} / \mathrm{TL} \geq 0.25$; large black blotch or a ring-shaped pattern on the nuchal area......3

2b. Ratio $\mathrm{TaL} / \mathrm{TL}<0.25$; large black blotch or a ring-shaped figure on the nuchal area absent......5

3a. All dorsal scales smooth, $\mathrm{VEN}<170$; black blotch on the nuchal area not forming a ring-shaped pattern......P. kuznetsovorum sp. nov.

3b. At least some dorsal scales strongly or slightly keeled, VEN $>170$; black blotch on the nuchal area forming a ring-shaped pattern......4

4a. All dorsal scale rows strongly keeled; VEN $>185$; no transverse dark bands on the body....... temporalis 
1116

1117

1118

1119

1120

1121

1122

1123

1124

1125

1126

1127

1128

1129

1130

1131

1132

1133

1134

1135

1136

1137

1138

1139

1140

1141

1142

1143

1144

1145

1146

4b. 9-11 rows of dorsal scales keeled at midbody; VEN $<185$; faint transverse dark bands on the body......P. abros sp. nov.

5a. Total length medium (up to $702 \mathrm{~mm}$ ); dorsal scales generally keeled in 3-11 upper rows at midbody; upper postorbital stripes thick, contacting each other on the nape generally forming a X-or )(-shaped pattern; territories southwards from the Tenasserim Range in Thailand......P. carinatus

5aa. VEN $\leq 190$; $\mathrm{SC} \leq 90$; body with transverse dark bands; territories south of the Isthmus of Kra.....P. c. carinatus

$5 a b . \mathrm{VEN}>190 ; \mathrm{SC}>90$; uniform light brown coloration of dorsum lacking transverse dark bands; Tenassenrim Range northwards from the Isthmus of Kra....P. c. tenasserimicus ssp. nov.

5b. Total length large (up to $770 \mathrm{~mm}$ ); dorsal scales keeled in 3-13 upper rows at midbody; upper postorbital stripes thin, generally forming a Y-shaped pattern on the nape or absent; mainland Indochina north from the Isthmus of Kra.....P. berdmorei

5ba. VEN 166-186; SC 57-89; dorsal scales keeled in 5-13 rows at midbody; body with transverse dark bars; ventral surfaces immaculate; iris golden-bronze to orange; restricted to northern Vietnam, northern Laos, northern Thailand, eastern Myanmar (northern Tennasserin), and southern Yunnan......P. b. berdmorei stat. nov.

5bb. VEN 162-180; SC 57-75; dorsal scales keeled in 3-9 rows at midbody; body uniform orange to beige coloration lacking dark markings; ventral surfaces generally immaculate; iris bright orange-red; restricted to southern Vietnam and eastern Cambodia......P. b. unicolor comb. nov.

5bc. VEN 187; SC 66-73; dorsal scales keeled in 13 rows at midbody; dense brownish mottling and spots on dorsal, lateral, and ventral surfaces of the head and body; iris uniform off-white to golden; restricted to the northern Annanmites (Truong Son) Mountains in central Vietnam and Laos......P. b. truongsonicus ssp. nov.

\section{Pareas carinatus species group}

The monophyly of the carinatus group is well supported in both analyses (A1, Fig. 3); the members of this species group are widely distributed across the Indochina from southern Yunnan Province of China to the Thai-Malay Peninsula southwards to Sumatra, Java, Borneo and smaller 
1147 adjacent islands (Fig. 1). Our phylogeny indicated that the group is composed of three species-

1148 level lineages, which were further supported by morphological analysis. The first lineage inhabits 1149 Sundaland and the Thai-Malay Peninsula and corresponds to $P$. carinatus sensu stricto. It 1150 includes two subgroups divided by the Isthmus of Kra, an important biogeographic boundary ( $D e$ 1151 Bruyn et al., 2005). The populations from Sundaland and the Thai-Malay Peninsula south of Kra 1152 correspond to P. c. carinatus (lineage 5, Fig. 3), while the populations northwards of Kra 1153 inhabiting the southern part of Tenasserim Range in western Thailand and adjacent Myanmar, we 1154 assign to a new subspecies $P$. c. tenasserimcus ssp. nov. described below (lineage 6, Fig. 3). The 1155 second lineage which we identify as P. berdmorei inhabits the mainland Indochina and includes three subgroups which we treat as subspecies. The first subgroup (lineage 3, Fig. 3) is widely distributed from southern Myanmar, western and northern Thailand, to Yunnan Province, China, and corresponds to $P$. b. berdmorei stat. nov.). The second subgroup (lineage 1, Fig. 3) is restricted to southern Vietnam and southeastern Cambodia and represents the subspecies $P$. $b$. unicolor comb. nov. The third subgroup recorded from the northern part of the Annamite Range we assign to a new subspecies $P$. b. truongsonicus ssp. nov. described below (lineage 2, Fig. 3). Finally, the third species-level lineage of this group was recorded from the north-western foothills of the Langbian (Da Lat) Plateau in southern Vietnam (lineage 4, Fig. 3); we below describe it as a new species $P$. kuznetsovorum sp. nov. Morphological data on the $P$. carinatus group members is summarized in Table 2 and Supplementary Tables S11-13. All members of the carinatus group lack the characteristic large black ring-shaped blotch on the nape and lateral sides of the neck; in P. kuznetsovorum sp. nov. the black blotch on the nape is present, but it is not ring-shaped.

\section{Pareas carinatus Wagler, 1830} indication on the basis of the NHM and RMNH catalogues. None given in the original description. - Status. Species name, as published in the binomen Dipsas carinata Wagler, 1830,

1175 placed on the 'Official List of Specific Names in Zoology', as Name Nr 2452, after Opinion 963 1176 of I.C.Z.N. (1971: 44). 
1178

1179

1180

1181

1182

1183

1184

1185

1186

1187

1188

1189

1190

1191

1192

1193

1194

1195

1196

1197

1198

1199

1200

1201

1202

1203

1204

1205

1206

1207

1208

Amblycephalus carinatus H. Boie in Schlegel, 1826: 1035 (nomen nudum).

Amblycephalus carinatus Boie, 1828: 251 (nomen nudum).

Pareas carinata Wagler, 1830: 181; Duméril, Bibron \& Duméril, 1854: 439.

Dipsas carinata — Schlegel, 1837: 285; Nguyen \& Ho, 1996: 61.

Leptognathus carinatus — Jan, 1863.

Amblycephalus carinatus - De Rooij, 1917: 277; Smedley, 1931: 53; Kopstein, 1936; Deuve, 1961: 30 .

Pareas carinatus - Cochran, 1930; Smith, 1943: 121; Manthey \& Grossmann, 1997: 376; Cox et al., 1998: 78; Schmidt \& Kunz, 2005: 41; Wallach, Williams \& Boundi, 2014: 535.

Lectotype (designated herein) (Fig. 6A; Fig. 7): RMNH 954C, adult male, collected by C. G. C. Reinwardt from Java Island, Indonesia. We designate the RMNH 954C as the lectotype, since it is the best preserved specimen of the type series fully agreeing with the original description, thus fulfilling the requirements of the Art. 74.7 of the Code (ICZN, 1999).

Paralectotypes (designated herein): Two specimens, RMNH 954A and RMNH 954B, both adult males, with the same collection data as the lectotype.

Updated diagnosis: Pareas carinatus differs from all other members of the genus Pareas by the following combination of morphological characters: body slender, maximal total length of $702 \mathrm{~mm}$; frontal scale hexagonal with lateral sides parallel to the body axis; anterior pair of chin shields longer than broad; loreal and prefrontal not contacting the eye; 1-3 suboculars; usually one postocular; temporals $3+4$ or $3+3$; three median vertebral rows slightly enlarged; 7-9 infralabials; 15 dorsal scale rows, at midbody the five upper rows might be slightly keeled; 158194 ventrals; 54-96 subcaudals, all divided; dorsum yellow-brown with dark vertebral blotches and dark mottling, transverse dark bands on the body present or absent; upper postorbital stripes continue to nape forming one or two longitudinal black spots; iris bronze laterally, beige dorsally (Wagler, 1830; our data).

Material examined: We directly examined 26 specimens of $P$. carinatus sensu stricto from Malaysia, Indonesia and Thailand (Supplementary Table 11Table 1).

Description of the lectotype (RMNH 954C) (Fig. 6A, Fig. 7): Adult male, body slender and notably flattened laterally; head comparatively large, narrowly elongated, clearly distinct from thin neck, snout blunt; eyes large, pupil vertical.

Body size. SVL 373 mm; TaL $101 \mathrm{~mm}$; TL $474 \mathrm{~mm}$; TaL/TL: 0.213 
Body scalation. Dorsal scales in 15-15-15 rows, slightly keeled at midbody, and without

1210

1211

1212

1213

1214

1215

1216

1217

1218

1219

1220

1221

1222

1223

1224

1225

1226

1227

1228

1229

1230

1231

1232

1233

1234

1235

1236

1237

1238

1239 apical pits; three vertebral scale rows slightly enlarged; outermost dorsal scale row not enlarged; ventrals 170 (+ 1 preventral), without lateral keels; subcaudals 67; cloacal plate single.

Head scalation. Rostral not visible from above; one nasal; two internasals, much wider than long, narrowing and slightly curving back laterally (in dorsal view), anteriorly in contact with rostral, laterally in contact with nasal and loreal, posteriorly in contact with prefrontal, not contacting preocular; two large irregular pentagonal prefrontals, much larger than internasals and with a slightly diagonal suture between them, not contacting the eye; frontal scale hexagonal with the lateral sides parallel to the body axis, longer than wide, smaller than parietals; one preocular; two suboculars; one postocular, not fused with subocular; one loreal in contact with prefrontal, not touching the eye; 7/7 supralabials, $3^{\text {rd }}$ and $5^{\text {th }}$ SL touching the subocular, none of them reaching the eye, $7^{\text {th }}$ by far the largest, elongated; temporals $3+4 ; 9 / 9$ infralabials, the anterior most in contact with the opposite along the midline, bordering mental, anterior 5 pairs of infralabials bordering anterior chin shields; 3 pairs of chin shields interlaced, no mental groove under chin and throat; anterior chin shields relatively large, generally wider than long, followed by two pairs of chin shields much wider than long.

Coloration. After over 200 years in preservative, the dorsal and ventral surfaces of the head, brownish with some dark-brown dusted spots (Fig. 7). Head with two lateral dark-brown postorbital stripes: the lower one is an interrupted dark line starting from the posterior edge of the eye, going diagonally down onto the anterior part of the last supralabial; the upper postorbital stripe is a dark-brown line running from the postocular backwards to the dorsal scales on the neck, where it meets the similar line on the opposite side of the body forming a narrow X-shaped dark-brown marking on the nuchal area (Fig. 7A). Upper labials marked with some fine irregular brown speckling (Fig. 7C-D). Dorsal surface is nearly uniformly light brown with slightly visible dark cross bands; ventral surfaces yellowish with sparse brownish mottling forming the interrupted line along the midline, descending backwards. Coloration in life unknown.

Comparisons: Pareas carinatus differs from Pareas berdmorei (revalidated below) by the generally smaller body size $(494.3 \pm 73.3 \mathrm{~mm}$ vs. $554.9 \pm 73.3 \mathrm{~mm})$; by slightly lower number keeled dorsal scale rows (6.5 \pm 2.9 vs. $8.9 \pm 2.8)$; and by generally thicker upper postorbital stripe and more pronounced dark markings on the nape (vs. thinner postorbital stripe and less pronounced dark markings on the nape); P. carinatus differs from $P$. nuchalis by prefrontal not 
1240 contacting the eye (vs. in contact); by the absence of the large ring-shaped black blotch on the 1241 nape (vs. present); by lower number of ventrals (171.35 \pm 9.3 vs. 209.89 \pm 5.3 ); lower number of

1242

1243

1244

1245

1246

1247

1248

1249

1250

1251

1252

1253

1254

1255

1256

1257

1258

1259

1260

1261

1262

1263

1264

1265

1266

1267

1268

1269

1270 subcaudals (73.3 \pm 7.6 vs. 111.1 \pm 6.1 ); and by having keeled dorsal scales (vs. dorsal scales totally smooth). Morphological comparisons between all species of the subgenus Pareas are detailed in Table 2. Pareas carinatus can be distinguished from other species of Pareas belonging to subgenus Eberhardtia stat. nov. by having two or three distinct narrow suboculars (vs. one thin and elongated) and by having a hexagonal frontal with its lateral sides parallel to the body axis (vs. subhexagonal) (see Supplementary Tables S13-S14).

Distribution: Based on molecular and morphological data, we suggest that this species is restricted to the Greater Sunda Islands (Java, Borneo and Sumatra), Peninsular Malaysia and Thailand, including the Tenasserim Mountains in western Thailand and south-eastern Myanmar, the northernmost known locality is Yaephyu, Tanintharyi Region, Myanmar (Fig. 1).

Etymology: The species name "carinatus" is a Latin adjective in nominative singular, masculine gender, derived from "carina" for a "keel of a ship", and is given in reference to the keeled dorsal scales in this species.

Recommended English name: Keeled slug-eating snake.

Remark: Based on the concordant results of morphological and molecular analyses, we recognize two subspecies within $P$. carinatus: the populations south of the Isthmus of Kra correspond to the nominative subspecies $P$. c. carinatus, while the populations from the Tenasserim Mountains northwards from Kra we describe below as P. c. tenasserimcus ssp. nov. Although the morphological variation among the sampled specimens of $P$. c. carinatus is significant (Fig. 4; Supplementary Tables S11-S12), in molecular analyses this subspecies is only represented by specimens from Peninsular Malaysia. Further phylogenetic analyses of $P$. carinatus populations from Java, Sumatra and Borneo are required and might reveal new presently unknown lineages within this species.

\section{Pareas carinatus carinatus Wagler, 1830}

Figures 6A, 7, 9A-C; Supplementary Tables S11-S12.

Dipsas carinata Wagler, 1830: 181.

\section{Chresonymy:}

Amblycephalus carinatus carinatus - (in part) Mertens, 1930. 
Pareas carinatus carinatus — (in part) Haas, 1950; Chan-ard et al., 1999: 177; Nguyen,

1272 Ho \& Nguyen, 2009: 374.

Diagnosis: Pareas carinatus carinatus differs from Pareas carinatus tenasserimicus ssp.

1274

1275

1276

1277

1278

1279

1280

1281

1282

1283

1284

1285

1286

1287

1288

1289

1290

1291

1292

1293

1294

1295

1296

1297

1298

1299

1300

1301 nov. described below by the following combination of morphological characters: maximal total length of $608 \mathrm{~mm}$; anterior pair of chin shields wider than long; one postocular; temporals generally $3+4$ (rarely $3+3,2+3$ ); 15 dorsal scale rows slightly keeled in 3-11 scale rows at midbody; 158-190 ventrals; 54-84 subcaudals; dorsum light brown with distinct dark vertebral spots and generally 44-73 transverse dark brownish or blackish bands (Fig. 9A-C); upper postorbital stripes generally contacting each other on the nuchal area forming a narrow X-shaped dark marking with curved branches; ventral scales yellowish with sparse brownish mottling forming the interrupted line along the midline.

Variation: Measurements and scalation features of the subspecies $P$. c. carinatus $(\mathrm{n}=25)$ are presented in Supplementary Tables S11. There is a certain variation among the sexes observed in the body size and the number of ventral scales. Males are generally smaller (TL 337.0-571.0 mm, average 471.5 $\pm 59.5 \mathrm{~mm}, \mathrm{n}=15$ ) than females (TL 446.0-608.0 mm, average $511.0 \pm 56.8 \mathrm{~mm}, \mathrm{n}=8)$; males also have a generally lower number of ventrals than females (158183, average $167.3 \pm 6.2, \mathrm{n}=15$ in males vs. $162-190$ average $175.2 \pm 8.8, \mathrm{n}=10$ in females). In five specimens from from Java (ZMH R11546 and R11542), Sumatra (SMF 37825-37826) and Borneo (NMW 28131:1) keels on dorsal scales are hardly visible; it is unclear if this feature reflects the geographic variation, or it might arise from the poor preservation of the specimens. Other morphological features showed no significant variation among the specimens of the series examined. In our phylogenetic analysis, $P$. c. carinatus was represented only by specimens from Peninsular Malaysia; further integrative molecular and morphological studies are needed to assess the geographic variation among the populations of $P$. c. carinatus from Java, Sumatra, Borneo, and Peninsular Malaysia.

Distribution: Peninsular Thailand south of the Isthmus of Kra, Malaysia (Peninsular Malaysia, Sarawak and Sabah within Borneo), Brunei, and Indonesia (Kalimantan Province, Sumatra, Java, Lombok, and Bali Islands) (Fig. 1).

Recommended English name and Etymology: as for Pareas carinatus.

Pareas carinatus tenasserimicus Poyarkov, Nguyen TV, Vogel, Pawangkhanant, 
1302

1303

1304

1305

1306

1307

1308

1309

1310

1311

1312

1313

1314

1315

1316

1317

1318

1319

1320

1321

1322

1323

1324

1325

1326

1327

1328

1329

1330

1331

1332

\section{Yushchenko \& Suwannapoom ssp. nov.} [urn:1sid:zoobank.org:act:11F7F6BA-4733-41FB-8E2D-405DCA5743E5]

Figures 6B, 8, 9D-E; Supplementary Tables S11-S12.

\section{Chresonymy:}

Pareas carinatus - (in part) Mulcahy et al., 2018: 98.

Holotype (Fig. 6B, Fig. 8): ZMMU R-16800 (field number NAP-10160), adult male, collected by P. V. Yushchenko from mountain forest in Joot Chomwil area, Suan Phueng District, Ratchaburi Province, western Thailand (N 13.56346, E 99.19465, elevation 800 m asl.) on 16 July 2019.

Diagnosis: Pareas carinatus tenasserimicus ssp. nov. differs from the nominative subspecies by the following combination of morphological characteristics: total length $702 \mathrm{~mm}$; anterior pair of chin shields as long as broad; two postoculars; temporals $3+3 ; 15$ dorsal scale rows slightly keeled in 7 scale rows at midbody; 194 ventrals; 96 subcaudals; dorsum light brown to beige, 73 weak dark vertebral spots; transverse dark bands on the body absent (Fig. 9D-E); upper postorbital stripes not contacting each other on the nuchal area forming a weak X-shaped dark marking with curved branches; ventral scales beige with dense brownish mottling not forming the interrupted midventral line.

Description of the holotype: Adult male, specimen in a good state of preservation (Fig. 8), body slender and notably flattened laterally; head comparatively large, narrowly elongated, clearly distinct from the thin neck, snout blunt; eye rather large, pupil vertical and slightly elliptical.

Body size. SVL 524 mm; TaL 178 mm; TL 702 mm; TaL/TL: 0.254.

Body scalation. Dorsal scales in 15-15-15 rows, slightly keeled in 7 upper scale rows at midbody, without apical pits; vertebral scales (three median rows) slightly enlarged; outermost dorsal scale row not enlarged; ventrals 194 (+ 1 preventral), lacking lateral keels; subcaudals 96, paired; cloacal plate single.

Head scalation. Rostral not visible from above; nasal single; two internasals, much wider than long, narrowing and slightly curving back laterally (in dorsal view), anteriorly in contact with rostral, laterally in contact with nasal and loreal, posteriorly in contact with prefrontal, not contacting preocular; two large irregular pentagonal prefrontals, much larger than internasals and with a slightly diagonal suture between them, not contacting the eye; frontal scale hexagonal with 
1333 the lateral sides parallel to the body axis, longer than wide, of the same size as the parietals; two 1334 preoculars; two suboculars; two postoculars, not fused with suboculars; one loreal in contact with

1335

1336

1337

1338

1339

1340

1341

1342

1343

1344

1345

1346

1347

1348

1349

1350

1351

1352

1353

1354

1355

1356

1357

1358

1359

1360

1361

1362

1363 prefrontal, not touching the eye; $7 / 7$ supralabials, $3^{\text {rd }}$ and $5^{\text {th }}$ SL touching suboculars, none of them reaching the eye, $7^{\text {th }}$ subocular the largest, elongate; temporals $3+3 ; 9 / 9$ infralabials, the anterior most in contact with the opposite along the midline, bordering mental, anterior five pairs of infralabials bordering anterior chin shields, $3^{\text {rd }}$ pair of infralabials in contact with each other (Fig. 8F); unpaired inframaxillary shield absent; two pairs of chin interlaced shields contacting each other, no mental groove under chin and throat; anterior chin shields relatively large, as long as broad, the second pair of chin shields much broader than long.

Coloration. In life, the dorsal and ventral surfaces of the head are uniform light brown dorsally, yellowish-beige ventrally (Fig. 9E). Head with two lateral postorbital stripes: the lower one is a thin dark-brown line starting from the lower posterior edge of the eye onto the anterior part of the last supralabial; the upper one is a strong dark-brown line running from postocular backwards to the medial dorsal scale rows on the neck; upper postorbital stripes not contacting each other on the nuchal area forming a weak X-shaped dark marking with curved branches. Upper labials marked with numerous irregular dark-brown speckling continuing and getting denser on lateral and dorsal surfaces of the head; $5^{\text {th }}$ supralabial with a larger black spot. Dorsal surfaces with ca. 73 faint dark blotches along the vertebral keel; transverse dark bands on the body absent; ventral surfaces of body and tail yellowish cream with very sparse small black spots concentrating laterally, dark spots and speckles getting denser on the posterior portion of the belly. Iris yellowish-orange laterally and ventrally, light beige dorsally; pupil black. In preservative: After two years of storage in ethanol (Fig. 8) the general coloration pattern has not changed; light brown of the coloration of dorsum, head and eye has faded becoming grayishbrown; other features of coloration remain unchanged.

Variation: A single male specimen observed in Kaeng Krachan N.P., Phetchaburi Province, Thailand (specimen not collected) is overall similar to the holotype of the new subspecies, but demonstrates certain differences in color pattern, including more pronounced dark vertebral spots and a series of dark spots along the lower raw of dorsal scales (Fig. 9D), while the holotype has a more uniform coloration lacking dark markings on dorsum and body sides (Fig. 9E). Given the geographic proximity of the Kaeng Krachan N.P. (Fig. 1, a) to the type locality of the new subspecies, and morphological similarity, we tentatively identify the Kaeng

Peer) reviewing PDF | (2021:08:64561:2:0:NEW 8 Dec 2021) 
1364 Krachan population as $P$. c. cf. tenasserimicus ssp. nov.; its taxonomic status requires further 1365 verification using morphological examination and molecular data.

1366 Comparisons: Pareas carinatus tenasserimicus ssp. nov. differs from $P$. c. carinatus by 1367 its generally larger size (702 mm vs. 337-608 [485.2 \pm 59.7$] \mathrm{mm}$ ); a slightly higher number of 1368 ventrals (194 vs. 158-190 [170.4 18.2$]$ ); a higher number of subcaudals (96 vs. 54-84 1369 [68.1 1 7.2]); by two postoculars (vs. single postocular); by the $3^{\text {rd }}$ pair of infralabials in contact 1370 with each other (vs. not in contact); by a uniform light brown coloration of dorsum lacking 1371 transverse dark bands (vs. transverse dark bands present); and by upper postorbital stripes not 1372 contacting each other on the nuchal area forming a weak dark marking with curved branches (vs. usually contacting each other forming a dark X-shaped marking). For the detailed comparison of the two subspecies of $P$. carinatus see Supplementary Table S12.

Distribution: To date the new subspecies is reliably known from only three localities in the southern portion of Tenasserim Range: the type locality in Suan Phueng District, Ratchaburi Province and Kaeng Krachan N.P., Phetchaburi Province of Thailand (locality 14, Fig. 1), and from Yaephyu area in Tanintharyi Region of Myanmar (locality 15, Fig. 1).

Etymology: The new subspecies name "tenasserimicus" is a Latin toponymic adjective in nominative singular, adopting the masculine gender of the genus name Pareas, and is given in reference to the Tenasserim Mountain Range in western Thailand and southeastern Myanmar, where the new subspecies occurs.

Recommended English name: Tenasserim slug-eating snake.

Ecology notes: The new subspecies inhabits montane evergreen forests of the Tenasserim Range on elevations above 800 asl. This is a nocturnal snake, all three specimens were recorded at night while perching or crawling on the tree branches and bushes ca. 1-2 $\mathrm{m}$ above the ground. The diet of the new subspecies is not known in detail, though it likely consists of land snails or slugs. In Suan Phueng area (locality 14, Fig. 1), the new subspecies occurs in sympatry with $P . b$. berdmorei, but was not recorded in the same habitats: the new subspecies inhabits montane forests at ca. 800-1000 $\mathrm{m}$ asl., while the specimens of $P$. b. berdmorei were recorded in lowland bamboo forest at $300 \mathrm{~m}$ asl. Other co-occurring species of Pareas include P. margaritophorus.

\section{Pareas berdmorei Theobald, 1868}


1395

1396

1397

1398

1399

1400

1401

1402

1403

1404

1405

1406

1407

1408

1409

1410

1411

1412

1413

1414

1415

1416

1417

1418

1419

1420

1421

1422

1423

1424

1425

Pareas berdmorei Theobald, 1868b: 63.

\section{Chresonymy and synonymy:}

Amblycephalus carinatus unicolor Bourret, 1934.

Pareas carinatus — (in part) Smith, 1943; Taylor, 1965; Yang \& Rao, 2008; Nguyen, Ho \& Nguyen, 2009; Teynié \& David, 2010; Le et al., 2014; Wallach, Williams \& Boundi, 2014; Chanard et al., 2015; Pham \& Nguyen, 2019.

Pareas berdmorei - Das et al., 1998.

Pareas carinatus unicolor — (in part) Nguyen, Ho \& Nguyen, 2009.

Pareas menglaensis Wang, Che, Liu, Ki, Jin, Jiang, Shi \& Guo, 2020.

Lectotype (designated herein) (Fig. 6C; Fig. 10): ZSI 8022, adult male collected by T. M. Berdmore from "Tenasserim", corresponding to Mon Region in southeastern Myanmar according to Das et al. (1998) (locality 16, Fig. 1). The specimen ZSI 8022 is designated herein as the lectotype as it is the best preserved specimen of the type series, and clearly corresponds to the morphological characteristics mentioned in the original description of Pareas berdmorei Theobald, 1868b, thus fulfilling the requirements of the Art. 74.7 of the Code (ICZN, 1999), modified by Anonymous (2003; Declaration 44).

Paralectotype (designated herein): one specimen, ZSI 8021, adult male, with the same collection information as the lectotype.

Referred specimen: ZSI 8023, juvenile, with the same collection information as the lectotype.

Remark: Theobald (1868b) described Pareas berdmorei based on a series of five specimens collected from "Tenasserim" by Major T. M. Berdmore. The description of Pareas berdmorei is based on a series of two adults; the three smaller specimens, which Theobald (1868b) were considered to be juveniles, were almost simultaneously described as $P$. macularius (Theobald, 1868a: 54). Therefore Theobald (1868b) proposed the new name for the two larger specimens "to prevent confusion of synonyms" (Theobald, 1868b: 63). According to Das et al. (1998), the type series of P. macularius includes three specimens ZSI 8024-26, while the specimens catalogued under the name P. berdmorei bear the museum numbers ZSI 8021-23. Bhosale et al. (2020) noted that only the specimens ZSI 8021 and ZSI 8022 are adults, therefore they correspond to the syntypes of $P$. berdmorei (Z. Mirza, pers. comm.).

We have obtained morphological data and a photograph of ZSI 8022, which we designate 
1426 as the lectotype of $P$. berdmorei, as it is the best preserved specimen of the type series which 1427 fully agrees with the original description of P. berdmorei (Theobald, 1868b: 63). We designate

1428

1429

1430

1431

1432

1433

1434

1435

1436

1437

1438

1439

1440

1441

1442

1443

1444

1445

1446

1447

1448

1449

1450

1451

1452

1453

1454

1455

1456

ZSI 8021 as the paralectotype, while ZSI 8023, without nomenclatural status, is listed in the referred specimens. The designation of lectotype for P. berdmorei is necessary due to the historical confusion between the descriptions of $P$. macularius and $P$. berdmorei; thus fulfilling the requirements of the Art. 74.7 of the Code (ICZN, 1999).

Updated diagnosis: Pareas berdmorei differs from other members of the genus Pareas by the following combination of morphological characters: maximal total length of $770 \mathrm{~mm}$; frontal scale hexagonal with its lateral sides parallel to the body axis; the anterior pair of chin shields broader than long; loreal and prefrontal not contacting the eye; generally 1 or 2 preoculars; regularly 2 (rarely 1 or 3 ) suboculars; generally single postocular (rarely 0 or 2); temporals $3+4$ or 3+3; one to three median vertebral dorsal scale rows slightly enlarged; generally 8 (7-9) infralabials; 15 dorsal scale rows, of them 3-13 scale rows at midbody feebly keeled; 162-187 ventrals; 57-89 subcaudals, all divided; dorsum yellow-brown to orange, dark markings on dorsum variable; thin upper postorbital stripes continue to nape often forming elongated dark markings; iris uniform, color varies from beige to bright reddish-orange (Theobald, 1868b; Bourret, 1934; Taylor, 1965; Ziegler et al., 2006; Yang \& Rao, 2008; Le et al., 2014;Pham \& Nguyen, 2019; Wang et al., 2020; our data).

Material examined: In this study we used morphological data from 38 specimens of $P$. berdmorei, including the 21 specimens examined directly (among them the lectotype of Pareas berdmorei Theobald and the holotype of Amblycephalus carinatus unicolor Bourret) and the published data for 17 specimens formerly listed as "P. carinatus" (Taylor, 1965; Ziegler et al., 2006; Yang \& Rao, 2008; Le et al., 2014; and Pham \& Nguyen, 2019), and "P. menglaensis" (Wang et al., 2020) (Supplementary Table S11).

Description of the lectotype (ZSI 8022): Adult male, a well-preserved specimen, with coloration significantly faded due to the long time of preservation in ethanol (Fig. 10), body slender and notably flattened laterally; head comparatively large, narrowly elongated, slightly distinct from neck, snout blunt; eyes large.

Body size. SVL 490 mm; TaL 120 mm; TL 610 mm; TaL/TL: 0.197.

Body scalation. Dorsal scales in 15-15-15 rows, slightly keeled in 9 scale rows at midbody, lacking apical pits; vertebral scales in three median rows slightly enlarged; the

Peer) reviewing PDF | (2021:08:64561:2:0:NEW 8 Dec 2021) 
1457 outermost dorsal scale row not enlarged; ventrals $174(+1$ preventral), lacking lateral keels; 1458 subcaudals 64; cloacal plate single.

1459 Head scalation. Rostral not visible from above; single nasal; two internasals, much wider 1460 than long, narrowing and slightly curving back laterally (in dorsal view), anteriorly in contact 1461 with rostral, laterally in contact with nasal and loreal, posteriorly in contact with prefrontal, not 1462 contacting preocular; two large irregular pentagonal prefrontals, much larger than internasals and

1463

1464

1465

1466

1467

1468

1469

1470

1471

1472

1473

1474

1475

1476

1477

1478

1479

1480

1481

1482

1483

1484

1485

1486

1487 with a slightly diagonal suture between them, not in contact with eye; the frontal scale hexagonal with the lateral sides parallel to the body axis, longer than wide, smaller than parietals; two preoculars; one subocular; one postocular, not fused with subocular; one loreal in contact with prefrontal, not touching the eye; 7/7 supralabials, $3^{\text {rd }}$ and $5^{\text {th }}$ SL touching the subocular, none of them reaching the eye, $7^{\text {th }}$ SL the largest, elongate; temporals $3+4 ; 8 / 8$ infralabials, 3 pairs of chin shields interlaced, all notably broader than long, no mental groove under chin and throat; anterior chin shields relatively large.

Coloration. Due to preservation in ethanol for over 150 years, the colors of the holotype have significantly faded, the specimen is uniform light brownish-yellow (Fig. 10A); the present body pattern of the lectotype no longer retains the original characteristics, though the thin dark postorbital stripes are still discernable and faded to orange-brown (Fig. 10B). In the original description, Theobald (1868: 63) gives the following information on the type coloration: "color is uniform ochraceous, with obsolete traces of vertical bands down the body; two dark lines converge on the nape; $<\ldots>$ belly white", indicating that the specimen has already significantly faded at the moment of the original description.

Comparisons: Pareas berdmorei is distinguishable from $P$. carinatus by the generally larger body size $(554.2 \pm 76.5 \mathrm{~mm}$ vs. $494.3 \pm 73.3 \mathrm{~mm})$; by slightly higher number of ventrals (177.3 \pm 5.8 vs. $171.4 \pm 9.3)$; slightly higher number of keeled dorsal rows (9.0 \pm 2.8 vs. $6.1 \pm 3.3)$; by generally less pronounced dark markings in the nuchal area, thinner postorbital stripes and a more uniform coloration of the iris; from P. nuchalis by prefrontal not contacting the eye (vs. in contact); by the absence of the ring-shaped black blotch on the nape (vs. present); by lower number of ventrals (177.3 \pm 5.8 vs. 209.9 \pm 5.3$)$; lower number of subcaudals (71.2 \pm 7.5 vs. 111.1 \pm 6.1 ); and by the presence of keeled dorsal scale rows (vs. dorsals totally smooth). Morphological comparisons between all species of the subgenus Pareas are detailed in Table 2 and Supplementary Table S13. Pareas berdmorei can be distinguished from other species of 
1488 Pareas belonging to subgenus Eberhardtia stat. nov. by having two or three distinct narrow 1489 suboculars (vs. one thin and elongated) and by having a hexagonal frontal with its lateral sides 1490 parallel to the body axis (vs. subhexagonal) (Supplementary Tables S13-S14).

Distribution: Pareas berdmorei is widely distributed across the mainland Indochina north

1492

1493

1494

1495

1496

1497

1498

1499

1500

1501

1502

1503

1504

1505

1506

1507

1508

1509

1510

1511

1512

1513

1514

1515

1516

1517

1518 from the Kra Isthmus (Fig. 1). Its range consists of the three major groups of populations (Fig. 1): (1) populations from southeastern Myanmar, western and northern Thailand, northern Laos and the southernmost parts of Yunnan Province of China; (2) populations from eastern Laos and central Vietnam located along the Truong Son Range; (3) populations from southern Vietnam and the adjacent parts of eastern Cambodia. As this species occurs in lowland to submontane tropical forests, it is seemingly absent from seasonally dry lowlands of central Indochina and the lower part of the Mekong Valley (Fig. 1). However, the occurence of P. berdmorei is expected in the Cardamom Mountains of Cambodia and the montane areas of eastern Thailand; therefore further field survey efforts are required to clarify the real extent of $P$. berdmorei distribution.

Etymology: Theobald (1868) named his new species in honor of British naturalist Captain Thomas Matthew Berdmore (1811-1859), who was the collector of the type specimens.

Recommended English name: Berdmore's slug-eating snake.

Remark: The cumulative evidence from molecular and morphological data strongly suggests that the populations of " $P$. carinatus" from the mainland Indochina are divergent and morphologically different from $P$. carinatus sensu stricto from Malayan Peninsula and the Greater Sunda Islands. Our results thus agree with the data of Wang et al. (2020), who compared the samples of $P$. carinatus group from southern Yunnan Province of China and Peninsular Malaysia and based on the revealed differences described the Yunnan population as a new species P. menglaensis. Wang et al. (2020) postulated that P. menglaensis is endemic to China, but suggested that this species also may occur in the surrounding low mountainous areas of neighboring Laos and Myanmar. However, our analyses have demonstrated that the distribution of this lineage is much wider and covers the entire territory of the mainland Indochina, including the type localities of Pareas berdmorei Theobald, 1868 (Mon State, southern Myanmar), and of Amblycephalus carinatus unicolor Bourret, 1934 (Kampong Speu Province, eastern Cambodia), while the Yunnan population of "P. menglaensis" is deeply nested within this radiation (Fig. 3). We thus conclude that the name Pareas berdmorei Theobald, 1868, being the eldest available synonym, has to be applied to the mainland populations of $P$. carinatus species group, while

Peer) reviewing PDF | (2021:08:64561:2:0:NEW 8 Dec 2021) 
1519

1520

1521

1522

1523

1524

1525

1526

1527

1528

1529

1530

1531

1532

1533

1534

1535

1536

1537

1538

1539

1540

1541

1542

1543

1544

1545

1546

1547

1548

1549

Amblycephalus carinatus unicolor Bourret, 1934 and Pareas menglaensis Wang, Che, Liu, Ki, Jin, Jiang, Shi \& Guo, 2020 represent subjective junior synonyms of this taxon.

Altogether, based on the concordant results of morphological and molecular analyses we report that three geographically restricted lineages exist within P. berdmorei (lineages 1-3, Fig. 3). Despite the significant morphological variation within P. berdmorei, these lineages can be readily distinguished from each other by a number of chromatic and scalation characters. We propose to recognize three subspecies within P. berdmorei: P. b. berdmorei stat. nov. from Thailand, southern Myanmar and southern China (including P. menglaensis as a junior subjective synonym), P. b. unicolor comb. nov. from southern Vietnam and Cambodia, and a new subspecies $P$. b. truongsonicus ssp. nov. for populations from the Northern Annamite Mountains in Vietnam and Laos described below.

\section{Pareas berdmorei berdmorei Theobald 1868 stat. nov.}

Figures 6C-D, 10, 13A-D; Supplementary Tables S11-S12.

Pareas berdmorei Theobald, 1868: 63.

\section{Chresonymy and synonymy:}

Pareas carinatus - (in part) Smith, 1943; Taylor, 1965; Yang \& Rao, 2008; Wallach, Williams \& Boundi, 2014; Chan-ard et al., 2015.

Pareas menglaensis Wang, Che, Liu, Ki, Jin, Jiang, Shi \& Guo, 2020.

Updated diagnosis: Pareas berdmorei berdmorei differs from other subspecies of $P$. berdmorei by the combination of the following morphological characters: body size large (TL 451-770 mm); anterior pair of chin shields wider than long; loreal and prefrontal not contacting the eye; two suboculars; one postocular; temporals generally $3+4$ (rarely $3+3$, or $2+3$ ); three median vertebral scale rows slightly enlarged; 9 infralabial scales; 15 dorsal scale rows slightly keeled in 5-13 scale rows at midbody; 166-186 ventrals; 57-89 subcaudals, all divided; dorsum light brown to yellowish with distinct dark vertebral spots and 64-72 transverse dark brownish or blackish bands (Fig. 13A-D); upper postorbital stripes weak generally not contacting each other on the nuchal area forming a narrow X-shaped dark marking with curved branches; ventral scales yellowish, generally immaculate, iris uniform from golden-bronze to orange (Fig. 13A-D).

Variation: Measurements and scalation features of the subspecies P. b. berdmorei $(\mathrm{n}=23)$ are summarized in Supplementary Table S12. There is a certain variation among sexes observed 
1550 in TaL/TL ratio and the number of subcadals scales: males have generally higher number of 1551 subcadals than females (73-89, average $78.33 \pm 6.67, \mathrm{n}=6$, in males vs. 57-82 average $155270.67 \pm 7.09, \mathrm{n}=12$ in females). In coloration, the specimens of $P$. b. berdmorei showed variation 1553 in iris color: golden-bronze iris in specimens from eastern Thailand (Fig. 13C); to orange iris in 1554 specimens from southern Yunnan and northern Thailand (Fig. 13A-B, D). Specimens also varied 1555 in the arrangement of dark markings in the nuchal area: thicker dark-brown to black markings in 1556 specimens from Thailand (Fig. 13A-C); less distinct dark markings in specimens from Laos and 1557 southern Yunnan (Fig. 13D). Other morphological features showed no significant variation 1558 among the specimens of the series examined.

1559 Distribution: Southeastern Myanmar, Northern peninsular Thailand north of the Isthmus 1560 of Kra, western, northern and eastern mainland Thailand, northern Laos, northern Vietnam, 1561 southernmost Yunnan Province of China; the southernmost known locality is in Suan Phueng District, Ratchaburi Province, Thailand (Fig. 1).

Recommended English name and Etymology: as for Pareas berdmorei.

Ecology notes: In Suan Phueng area (locality 14, Fig. 1), P. b. berdmorei occurs in sympatry with $P$. c. tenasserimicus ssp. nov., though the two taxa are restricted to different habitats (see the account for $P$. c. tenasserimicus ssp. nov. for details). Across its range, $P$. $b$. berdmorei occurs in sympatry with various congeners, including $P$. margaritophorus, $P$. macularius, P. geminatus, and P. xuelinensis. Amblycephalus carinatus unicolor Bourret, 1934: 15.

Chresonymy:

Pareas carinatus unicolor - Nguyen, Ho \& Nguyen, 2009.

Holotype: MNHN 1938.0149, adult female collected by R. Bourret from "Kompong Speu" (indicated as "Kompong Pseu" on the original label, now Kampong Spoe), Kampong Spoe Prov., eastern Cambodia.

Updated diagnosis: Pareas berdmorei unicolor comb. nov. differs from other subspecies P. berdmorei by the following combination of morphological characteristics: body size medium 1580 to small (TL 459-576 mm); anterior pair of chin shields slightly longer than broad; loreal and 
1581 prefrontal not contacting the eye; two (rarely one) suboculars; two (rarely one) postoculars; 1582 temporals generally 3+3 (rarely 3+4); three median vertebral scale rows slightly enlarged; 9 1583 infralabial scales; 15 dorsal scale rows slightly keeled in 3-9 scale rows at midbody; 162-180 1584 ventrals; 57-75 subcaudals, all divided; dorsum uniform yellow-ochre to bright orange lacking 1585 distinct dark vertebral spots and transverse dark bands (Fig. 13E-F); upper postorbital stripes 1586 generally absent or weakly discernable not contacting each other on the nuchal area; ventral scales yellowish to orange, generally immaculate, iris uniform bright orange-red (Fig. 13E-F).

Description of the holotype (MNHN 1938.0149): Adult female, specimen partially 1589 dehydrated due to preservation in ethanol for a long time (Fig. 11), body slender and notably flattened laterally; head comparatively large, narrowly elongated, clearly distinct from thin neck, snout blunt; eye rather large, pupil vertical and slightly elliptical.

Body size. SVL 390 mm; TaL 96 mm; TL 486 mm; TaL/TL: 0.198.

Body scalation. Dorsal scales in 15-15-15 rows, slightly keeled in seven scale rows at 1594 midbody, and without apical pits; three median vertebral scale rows slightly enlarged; the 1595 outermost dorsal scale row not enlarged; ventrals 164 (+ 2 preventrals), lacking lateral keels; subcaudals 64, all divided; cloacal plate single.

Head scalation. Rostral not visible from above; nasal entire; two internasals, much wider than long, narrowing and slightly curving back laterally (in dorsal view), anteriorly in contact with rostral, laterally in contact with nasal and loreal, posteriorly in contact with prefrontal, not contacting preocular; two large pentagonal prefrontals, much larger than internasals and with a slightly diagonal suture between them, not contacting the eye; single hexagonal frontal scale with its lateral sides parallel to the body axis, frontal longer than wide, smaller than parietals; two preoculars; one subocular; one postocular, not fused with subocular; one loreal in contact with prefrontal, not touching the eye; $7 / 7$ supralabials, $3^{\text {rd }}$ to $5^{\text {th }}$ SL touching the subocular, none of them reaching the eye, $7^{\text {th }}$ by SL the largest, elongate; temporals $3+3 ; 8 / 8$ infralabials, the anterior most in contact with the opposite along midline, bordering mental, anterior 5 pairs of infralabials bordering anterior chin shields; 3 pairs of chin shields interlaced, no mental groove 1608 under chin and throat; anterior chin shields relatively large, slightly longer than broad, followed by the two pairs of chin shields that are much broader than long.

Coloration. Due to preservation in ethanol for almost a century, the color pattern has 1611 significantly faded; as the consequence the specimen no longer retains the original coloration 
1612 characteristics. Presently the specimen is uniform dark reddish-brown with no pattern discernable 1613 on the ground color (Fig. 11). The original description contains the following information on the 1614 type specimen coloration: "the color is light reddish brown, absolutely uniform, without any 1615 spots on the body or head, yellower and lighter below" (Bourret, 1934: 15).

Variation: Measurements and scalation features of the subspecies Pareas berdmorei unicolor comb. nov. $(\mathrm{n}=9)$ are presented in Supplementary Table S11. There is a certain sexual dimorphism observed in body size and the number of subcadal scales: males (TL 505.0-576.0 $\mathrm{mm}$, average $552.2 \pm 40.9 \mathrm{~mm}, \mathrm{n}=3$ ) have slightly larger body size than females (TL 459.0-538.1 $\mathrm{mm}$, average $498.4 \pm 32.6 \mathrm{~mm}, \mathrm{n}=6$ ); males also have a generally higher number of subcadals than females (73-75, average $73.7 \pm 1.2, \mathrm{n}=3$ in males vs. $58-75$, average $66.3 \pm 5, \mathrm{n}=6$ in females). Coloration of the examined specimens varied in the ground color (from yellow-ochre to bright orange), and in the dark markings on the head and nuchal area (from upper postorbital stripes absent to weakly discernable). Other morphological features showed no significant variation among the examined specimens.

Comparisons: Pareas berdmorei unicolor comb. nov. differs from $P$. b. berdmorei by slightly lower number of ventrals (162-180 [average 173.6 \pm 5.1 ] vs. 166-186 [average 178.10 \pm 5.19 ]), by generally lower number of keeled dorsal scale rows (3-9 [average 6.8 \pm 1.9 ] vs. 5-13 [average 9.6 \pm 2.2 ]), and by uniform orange to beige coloration lacking dark markings and transverse bands (vs. present) and brighter orange-red coloration of iris (vs. golden-bronze to orange). Pareas berdmorei unicolor comb. nov. differs from $P$. b. truongsonicus ssp. nov. described below by slightly smaller total length (459-576 mm [average $516.3 \pm 42.5 \mathrm{~mm}$ ] vs.

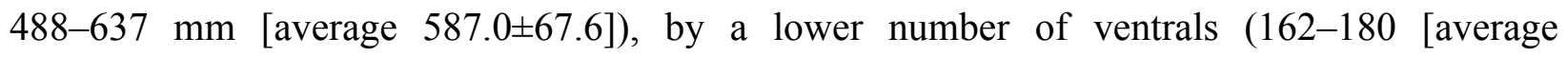
$173.6 \pm 5.1$ ] vs. 167-187 [average 179.5 \pm 9.6 ]), by lower number of keeled dorsal scale rows (3-9 [average 6.8 \pm 1.9 ] vs. 13), and by uniform orange to beige coloration lacking dark markings and transverse bands (vs. dark markings present) and brighter orange-red coloration of iris (vs. offwhite to golden). For the detailed comparisons of the three subspecies of Pareas berdmorei see Supplementary Table S12.

Distribution: Based on our morphological and molecular data, P. b. unicolor comb. nov. inhabits the lowland and foothill tropical forests of southern Vietnam and eastern Cambodia (Fig. 1). The actual extend of the subspecies distribution in central Vietnam and central Cambodia is 1642 still unclear and requires further survey efforts. 
1643

1644

1645

1646

1647

1648

1649

1650

1651

1652

1653

1654

1655

1656

1657

1658

1659

1660

1661

1662

1663

1664

1665

1666

1667

1668

1669

1670

1671

1672

1673

Etymology: The subspecies name "unicolor" is a Latin adjective in nominative singular meaning "monochrome" and was given in reference to the uniform coloration of this snake.

Recommended English name: Cochinchinese slug-eating snake.

Ecology notes: In Di Linh District, Lam Dong Province of southern Vietnam (locality 37, Fig. 1), P. b. unicolor comb. nov. occurs in sympatry with $P$. temporalis described below; these two taxa were recorded in the same habitat within the mid-elevation evergreen tropical forests of the Langbian Plateau (see the account for P. temporalis for details). Across its range, $P$. $b$. unicolor occurs in sympatry with other congeners, including P. margaritophorus, $P$. macularius, and $P$. formosensis.

\section{Pareas berdmorei truongsonicus Poyarkov, Nguyen TV, Vogel, Brakels \& Pawangkhanant ssp. nov. \\ [urn:1sid:zoobank.org:act:3E45EE5B-8DD5-4FB1-814A-76DC2B821E29] \\ Figures 6E, 12, 13G-H; Supplementary Tables S11-S12.}

\section{Chresonymy:}

Pareas carinatus - (in part) Ziegler et al., 2006, 2016; Nguyen, Ho \& Nguyen, 2009; Teynié \& David, 2010; Le et al., 2014; Pham \& Nguyen, 2019.

Holotype: ZMMU R-16801 (field number NAP-09150), adult female collected by N. A. Poyarkov, P. Brakels, P. Pawangkhanant and T. V. Nguyen from limestone forest near the Tham Mangkon Cave, in Ban Nahin-Nai District, Khammouan Province, central Laos (N 18.22111, E 104.81243; elevation 526 m asl.) on July 14, 2019.

Paratype: ZMMU R-14796, adult male collected by N. A. Poyarkov and N. L. Orlov from limestone forest in environs of Kim Lich Village, Kim Hoa Commune, Tuyen Hoa District, Quang Binh Province, central Vietnam (N 18.01206, E 105.92215; elevation $41 \mathrm{~m}$ asl.) on September 7, 2015.

Referred specimens: ZFMK 82890, adult male collected on July 5, 2014, and VNUH 15.6.'05-1 collected on June 15, 2005 from Phong Nha - Ke Bang National Park, Quang Binh Province, Vietnam (see Ziegler et al., 2006).

Diagnosis: Pareas berdmorei truongsonicus ssp. nov. differs from other subspecies of $P$. berdmorei by the combination of the following morphological characters: maximal total length of $637 \mathrm{~mm}$; anterior pair of chin shields as long as broad; loreal and prefrontal not contacting the 
1674 eye; one subocular; one postocular; temporals 3+4; three median vertebral scale rows slightly 1675 enlarged; 9 infralabial scales; 15 dorsal scale rows keeled in 13 scale rows at midbody; 167-187

1676

1677

1678

1679

1680

1681

1682

1683

1684

1685

1686

1687

1688

1689

1690

1691

1692

1693

1694

1695

1696

1697

1698

1699

1700

1701

1702

1703

1704

ventrals; 66-80 subcaudals, all divided; dorsum light brown with distinct dark-brown vertebral spots and 68-71 transverse dark bands, and with dense brownish-gray mottling covering dorsal, lateral and ventral surfaces of body and head (Fig. 13G-H); upper postorbital stripes discernable, contacting each other on the nuchal area forming a clear Y-shaped pattern; ventral scales yellowish-white with dense brownish mottling, iris uniform off-white to golden (Fig. 13G-H).

Description of the holotype (ZMMU R-16801): Adult female, specimen in a good state of preservation (Fig. 12), body slender and notably flattened laterally; head large, narrowly elongated and flattened, clearly distinct from thin neck, snout blunt; eye large, pupil vertical and slightly elliptical.

Body size. SVL 499 mm; TaL 123 mm; TL 622 mm; TaL/TL: 0.198.

Body scalation. Dorsal scales in 15-15-15 rows, the medial 13 scale rows slightly keeled at midbody, all dorsal scales lacking apical pits; three median vertebral scale rows enlarged; outermost dorsal scale row not enlarged; ventrals 187 (+ 1 preventral), all lacking lateral keels; subcaudals 66; cloacal plate single.

Head scalation. Rostral not visible from above; nasal single; internasals two, much wider than long, narrowing and slightly curving back laterally (in dorsal view), anteriorly in contact with rostral, laterally in contact with nasal and loreal, posteriorly in contact with prefrontal, not contacting preocular; two large irregular pentagonal prefrontals, much larger than internasals and with a slightly diagonal suture between them, not contacting the eye; the single frontal scale hexagonal with the lateral sides parallel to the body axis, longer than wide, smaller than parietals; one preocular; one subocular; one postocular, not fused with subocular; one loreal in contact with prefrontal, not touching the eye; $7 / 7$ supralabials, $3^{\text {rd }}$ to $5^{\text {th }}$ SL touching the subocular, none of them reaching the eye, $7^{\text {th }}$ SL the largest, elongate; temporals $3+4 ; 9 / 9$ infralabials, the anterior most in contact with the opposite along the midline, bordering mental, anterior 5 pairs of infralabials bordering the anterior chin shields; 3 pairs of chin shields interlaced, no mental groove under chin and throat; anterior chin shields relatively large, as long as broad, followed by two pairs of chin shields that are much broader than long.

Coloration. In life, dorsal surfaces of the head brownish with numerous marbled markings and dense brownish mottling (Fig. 13H). Head with two lateral postorbital stripes: the lower one

Peer] reviewing PDF | (2021:08:64561:2:0:NEW 8 Dec 2021) 
1705 is thick dark-brown line continuing from the middle of the eye onto the anterior part of the last 1706 supralabial; the upper one is a slightly thinner dark line running from postocular backwards to the 1707 dorsal scales on the neck (Fig. 12D). The upper postorbital stripes from the both sides of the 1708 body meet each other in the nape area forming a dark Y-shaped pattern (Fig. 13H). Lateral and 1709 ventral surfaces of the head marked with a dense brown dusting and larger dark spots (Fig. 12ED). Dorsal surfaces light-brown with ca. 68 faint vertical dark brown bands. Ventral surfaces of the head, body and tail yellowish-cream with dense brown dusting. Iris uniform off-white, pupil black. In preservative: After two years of storage in ethanol the general coloration pattern has not changed (Fig. 12); yellowish tint in the coloration of dorsum, the head and eyes have faded becoming grayish-brown; other coloration features remain unchanged.

Variation: Measurements and scalation features of the subspecies Pareas berdmorei truongsonicus ssp. nov. $(\mathrm{n}=4)$ are presented in Supplementary Table S11. The two specimens examined in our study have a very similar coloration with the two specimens from Phong NhaKe Bang N. P. (ZFMK 82890 and VNUH 15.6.'05-1) reported by Ziegler et al. (2006); this population is geographically close (ca. $50 \mathrm{~km}$ direct distance; see Fig. 1, c) to the loaclity of the new subspecies in Kim Hoa Commune, Tuyen Hoa District, Quang Binh Province. However, the members of the type series of the new subspecies have slightly lower number of ventral and subcaudal scales as compared to the specimens reported in Ziegler et al. (2006): VEN 187 vs 167-177; SC 66-73 vs. 78-80. We also report on a population from Xe Pian N.P.A., Champasak Province, Laos (Fig. 1, b) which agrees well with the specimens examined in our study in coloration (Fig. 13, G); the morphological or genetic data on this population is lacking. Therefore, in this study we tentatively assign the specimens from Phong Nha-Ke Bang N.P. and from Xe Pian N.P.A. to $P$. cf. $b$. truongsonicus ssp. nov.; the taxonomic status of this population has to be clarified in the future.

Comparisons: In our sample of four specimens Pareas berdmorei truongsonicus ssp. nov. differs from P. b. berdmorei by slightly higher of number of ventrals (187 vs. 166-186

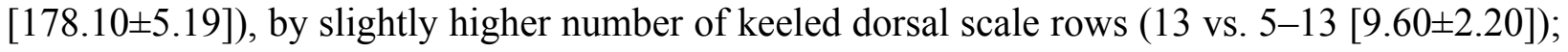
by the dense brownish mottling and bigger brown spots on dorsal, lateral, and ventral surfaces of the head and body (vs. ventral surfaces immaculate, lateral and dorsal surfaces with sparse dusting); and by uniform off-white to golden color of iris (vs. golden-bronze to orange). The new 
$1736637 \mathrm{~mm}$ vs. $459-576 \mathrm{~mm}$ [516.3 $\pm 42.5 \mathrm{~mm}])$, by a generally higher number of ventrals (187 vs.

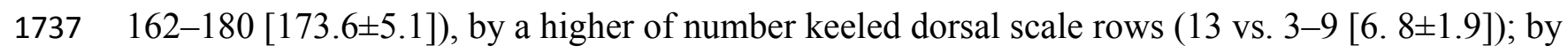

1738 the presence of dark markings on dorsum and ventral surfaces, including the dark transverse

1739 bands and brownish mottling (vs. uniform orange to beige coloration lacking dark markings),

1740 and by off-white to golden coloration of iris (vs. bright orange-red color of iris). Detailed

1741 comparisons of the three subspecies of Pareas berdmorei are presented in Supplementary Table

$1742 \mathrm{~S} 12$.

1743 Distribution: To date the new subspecies is known only from the northern portion of the 1744 Annamite (Truong Son) Mountain Range in central Vietnam and eastern Laos (localities 27-28, 1745 Fig. 1).

1746 Etymology: The new subspecies name "truongsonicus" is a Latin toponymic adjective in 1747 nominative singular, adopting the masculine gender of the genus name Pareas, and is given in 1748 reference to the Truong Son (Annamite) Mountain Range in Vietnam and Laos, where the new 1749 subspecies occurs.

1750 Recommended English name: Annanmite slug-eating snake.

1751 Ecology notes: In Tuyen Hoa District, Quang Binh Province of Vietnam, where the 1752 paratype of the new subspecies was collected, it was recorded in sympatry with $P$. 1753 margaritophorus and $P$. formosensis. This taxon seems to be associated with karst evergreen 1754 forests (Ziegler et al., 2006; this study). As other members of the genus Pareas, Pareas 1755 berdmorei truongsonicus ssp. nov. is a nocturnal semi-arboreal snake, all specimens were 1756 recorded while crawling on branches of bushes ca. $1 \mathrm{~m}$ above the ground or on limestone rocks; 1757 the diet is unknown but it likely includes terrestrial mollusks.

1758

1759

1760

1761

1762

1763

1764

1765

1766

Pareas kuznetsovorum Poyarkov, Yushchenko \& Nguyen TV sp. nov. [urn:Isid:zoobank.org:act:1CD26CB3-F3E9-4370-B501-6F678851C9FB]

Figures 6G, 14-15; Table 2; Supplementary Tables S11, S13.

Holotype: ZMMU R-16802 (field number NAP-10333), adult male collected by N. A. Poyarkov from the lowland semideciduous monsoon forest in Song Hinh Protected Forest, Song Hinh District, Phu Yen Province, southern Vietnam (N 12.77522, E 109.04606; elevation 583 m asl.) on January 16, 2021.

Diagnosis: Pareas kuznetsovorum sp. nov. differs from other members of the genus 
1767 Pareas by the combination of the following morphological characteristics: total length up to 639 $1768 \mathrm{~mm}$; anterior pair of chin shields longer than broad; loreal and prefrontal not contacting the eye; 1769 two suboculars; one postocular; temporals 3+4; the single median vertebral scale row slightly 1770 enlarged; 7 supralabial scales; 7-8 infralabial scales; 15 dorsal scale rows, all smooth; 167 1771 ventrals; 87 subcaudals, all divided; dorsum tan to light brown with distinct dark-brown vertebral 1772 line, blackish vertebral spots and 70 transverse dark-brown bands; upper postorbital stripes thick, 1773 black, contacting each other on the nuchal area forming a dark black $\Psi$-shaped chevron; lower 1774 postorbital stripes thin, black, reaching the anterior part of SL7, not continuing to the lower jaw 1775 and chin; belly yellow with sparce dark-gray dusting and brown elongated spots forming three longitudinal lines on ventrals; iris uniform off-white with beige lateral parts.

Description of the holotype (ZMMU R-16802): Adult male, specimen in a good state of preservation (Fig. 14); body slender and notably flattened laterally; head large, narrowly elongated, clearly distinct from thin neck (head more than two times wider than neck width near the head basis); snout blunt; eye rather large, pupil vertical and elliptical.

Body scalation. Dorsal scales in 15-15-15 rows, all scales smooth and lacking apical pits; vertebral scales slightly enlarged; the outermost dorsal scale row not enlarged; ventrals 167 (+ 1 preventral), lacking lateral keels; subcaudals 87, all divided; cloacal plate single.

Head scalation. Rostral not visible from above; nasal single; two internasals, much wider than long, narrowing and slightly curving back laterally (in dorsal view), anteriorly in contact with rostral, laterally in contact with nasal and loreal, posteriorly in contact with prefrontal, not contacting preocular; two large pentagonal prefrontals, much larger than internasals and with a straight suture between them, not in contact with eye; one hexagonal frontal, longer than wide, with the lateral sides parallel to the body axis, roughly the same size as the parietals; single preocular; single postocular, semicrescentic in shape, not fused with subocular; two suboculars; single loreal, in contact with preocular, prefrontal, internasal and nasal, not touching the eye; 7/7 supralabials, $3^{\text {rd }}$ to $5^{\text {th }}$ SL touching the subocular, none of them reaching the eye, $7^{\text {th }}$ by far the largest, elongated; $1 / 1$ supraoculars; $3 / 3$ anterior temporals and 3/4 posterior temporals; 8/7 infralabials, the anteriormost in contact with opposite along midline forming a straight suture, bordering mental, the anterior 5 pairs of infralabials bordering the anterior chin shields; 3 pairs of chin shields interlaced, no mental groove under chin and throat; anterior chin shields relatively 
1798 large, notably longer than broad, followed by the two pairs of chin shields that are much broader 1799 than long.

Hemipenial morphology. Fully everted hemipenis symmetrical, bilobed, forked (Fig. 15B-

1801

1802

1803

1804

1805

1806

1807

1808

1809

1810

1811

1812

1813

1814

1815

1816

1817

1818

1819

1820

1821

1822

1823

1824

1825

1826

1827

1828

C); the surface from base to crotch smooth, with several (5-6) weakly discernable dermal ridges on the asulcal surface (Fig. 15C) and few (3-4) shallow folds on the sulcal surface (Fig. 15B). Sulcus spermaticus deep, with fleshy swollen edges, bifurcate into two separate canals towards or on the apical lobes. Apical lobes curved with well-developed ornamentation, covered with large fleshy transverse occasionally interwining folds, separated with deep slits and forming a complex pattern resembling the bellows of an accordion (Fig. 15B-C).

Coloration. In life, the dorsal surfaces of the head brownish with dense darker marbling (Fig. 15A). Head laterally off-white with dark-brown spots and blotches, ventrally yellowishwhite with few small black spots. Head with two lateral postorbital stripes: the lower one is a thin black line starting from the posterior portion of subocular and running ventrally and posteriorly towards lower temporals to the posterior part of the $6^{\text {th }}$ supralabial and further to the anterior part of $7^{\text {th }}$ supralabial; the upper one is a well-defined thick black line starting from the upper part of postocular backwards to the dorsal scales of neck (Fig. 14C-D), where it joins a large rectangular black spot covering the nape, overall forming a dark black $\Psi$-shaped chevron pattern (Fig. 14E). Upper labials marked with a dense brown dusting. Dorsal surfaces of the body tan to light brown with a distinct dark-brown line running along the vertebral scale row, and with about 70 black vertebral spots and transverse dark-brown bands (Fig. 14A-B); ventral surfaces of the head, body and tail yellowish with sparce dark-gray dusting and brown elongated spots forming three longitudinal lines on ventrals (Fig. 14A-B). Iris uniform off-white with beige lateral parts; pupil black (Fig. 14C-D). In preservative: After six months of storage in ethanol the general coloration pattern has not changed; the tan coloration of dorsum slightly faded becoming light grayish-brown, light coloration on head and iris faded becoming brownish; other features of coloration remain unchanged.

Comparisons: Pareas kuznetsovorum sp. nov. differs from P. berdmorei by all dorsal scales smooth (vs. 3-13 dorsal scale rows keeled), higher number of subcaudals (87 vs. 63-78 [average 71.13 \pm 7.23$]$ ), by the presence of black chevron on the nuchal area (vs. absent); the new species further differs from $P$. carinatus by the presence of two postoculars (vs. single or absent); by a generally higher number of subcaudals ( 87 vs. 54-96 [average $69.24 \pm 8.98$ ]), by all 
1829 dorsal scale rows smooth (vs. 3-11 dorsal scale rows keeled [average 6.52 \pm 2.94 ]), by the 1830 presence of black nuchal chevron (vs. absent); and by a lower number of enlarged vertebral scale 1831 rows ( 1 vs. 3 [average 2.83 \pm 0.56 ]); it further differs from P. nuchalis (Boulenger) by prefrontal

1832

1833

1834

1835

1836

1837

1838

1839

1840

1841

1842

1843

1844

1845

1846

1847

1848

1849

1850

1851

1852

1853

1854

1855

1856

1857

1858

1859

not contacting the eye (vs. in contact); by lower number of ventrals (167 vs. 201-220 [average $209.89 \pm 5.25]$ ); and by a lower number of subcaudals (87 vs. 102-120 [average 111.11 \pm 6.05 ]).

Morphological comparisons between all species of the subgenus Pareas are detailed in Supplementary Table S13.

Distribution: To date Pareas kuznetsovorum sp. nov. is known only from the type locality in the north-eastern foothills of the Langbian Plateau in Phu Yen Province of Vietnam (locality 41, Fig. 1). Though only single specimen of the new species is known up to date, its occurrence is expected in the remaining fragments of lowland to mid-elevation evergereen forests of the north-western slopes of the plateau, particularly in the adjacent parts of Dak Lak and Khanh Hoa provinces of southern Vietnam.

Etymology: The new species name "kuznetsovorum" is the plural possessive form of the family name Kuznetsov. This species is named in honor of two biologists, Andrei N. Kuznetsov and Svetlana P. Kuznetsova. They have greatly contributed to organization of biological expedtions of the Joint Russian-Vietnamese Tropical Center in various parts of Vietnam from 1996 to 2021; without their enthusiasm and support our fieldwork in Vietnam, including the expedition during which the holotype of the new species was collected, would have not been possible.

Recommended English name: Kuznetsovs' slug-eating snake.

Ecology notes: The single specimen of the new species was collected in middle January during the period where most of reptile species were not active; the specimen was recorded at 00:00 h while perching on a Calamus sp. palm leaf near a forest trail ca. $1.5 \mathrm{~m}$ above the ground, when the air temperature was around $12^{\circ} \mathrm{C}$ under a drizzling rain. The specimen was not moving. No other members of Pareidae were recorded in the area of survey. The diet of the new species is unknown but, as in other congeners, it presumably consists of terrestrial mollusks.

\section{Pareas nuchalis species group}

The monophyly of the nuchalis group is strongly supported in BI-analysis, and got only poor support in ML-analysis (0.99/80); this group includes P. nuchalis from Borneo and Sumatra 
1860 (lineage 9, Fig. 3) and two lineages from the montane areas in central and southern Vietnam, one 1861 of which we describe below as a new species, forming a strongly-supported group (1.0/100) 1862 (lineages 7-8, Fig. 3). The lineage inhabiting the Kon Tum - Gia Lai Plateau in central Vietnam 1863 represents Pareas abros sp. nov. (lineage 7, Fig. 3), and the second lineage from Langbiang 1864 Plateau in southern Vietnam corresponds to the recently described P. temporalis (lineage 8, Fig. 1865 3). All members of the nuchalis group are characterized by the presence of a large black ringshaped blotch on the nape, connected to the upper and lower postorbital stripes anteriorly.

\section{Pareas nuchalis (Boulenger, 1900)}

Amblycephalus nuchalis Boulenger, 1900: 185.

Chresonymy:

Amblycephalus nuchalis —De Rooij, 1917: 277.

Pareas nuchalis — Malkmus \& Sauer, 1996; Malkmus et al., 2002; Wallach, Williams \& Boundi, 2014: 537.

Pareas carinatus — (in part) David \& Vogel, 1996.

Holotype: NHMUK 1912247 (formely BMNH 1901.5.14.2), adult male from Saribas, Betong Division, State of Sarawak, Borneo, Malaysia (approximately N 1.410, E 111.527; elevation $15 \mathrm{~m}$ asl.), collected by A. H. Everett.

Updated diagnosis: Pareas nuchalis differs from other members of the genus Pareas by the following combination of morphological characters: maximal total length up to $678 \mathrm{~mm}$; anterior pair of chin shields longer than broad; loreal not contacting the eye; prefrontal in contact with the eye; 1-3 suboculars; 1-2 postoculars; temporals generally $3+4$ or $3+3$; one to three median vertebral scale rows slightly enlarged; 7-8 supralabial scales; generally 7 (rarely 6 or 8) infralabials; 15 dorsal scale rows at midbody, all totally smooth; 201-220 ventrals; 102-120 subcaudals, all divided; dorsum tan to light brown with weak dark-brown vertebral spots and 6178 distinct transverse dark-brown bands (Figs. 16-17); upper postorbital stripes thick, black, bifurcating posterior to the secondary temporals, forming a vertical black bar to the mouth angle; upper postorbital stripes contacting each other on the nuchal area forming a large black ringshaped blotch (Fig. 17); lower postorbital stripes thick, black, reaching the anterior part of SL6, 
1891 dusting (Figs. 16-17); iris in life whitish with brownish speckles and veins getting denser around 1892 the pupil (Fig. 17) (Boulenger, 1900; Stuebing et al., 2014; our data).

Material examined: In this study we directly examined nine specimens of Pareas nuchalis from Borneo (Malaysia, Indonesia) and Sumatra, including the holotype of Amblycephalus nuchalis (see Supplementary Table S11, Appendix II).

Coloration. Due to preservation in ethanol for more than a century, the coloration and the pattern of the holotype has been changed, as the consequences the type specimen no longer retains the original coloration characteristics (Fig. 16). Dorsal surface of the head uniform dark brown, head with two postorbital stripes, the upper running laterally backwards to the dorsal scales on the neck, bifurcating posterior to the secondary temporal scales, forming a dark vertical bar reaching the mouth angle, upper postorbital stripes contacting each other on the nuchal area forming a large black ring-shaped blotch (Fig. 16); lower postorbital stripes partially faded, reaching the anterior part of SL6. Dorsal surface of the body light brown with 78 vertical faint dark-brown bands; ventral surface of the body and tail yellowish-cream with sparse brown 1905 dusting.

Variation: Measurements and scalation features of Pareas nuchalis specimens examined $(n=9)$ are presented in Supplementary Table S11. There is a certain sexual variation observed in the body size, numbers of ventral and subcadal scales: males have slightly lager total length (TL 345.0-678.0 mm, average 529.0 $\pm 110.2 \mathrm{~mm}, \mathrm{n}=6$ ) than females (TL 352.0-503.0 mm, average $422.3 \pm 76.0 \mathrm{~mm}, \mathrm{n}=3$ ); males also have generally slightly higher number of ventral and subcaudal scales than females (VEN 207-220, average 212.2 \pm 4.5 , $\mathrm{n}=6$; SC 108-120, average 114.2 \pm 4.7 , $\mathrm{n}=6$ in males vs. VEN 201-208, average 205.3 $\pm 3.8, \mathrm{n}=3$; SC 102-107, average 105.0 $\pm 2.7, \mathrm{n}=3$ in females). Other morphological and coloration features showed no significant variation among the specimens of the examined series.

Distribution: Until recently, this species was considered to be endemic to the island of Borneo (Stuebing et al., 2014), and was recorded both from the State of Sarawak and Sabah in Malaysia, Brunei and from the Indonesian part of the island (Kalimantan). In this study we for the first time recorded $P$. nuchalis from central Sumatra, where it was previously confused with 1919 P. carinatus (see David \& Vogel, 1996). 
Etymology: The species name "nuchalis" is a Latin adjective in nominative singular

1921

1922

1923

1924

1925

1926

1927

1928

1929

1930

1931

1932

1933

1934

1935

1936

1937

1938

1939

1940

1941

1942

1943

1944

1945

1946

1947

1948

1949

1950

meaning "nuchal" and was given in reference to the characteristic black ring-shaped spot in the nuchal area in this species.

Recommended English name: Barred slug-eating snake.

\section{Pareas abros Poyarkov, Nguyen TV, Vogel \& Orlov sp. nov.} [urn:1sid:zoobank.org:act:85CA3212-E8D4-48D1-8ED2-DC8CB183E7E9]

Figures 6I, 18-19; Table 2; Supplementary Tables S11, S13.

Holotype: ZMMU R-16393 (field number NAP-08867), adult male collected by N. A. Poyarkov from the montane evergreen tropical forest near the offsprings of the Paete River, within the Song Thanh N.P., La Dee Commune, Nam Giang District, Quang Nam Province, central Vietnam (N 15.53353, E 107.38434; elevation 1083 m asl.) on May 05, 2019.

Paratypes: ZMMU R-16392 (field number NAP-06251), adult male, and ZMMU R-14788 (field number NAP-06252), adult female, both collected by N. A. Poyarkov and N. L. Orlov from the montane evergreen tropical forest within the Sao La Nature Reserve, A Roang area, A Luoi Distict, Thua Thien - Hue Province, central Vietnam (N 16.10334, E 107.444453; elevation 796 m asl.) on September 11-17, 2015.

Diagnosis: Pareas abros sp. nov. differs from all other members of the genus Pareas by the combination of the following morphological characters: body size medium (TL 434-565 $\mathrm{mm}$ ); head notably flattened dorsoventrally; anterior pair of chin shields longer than broad; loreal and prefrontal not contacting the eye; three suboculars; two postoculars; temporals $3+3$; the single median vertebral scale row slightly enlarged; 9 supralabial scales; generally 8 (rarely 9) infralabials scales; 15 dorsal scale rows at midbody, of them 9-11 median scale rows slightly keeled; 180-184 ventrals; 83-95 subcaudals, all divided; dorsum yellowish-brown with distinct dark-brown vertebral line, barely distinct blackish vertebral spots and 44-56 faint interrupted transverse dark-brown bands (Fig. 19A-C); upper postorbital stripes thick, slate-black, bifurcating posterior to the secondary temporals, forming a thick black line, continuing to the $7^{\text {th }}$ SL and further on the neck; upper postorbital stripes contacting each other on the nuchal area forming a large ring-shaped blotch (Fig. 19A-C); two thick, black lower postorbital stripes reaching the $6^{\text {th }}$ and $8^{\text {th }} \mathrm{SL}$, and continuing to the lower jaw; belly beige with dense brownishgray dusting and dark brown elongated spots forming two longitudinal lines on the lateral sides

Peer] reviewing PDF | (2021:08:64561:2:0:NEW 8 Dec 2021) 
1951 of ventrals (Fig. 18D); iris in life beige with ochraceous to orange speckles and veins getting 1952 denser around the pupil (Fig. 19).

1953 Description of the holotype (ZMMU R-16393): Adult male, specimen in a good state of 1954 preservation (Fig. 18); body slender and notably flattened laterally; head very large, notably 1955 flattened dorsoventrally, clearly distinct from thin neck (head more than two times wider than 1956 neck near the head basis), snout obtusely rounded in profile and in dorsal view; eye very large, 1957 pupil vertical and elliptical. Hemipenis not everted.

Body size. SVL 314 mm; TaL 120 mm; TL 434 mm; TaL/TL: 0.276.

Body scalation. Dorsal scales in 15-15-15 rows, slightly keeled on 11 upper scale rows at midbody (Fig. 18F), all lacking apical pits; the single median vertebral scale row slightly enlarged; the outermost dorsal scale row not enlarged; ventrals 184 (+ 1 preventral), lacking lateral keels; subcaudals 92; cloacal plate single.

Head scalation. Rostral not visible from above; single nasal; two internasals, much wider than long, narrowing and slightly curving back laterally (in dorsal view), anteriorly in contact with rostral, laterally in contact with nasal and loreal, posteriorly in contact with prefrontal, not contacting preocular; two large irregularly pentagonal prefrontals, much larger than internasals and with a straight suture between them, not in contact with eye; the single frontal scale hexagonal with the lateral sides slightly concave, parallel to the body axis, longer than wide, smaller than parietals; single preocular; two postoculars, semicrescentic in shape, not fused with subocular; single presubocular; three suboculars; two loreals, upper larger then lower, irregularly pentagonal, in contact with presubocular, prefrontal, internasal and nasal, not touching the eye; 9/9 supralabials, $4^{\text {th }}$ to $6^{\text {th }}$ SL touching the subocular, none of them reaching the eye, $9^{\text {th }}$ by far the largest, elongated; $1 / 1$ supraoculars; $3 / 3$ anterior temporals and 3/3 posterior temporals; 8/8 infralabials, the anteriormost in contact with the opposite along midline forming a diagonal suture between them, bordering mental, the anterior five pairs of infralabials bordering the anterior chin shields; 3 pairs of chin shields interlaced, no mental groove under chin and throat; the anterior chin shields relatively large, much longer than broad, followed by two pairs of chin shields that are much broader than long.

Coloration. In life, dorsal and ventral surfaces of the head brownish with dense dark-brown mottling. Head with three lateral postorbital stripes: the upper postorbital stripes thick, slate- 
1982 posterior to the secondary temporals, forming a ventral branch - a thick black line, continuing to 1983 the $9^{\text {th }} \mathrm{SL}$ and further to the posterior corner of the jaw and on the neck; the dorsal branch 1984 extending to the top of the nape contacting each other on the nuchal area; both of the branches of 1985 the upper postorbital stripe join at the front of the neck to form a large black ring-shaped blotch that covers the entire nape area (Fig. 18C). Two lower postorbital stripes: the posterior one is a thick, black line starting from the lower portion of postorbital, running ventrally and posteriorly towards lower temporals to $8^{\text {th }}$ and $9^{\text {th }}$ supralabials, and further and continuing to the lower jaw; the anterior lower postorbital stripe is a short thick black vertical stripe starting from the middle 1990 subocular, reaching the $6^{\text {th }}$ SL and continuing further to the $4^{\text {th }}$ IL as a line of black spots (Fig. 18C). Upper labials beige with dense brown dusting. Dorsal surfaces of body yellowish-brown with distinct dark-brown line running along the vertebral dorsal scale row, barely distinct 1993 blackish vertebral spots and ca. 56 faint interrupted transverse dark-brown bands (Fig. 18A-B). Ventral surfaces of the head, body and tail cream-beige with dense brownish-gray dusting and dark brown elongated spots forming two longitudinal lines on the lateral sides of ventrals (Fig. 18D). Iris in life beige with orange speckles and veins getting denser around the pupil; pupil black (Fig. 18C). In preservative: After two years of storage in ethanol the general coloration pattern did not change; the tan tint of the dorsal coloration, and orange tints on the head and eye have faded becoming grayish-brown; other features of coloration remain unchanged.

Variation: Measurements and scalation features of the type series $(n=3)$ is presented in Supplementary Table S11. The holotype has two loreals while there is only one in the two paratypes. There is a certain variation observed in the number of ventral and dorsal scales: males have higher number of subcaudals $(92-95, \mathrm{n}=2)$ than the single female $(83, \mathrm{n}=1)$; dorsal scales are keeled in 11 scale rows at midbody in males vs. in 9 scale rows are keeled in the single female. Coloration features among the members of the type series were very similar.

Hemipenial morphology. The hemipenis is partially everted in the adult male paratype ZMMU R-16392 (Fig. 19D). The partially everted organ symmetrical, bilobed, forked, the surface from base to crotch smooth, with numerous (7-11) shallow folds and on the sulcal 2009 surface and fewer 3-6 larger dermal ridges on the asulcal surface. Sulcus spermaticus deep, with fleshy swollen edges, bifurcating into two separate canals towards the apical lobes. Apical lobes with well-developed ornamentation, covered with large fleshy irregularly curved folds in $4-5$ 
2012 rows and fleshy protuberances, separated with deep slits, forming a complex pattern resembling 2013 brain cortex.

2014 Comparisons: Pareas abros sp. nov. differs from P. berdmorei by the anterior pair of chin 2015 shields longer than broad (vs. broader than long), by slightly longer tail (TaL/TL 0.26-0.29 2016 [average $0.28 \pm 0.01$ ] vs. $0.17-0.27$ [average $0.21 \pm 0.02$ ]), by slight higher number of ventrals (83201795 [average $90.00 \pm 6.24$ ] vs. 57-89 [average 71.13 \pm 7.25 ]), by the presence of a large ring-shaped 2018 black blotch in the nuchal area (vs. absent); and by the presence of the dark vertebral line (vs. 2019 absent). The new species differs from P. carinatus by longer tail (TaL/TL 0.26-0.29 [average 2020 $0.28 \pm 0.01$ ] vs. $0.18-0.25$ [average $0.22 \pm 0.02$ ]), by a slightly higher number of subcaudals (83-95

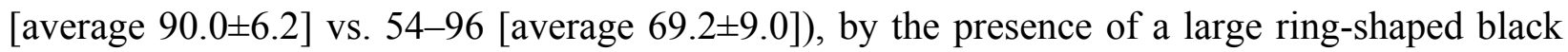
blotch in the nuchal area (vs. absent); by the presence of the dark vertebral line (vs. absent); and 2023 by weakly-discernable faint transverse dark bands on body (vs. well-discernable dark bands). Pareas abros sp. nov. differs from $P$. nuchalis by prefrontal not in contact with the eye (vs. in contact); by 9-11 dorsal scale rows keeled (vs. all dorsal scales totally smooth); and by the black nuchal blotch forming a complete ring (vs. incomplete ring-shaped blotch). The new species differs from P. kuznetsovorum sp. nov. (described above) by a higher number of ventrals (180184 [average $182.7 \pm 2.3$ ] vs. 167); by keeled 9-11 dorsal scale rows (vs. all dorsal scales totally smooth); by smaller body size (TL 434-565 mm [average 506.7 $\pm 66.7 \mathrm{~mm}$ ] vs. $639 \mathrm{~mm}$ ). Pareas 2030 abros sp. nov. differs from Pareas temporalis by a slightly lower number of ventrals (180-184 vs. 185-198), by weak keels present only on 9-11 dorsal scale rows (vs. all dorsal scale rows strongly keeled), by a lower number of enlarged vertebral scales rows (1 vs. 3), and by having 2033 44-56 faint dark transverse bands (vs. absence of dark cross-bands on the body). Morphological comparisons between all species of the subgenus Pareas are detailed in Table 2 and Supplementary Table S13.

Distribution: The new species is to date known only from two localities in Quang Nam (locality 42, Fig. 1) and Thua Thien - Hue (locality 43, Fig. 1) provinces of central Vietnam, both of them are located within the Kon Tum - Gia Lai Plateau, the northern portion of the 2039 Central Highlands (Tay Nguyen) Region of Vietnam. The Kon Tum - Gia Lai Plateau is isolated from the adjacent mountain massifs by lowland areas and is characterized by a high level of 2041 herpetofaunal endemism (Bain \& Hurley, 2011; Poyarkov et al., 2021); the new species is also 2042 likely an endemic of this mountain region. The holotype of the new species was collected in just 
$20432 \mathrm{~km}$ from the national border of Vietnam and Lao PDR (locality 42, Fig. 1), hence the 2044 occurrence of Pareas abros sp. nov. in Laos is highly anticipated.

2045 Etymology: The new species name "abros" is a Latinized adjective in nominative singular 2046 derived from the classical Greek word "abros" ( $\alpha \beta \rho o ́ \varsigma)$, meaning "cute", "handsome", and 2047 "delicate". The name is given in reference to the appealing and cute appearance of the new 2048 species, as well as other members of the genus Pareas.

Recommended English name: Cute slug-eating snake.

Ecology notes: Pareas abros sp. nov. inhabits montane evergreen tropical forests of Kon Tum - Gia Lai Plateau and was recorded on the elevations from 796 to 1,083 m asl. In both localities, the new species was recorded in fragments of primary polydominant forest along the banks of montane streams. The new species was active at 21:00 - 00:00 h, the specimens were usually located while crawling on the branches of bushes and trees ca. 1-1.5 $\mathrm{m}$ above the ground; the holotype was spotted while crossing a small forest trail. Other sympatric members of Pareidae in both localities include Pareas formosensis. The diet of Pareas abros sp. nov. is unknown; it likely consists of terrestrial mollusks as in other congeners.

2058

2059

2060

\section{Pareas temporalis Le, Tran, Hoang \& Stuart, 2021}

2061

Figures 6J, 20-21; Table 2; Supplementary Tables S11, S13.

2062

2063

2064

2065

2066

2067

2068

2069

2070

2071

2072

2073

Holotype: UNS 09992 (field number LD25711), adult female, Doan Ket Commune, Da Huoai District, Lam Dong Province, Vietnam $\left(11.340370^{\circ} \mathrm{N}, 107.620561^{\circ} \mathrm{E}\right.$, elevation of $496 \mathrm{~m}$ a.s.1.), coll. 25 July 2020 by Duong T.T. Le and Thinh G. Tran.

Reffered specimens ( $\mathrm{n}=6$ ): ZMMU R-13656 (field number NAP-01610), adult male collected by N. A. Poyarkov from the low-elevation disturbed bamboo forest within the valley of the Sui Lan River in the environs of Ben Cau and Phuok Son ranger stations, Cat Loc sector of the Cat Tien National Park, Lam Dong Province, southern Vietnam (N 11.69444, E 107.30639; elevation 135 m asl.) on June 20, 2011; DTU 471, adult female, collected by L. H. Nguyen and H. M. Pham from the valley of Suoi Lanh Stream, Rung Ge Commune, Di Linh District, Lam Dong Province, southern Vietnam (N 11.46725, E 108.06915; elevation of ca. 1,320 m asl.) on March 1, 2019; SIEZC 20214, adult female, collected by L. H. Nguyen in Gia Bac District, Lam Dong Province, southern Vietnam (N 14.220392, E 108.317133; elevation of ca. 1,050 m asl.) on August 10, 2018; and SIEZC 20215, adult female, collected by V. B. Tran from Biduop - Nui Ba 
2074 N.P., Lam Dong Province, southern Vietnam (N 12.23383, E 108.44866; elevation of ca. 790 m 2075 asl.) on May 30, 2017; DTU 486-487 (two adult females) collected by T. A. Pham and T. V. 2076 Nguyen in Rung Ge Commune, Di Linh District, Lam Dong Province, southern Vietnam (N 2077 11.46725, E 108.06915; elevation of ca. 1,320 m asl.), on May 10, 2020.

2078 Remark: Until our study this recently discovered species was known only from a single 2079 female specimen (Le et al., 2021). In the present work we provide morphological and genetic 2080 data for six additional specimens of this species of the both sexes from three previously unkown 2081 localities from southern Vietnam (see Fig. 1).

2082 Updated diagnosis: Pareas temporalis differs from other members of the genus Pareas by 2083 the following combination of morphological characters: body size large (TL 555-665 mm); head 2084 distinctly flattened dorsoventrally; the anterior pair of chin shields notably longer than broad; 2085 loreal and prefrontal not contacting the eye; two suboculars; generally two (rarely one or absent) 2086 postoculars; temporals generally $3+3$ (rarely $3+4$ ); three median vertebral scale rows slightly enlarged; generally 8 (rarely 7) supralabial scales; generally 8 (rarely 7) infralabials scales; 15 2088 dorsal scale rows, all of them notably keeled; 187-198 ventrals; 86-92 subcaudals, all divided; dorsum bright yellowish-brown to light-orange with distinct blackish vertebral line edged with two light yellowish paravertebral lines; vertebral spots and transverse dark bands absent (Fig. 2091 21); dorsal scales with few scattered small black spots; two very clear thin black postorbital 2092 stripes beginning from the lower and upper edges of each postorbital scale; the lower postorbital stripe as two thin parallel black lines reaching the anterior part of $8^{\text {th }} \mathrm{SL}$, not continuing to the lower jaw and chin; the left and right upper postorbital stripes contacting each other at the nuchal area forming a black ring-shaped blotch (Fig. 21A-C); belly yellowish-cream with sparse brownish dusting and irregular small spots; iris in life amber-colored to bright-orange (Fig. 21A2097 C).

Description of a male specimen (ZMMU R-13656): Adult male, specimen in a good state of preservation, body dissected longitudinally along the ventral scales (Fig. 20); body slender and notably flattened laterally; head very large, distinctly flattened dorsoventrally, clearly distinct from the thin neck (head more than three times wider than neck width near the head basis), snout blunt in dorsal and lateral views; eye very large, pupil vertical and elliptical. Hemipenis not everted.

Body size. SVL 413 mm; TaL 142 mm; TL 555 mm; TaL/TL: 0.256. 
Body scalation. Dorsal scales in 15-15-15 rows, all scales notably keeled and lack apical

2106

2107

2108

2109

2110

2111

2112

2113

2114

2115

2116

2117

2118

2119

2120

2121

2122

2123

2124

2125

2126

2127

2128

2129

2130

2131

2132

2133

2134

2135 pits; vertebral scale rows and the two adjacent rows of scales (3 medial dorsal scale rows) slightly enlarged; the outermost dorsal scale row not enlarged; ventrals 198 (+ 1 preventral), lacking lateral keels; subcaudals 98, all paired; cloacal plate single.

Head scalation. Rostral not visible from above; single nasal; two internasals, much wider than long, narrowing and slightly curving back laterally (in dorsal view), anteriorly in contact with rostral, laterally in contact with nasal and loreal, posteriorly in contact with prefrontal, not contacting preocular; two large irregular pentagonal prefrontals, much larger than internasals and with a slightly diagonal suture between them, not in contact with the eye; one subhexagonal frontal, longer than wide, smaller than parietals, with the lateral sides almost parallel to each other, slightly converging posteriorly; single preocular; single postocular present on the left side, absent on the right side of the head, semicrescent in shape, not fused with subocular; presubocular absent; two suboculars; single loreal, in contact with presubocular, prefrontal, internasal and nasal, not touching the eye; $9 / 9$ supralabials, $3^{\text {rd }}$ to $5^{\text {th }}$ or $3^{\text {rd }}$ to $7^{\text {th }}$ SL touching the subocular, none of them reaching the eye, $9^{\text {th }}$ by far the largest, elongated; $1 / 1$ supraocular; $3 / 3$ anterior temporals and 3/4 posterior temporals; 7/8 infralabials, the anterior most in contact with the opposite along the midline forming a diagonal suture between them, bordering mental, the anterior five pairs of infralabials bordering the anterior chin shields; three pairs of chin shields interlaced, no mental groove under chin and throat; the anterior chin shields relatively large, much longer than broad, followed by two pairs of chin shields that are much broader than long.

Coloration. In life, dorsal surface of the head brownish with some blackish mottling and larger spots, getting denser on frontal and prefrontals. Head with two clear thin black lateral postorbital stripes: the upper one is a well-developed slate-black line starting from postocular and running backwards to dorsal scales of the neck, the left and right upper postorbital stripes contacting each other at the nuchal area forming a black X-shaped pattern in dorsal view (Fig. 20E), and a large ring-shaped blotch in lateral view (Fig. 20C); the lower postorbital stripe is an interrupted black line starting from the lower portion of postorbital, and running ventrally and posteriorly towards lower temporals and to $8^{\text {th }}$ and $7^{\text {th }}$ supralabials and not continuing to the lower jaw and chin. Other markings on the lateral surfaces of the head include a large dark, elongated spot on the $6^{\text {th }}$ supralabial, and a thick slate-black line running from the posterior edge of the $9^{\text {th }}$ supralabial backwards to the and further on the lateral surfaces of the neck, where it 
2136 joins the ring-shaped nuchal blotch ventrally (Fig. 20C; Fig. 21A). Supralabials yellowish-white

2137 with rare tiny brown spots. Dorsal surfaces of the body bright yellowish-brown to light-orange 2138 with distinct blackish vertebral line edged with two light yellowish paravertebral lines; vertebral 2139 spots and transverse dark bands absent (Fig. 21); dorsal scales on the sides of the body with few 2140 irregularly scattered small dark spots; the ventral surfaces of the head, body and tail is yellowish2141 cream with sparse brownish dusting and irregular small spots; iris in life amber-colored to bright2142 orange; pupil black (Fig. 21A-C). In preservative: After ten years of storage in ethanol, the 2143 general coloration pattern has not changed; light brownish and yellowish tints in the coloration of 2144 dorsum, head and eyes faded becoming grayish-brown; other features of the coloration remain 2145 unchanged (Fig. 20).

2146 Variation: Measurements and scalation features of additional specimens of P. temporalis $2147(n=7)$ is presented in Supplementary Table S11. All coloration features of the additional 2148 specimens are very similar to those described for the holotype (Le et al., 2021). The holotype 2149 UNS 09992 generally agrees with the series of specimens examined by us, it has slightly higher 2150 number of supralabials (9/8 vs. 7-8) and infralabials (8/9 vs. 7-8), higher number of anterior 2151 temporals (4/5 vs. 3/3), and generally slightly lower number of posterior temporals (3/3 vs. 3-4), 2152 it also has $2 / 3$ postoculars, while in the specimens we examined generally had 2/2 postoculars 2153 and 1/0 postoculars in the single male specimen ZMMU R-13656. We observed a certain sexual 2154 dimorphism in P. temporalis: male ZMMU R-13656 has a higher number of ventrals (198 in a 2155 single male vs. 185-188 in five females), and subcaudals (92 in male vs. 86-89 in five females). 2156 Other morphological and chromatic features showed no significant variation among the examined specimens.

Updated comparisons: Pareas temporalis differs from its sister species Pareas abros sp. nov. by a higher number of ventrals (185-198 vs. 180-184), by all dorsal scale rows strongly keeled (vs. weak keels present only on 9-11 dorsal scale rows), by a higher number of enlarged vertebral scales rows ( 3 vs. 1 ), and by the absence of dark cross-bands on the body (vs. 44-56 faint dark transverse bands present). Pareas temporalis differs from $P$. berdmorei by a higher number of ventrals (185-198 vs. 164-186), by a higher number of subcaudals (86-92 vs. 63-78), by the presence of a black ring-shaped blotch on the collar (vs. absent), and by having all dorsal scale rows strongly keeled (vs. weak keels present only on 3-13 dorsal scale rows). Pareas 
2167 generally slightly higher number of subcaudals (86-92 vs. 54-96), by having all dorsal scale 2168 rows strongly keeled (vs. weak keels present on 3-11 dorsal scale rows), and by the presence of 2169 a black ring-shaped blotch on the collar (vs. absent). Pareas temporalis differs from P. nuchalis 2170 by prefrontal not contacting the eye (vs. in contact), by a slightly lower number of ventrals (1872171198 vs. 201-220), by a lower number of subcaudals (86-92 vs. 102-120), and by having all 2172 dorsal scale rows strongly keeled (vs. all dorsal scales smooth). Finally, P. temporalis differs 2173 from Pareas kuznetsovorum sp. nov. in having all dorsal scale rows strongly keeled (vs. all 2174 dorsal scales smooth), by a higher number of enlarged vertebral scales rows (3 vs. 1), and by a 2175 higher number of vetrals (185-198 vs. 167). Morphological comparisons between all species of 2176 the subgenus Pareas are detailed in Supplementary Table S13.

2177 Updated distribution: In addition to the type locality of this species, P. temporalis is also 2178 known from four localities, all in the Lam Dong Province of southern Vietnam (localities 37-40, 2179 Fig. 1). All these localities belong to the Langbian (Da Lat) Plateau - the southernmost part of 2180 the Annamite Range, well-known by its high level of endemism in herpetofauna (Bain \& Hurley, 2181 2011; Poyarkov et al., 2021). We assume that Pareas temporalis is endemic to the Langbian Plateau; it is expected to occur on middle elevations in the adjacent provinces of southern Vietnam: Binh Phuoc, Dak Nong, Dak Lak, Ninh Thuan and Binh Thuan, and also likely might inhabit the southeasternmost part of the Mondulkiri Province of Cambodia.

Etymology: The species name "temporalis" is a Latin adjective in nominative singular, meaning "temporal" and was given in reference tothe high number of temporal scales in this species (Le et al., 2021).

Recommended English name: Di Linh slug-eating snake.

Ecology notes: Pareas temporalis is a nocturnal, elusive forest-dwelling snake inhabiting mid-elevation montane evergreen tropical forests of the Langbian Plateau and its foothills; it was recorded from elevations from 135 to $1,320 \mathrm{~m}$ asl. All specimens were spotted after rain at night between 21:00 and 01:00 $\mathrm{h}$ while crawling or perching on branches of bushes, bamboo and Calamus sp. palm leafs. The holotype was found at 21:00 h on a tree branch $1.5 \mathrm{~m}$ above the ground in disturbed mixed broadleaf and bamboo forest, where it occurred in sympatry with P. margaritophorus (Le et al., 2021). Diet of $P$. temporalis is unknown, but as in other congeners, it likely consists of terrestrial mollusks. It is sympatric with a number of other Pareas species 
2198 In Di Linh District of Lam Dong Province, P. temporalis was recorded in sympatry with four 2199 species of the genus Pareas, including P. b. unicolor comb. nov., P. margaritophorus, $P$. 2200 macularius and P. formosensis. With five species of Pareas co-occurring in the same habitat, the 2201 area of Di Linh represents the center of the genus diversity in Vietnam.

2202

2203

2204

2205

2206

2207

2208 discussion.

Eberhardtia Angel, 1920: 291, by monotypy.

\section{Synonymy:}

Northpareas Wang et al., 2020: Appendix S3 (nomen nudum).

Type species: Eberhardtia tonkinensis Angel, 1920; this taxon is currently considered a junior synonym of Pareas formosensis (Van Denburgh, 1909); see Ding et al. (2020) for

Phylogenetic definition: Eberhardtia is a maximum crown-clade name referring to the clade originating with the most recent common ancestor of Pareas formosensis and Pareas monticola, and includes all extant species that share a more recent common ancestor with these taxa than with Pareas carinatus.

Diagnosis: The members of the subgenus Eberhardtia differ from the members of the subgenus Pareas by the following combination of morphological characters: frontal subhexagonal to diamond-shaped with its lateral sides converging posteriorly (Fig. 5A); anterior pair of chin shields longer than broad (Fig. 5I-J); a single thin elongated subocular; and the ultrastructure of dorsal scales not ravine-like, having pore and arc structures, with arcs connecting to each other forming characteristic lines (Wagler, 1830; Smith, 1943; Taylor, 1965; Vogel et al., 2020; He, 2009; Guo et al., 2020; our data; see Supplementary Table S14 for 2221 details).

Etymology: Angel (1920) dedicated his new genus to the collector of the single specimen of its type species, the French botanist Philippe Albert Eberhardt (1874-1942).

Distribution: Distributed in the north-eastern part of the Oriental zoogeographic region from the Eastern Himalaya to central and eastern China, islands of Hainan, Taiwan and the southern Ryukyus, southwards throughout the Indochina to the Peninsular Malaysia and Sumatra.

Content: 20 species, including P. andersonii Boulenger; P. atayal You, Poyarkov \& Lin; 
2229 P. boulengeri (Angel); P. chinensis (Barbour); P. formosensis (van Denburgh); P. geminatus

2230 Ding, Chen, Suwannapoom, Nguyen, Poyarkov \& Vogel; P. hamptoni (Boulenger); P. iwasakii

2231 (Maki); P. kaduri Bhosale, Phansalkar, Sawant, Gowande, Patel \& Mirza; P. komaii (Maki); P.

2232 macularius Theobald; P. margaritophorus (Jan); P. modestus Theobald; P. monticola (Cantor);

2233 P. niger (Pope); P. nigriceps Guo \& Deng; P. stanleyi (Boulenger); P. victorianus Vogel, 2234 Nguyen \& Poyarkov; P. vindumi Vogel; and P. xuelinensis Liu \& Rao.

2235 Recommended English name: Northern slug-eating snakes.

2236

2237 DISCUSSION

2238

2239

Phylogeny and classification of Pareinae

In this study, we present an updated multilocus phylogeny for the ancient Asian subfamily of slug-eating snakes, the Pareinae. We estimate the basal divergence within the Pareinae as the late Eocene (ca. 39.3 mya) making this group one of the oldest radiations of Colubroidea snakes (Zaher et al., 2019; Li et al., 2020). Our study includes representatives of all currently recognized taxa within of the subfamily and is, to the best of our knowledge, the most comprehensive among the published works both in terms of taxon and gene sampling. Our integrative analysis of the molecular and morphological data resolves several long-standing systematic controversies regarding the subfamily Pareinae. In particular, we confidently resolve the phylogenetic relationships among the three genera of the Pareinae: Aplopeltura is strongly suggested as the sister genus of Pareas sensu lato, while the genus Asthenodipsas is reconstructed as the most basal taxon within the subfamily with sister relationships to the clade Aplopeltura + Pareas. This topology contradicts several earlier studies on the taxonomy of the group (e. g., Guo et al., 2011; Wang et al., 2020), but generally agrees with the recent multilocus phylogenetic study by Deepak et al. (2019), though in our phylogeny we got higher values of node support.

We also provide strong evidence for the monophyly of all Pareinae genera. While monophyly of the presently monotypic genus Aplopeltura was never questioned, a number of studies suggested that the genera Pareas and Asthenodipsas might represent paraphyletic taxa (Guo et al., 2011; Pyron et al., 2011; Wang et al., 2020), or were recovered as monophyletic groups but without a significant support (e.g., Deepak et al., 2019). At the same time, we demonstrate the deep differentiation within both Pareas and Asthenodipsas, each of these genera 
2260 the early to middle Oligocene (ca. 30-31.3 mya). We also show that, though the monophyly of

2261 Pareas and Asthenodipsas is not questioned anymore, the major clades within these genera 2262 demonstrate significant differences among each other in external morphology, scale 2263 microornamentation, and biogeographic affinities; similar results were also obtained by a number 2264 of earlier studies (Grossmann \& Tillack, 2003; He, 2009; Guo et al., 2011; Guo, Wang \& 2265 Rao2020; Wang et al., 2020). We argued that the groups within Pareas and Asthenodipsas 2266 should be taxonomically recognized, what would enhance the diagnosability and clade stability 2267 of these taxa, make them more comparable units to other snake genera, restrain taxonomic 2268 vandalism, and eventually fully stabilize the taxonomy of Pareinae. Therefore, we recognize two 2269 subgenera within the genus Pareas sensu lato (Pareas sensu stricto and Eberhardtia stat. nov.), 2270 and two subgenera within the genus Asthenodipsas sensu lato (Asthenodipsas sensu stricto and Spondylodipsas subgen. nov.). Earlier studies which addressed the genus-level taxonomy of Pareas have either wrongly identified the type species of the genus (Guo et al., 2011), or have overlooked the existence of an available genus-level name for one of the clades (Eberhardtia Angel, 1920), what resulted in the creation of a nomen nudum ('Northpareas', see Wang et al., 2020). We would like to emphasize the importance of a thorough analysis of the available literature and possible synonyms prior to making a taxonomic decision in order to prevent publication of unavailable names or junior synonyms. Within the genus Pareas, our phylogenetic results support the recognition of two species groups within the subgenus Pareas sensu stricto $(P$. carinatus and $P$. nuchalis groups), and four species groups within the subgenus Eberhardtia stat. nov. (P. hamptoni, P. chinensis, P. margaritophorus, and P. monticola groups) (see Fig. 3); this taxonomy is largely concordant with the results of the previous studies (Guo et al., 2011; You, Poyarkov \& Lin, 2015; Bhosale et al., 2020; Vogel et al., 2020, 2021; Wang et al., 2020; Ding et al., 2020).

Although our understanding of the phylogenetic relationships within the Pareinae is now improved, it is still far from complete. For example, our phylogeny included only five of nine currently recognized species of Asthenodipsas; five species of the genus were described within the last decade, of which four were described based solely on morphological evidence (Quah et al., 2019, 2020, 2021). We would like to further stress herein that in this age of molecular genetics and biodiversity crises, the application of molecular methods became crucial for taxonomic practice in studies of herpetofaunal diversity in Southeast Asia (Smith et al., 2008; 
2291 Murphy et al., 2013). Not only the phylogenetic hypothesis is crucial for any comparative or 2292 biogeographic analyses, it now also became a keystone of biodiversity conservation (Shaffer et

2293

2294

2295

2296

2297

2298

2299

2300

2301

2302

2303

2304

2305

2306

2307

2308

2309

2310

2311

2312

2313

2314

2315

2316

2317

2318

2319

2320

2321

al., 2015; Chomdej et al., 2020). Phylogenetic studies on the remaining species of Asthenodipsas are required to fully resolve the taxonomy of the genus; furthermore, additional taxon and gene sampling will likely enhance the phylogenetic resolution on the level of the subfamily Pareinae and might lead to discovery of additional new lineages and species.

\section{Underestimated species diversity of Pareas in Indochina}

Though not being the most species-rich group of Asian snakes, the slug-eating snakes are widely distributed across the Southeast Asia and have a number of specialized morphological and ecological characteristics that are hypothesized to facilitate speciation. Being dietary specialists on terrestrial slugs and snails, the Pareinae occupy ecological niches inaccessible to other groups of Asian snakes, at the same time several species of the slug-eating snakes can successfully coexist with their congeners, likely due to niche partitioning and further specialization in preferred prey, dentition asymmetry, and feeding behavior (Chang et al., 2021). For example, up to three closely-related species of Pareas share same habitats in the areas of sympatry in Taiwan (You, Poyarkov \& Lin, 2015; Chang et al., 2021); we report for the first time the sympatric co-occurrence of six Pareas species in the montane forests of Lam Dong Province of southern Vietnam (P. temporalis, P. kuznetsovorum sp. nov., P. b. unicolor, P. macularius, $P$. margaritophorus, and P. formosensis), of which five species were recorded sharing the same habitat in Di Linh District. On the other hand, the sympatric co-occurrence of several often closely related species of Pareas, often makes correct species identification difficult, and may also lead to some cryptic species being overlooked.

Several recent taxonomic studies on Pareas have demonstrated that this genus has a high level of hidden and yet undescribed diversity (e.g. You, Poyarkov \& Lin, 2015; Bhosale et al., 2020; Vogel et al., 2020, 2021; Ding et al., 2020; Liu \& Rao, 2021; Yang et al., 2021). Out of 26 currently recognized species of Pareas (including those described in this work), twelve species were discovered within the last twelve years (Uetz, Freed \& Hošek, 2021), of which eleven species were described based on an integrative evidence from morphological and molecular data. At the same time, hasty taxonomic revisions may often lead to creation of unnecessary synonyms and taxonomic inflation (Isaac, Mallet \& Mace, 2004). For example, recently Wang et al. (2020)

Peer) reviewing PDF | (2021:08:64561:2:0:NEW 8 Dec 2021) 
2322 revised the taxonomy of the genus Pareas and described two new species from China: $P$. 2323 mengziensis (member of the $P$. hamptoni group) and $P$. menglaensis (member of the $P$. carinatus 2324 group). Subsequent work by Liu \& Rao (2021) noted that Wang et al. (2020) described their 2325 species without first resolving the historical taxonomic confusions of P. yunnanensis (Vogt) and $2326 P$. niger (Pope), at that time considered junior synonyms of P. chinensis (Wallach, Williams \& 2327 Boundi, 2014). Liu \& Rao (2021) further showed that P. mengziensis represents a subjective

\section{8}

2329

2330

2331

2332

2333

2334

2335

2336

2337

2338

2339

2340

2341

2342

2343

2344

2345

2346

2347

2348

2349

2350

2351

2352 junior synonym of $P$. niger, and clarified distribution and the phylogenetic placement of this species, which was also confirmed by our analyses. Furthermore, as demonstrated in our study, in their description of $P$. menglaensis, Wang et al. (2020) did not provide any comments on the distribution and existing junior synonyms of $P$. carinatus, including $P$. berdmorei, originally described from Myanmar. Herein we also analyze the distribution of phylogenetic relationships within the $P$. carinatus species group and further demonstrate that $P$. menglaensis actually represents a subjective junior synonym of $P$. berdmorei (see Results). Therefore we would like to further emphasize herein the importance of careful treatment of the available synonyms and especially of the examination of the respective type specimens in taxonomic practice. It is thus recommended that scientists, before describing a new taxon would thoroughly evaluate the available old names, the existing type specimens and / or new materials from the respective type localities. This would prevent the taxonomy from becoming confusing and the available taxa from being overlooked. We would also like to further stress herein the importance of international collaboration in resolving taxonomically confusing species complexes distributed across the international borders.

The combination of molecular and morphological data allowed this study to assess the diversity, clarify the actual geographical distribution as well as to evaluate the validity of the taxa included in the $P$. carinatus-nuchalis complex. As a result, our study revealed an unprecedented diversity within this complex, with six major lineages representing distinct species, each with significant genetic and morphological differences from the others (see Results). In our study, we consider $P$. carinatus sensu stricto distributed from the Tenasserim Range in the Peninsular Thailand and Myanmar southwards to Malayan Peninsula, Sumatra, Java, and Borneo Islands. We also revise the populations from the mainland Indochina and southern China previously referred to as $P$. carinatus or $P$. menglanensis (Wang et al., 2020), and revalidate $P$. berdmorei as a distinct species; this taxon is widely distributed across Indochina and the adjacent parts of 
2353 Yunnan and eastern Myanmar, while P. menglaensis is considered a subjective junior synonyms 2354 of this species. We also describe two new previously completely unknown species of Pareas 2355 from Vietnam, namely: Pareas kuznetsovorum sp. nov. (it belongs to $P$. carinatus species group 2356 and represents a sister species of P. berdmorei) and Pareas abros sp. nov., respectively. We 2357 provide additional information on morphological variation and distribution of the recently 2358 described $P$. temporalis. The recent discovery of the latter two species is quite unexpected since 2359 they are morphologically profoundly different from all other mainland members of the subgenus 2360 Pareas and according to our phylogeny and morphological similarities belong to P. nuchalis species group, what is also indicated by an earlier study of Le et al. (2021). It also should be noted, that our study represents the first record of $P$. nuchalis on Sumatra Island; this species has been previously considered to be restricted to Borneo. Overall, the revalidation of $P$. berdmorei along with description of Pareas abros sp. nov. and Pareas kuznetsovorum sp. nov. brings the total number of species in Pareas to 26 and the number of Pareinae species to 36.

In our study we also analyze geographic variation of morphological, chromatic and molecular characters within the wide-ranged species of the subgenus Pareas, namely $P$. carinatus and $P$. berdmorei. We report on a significant diversity within these species with two divergent, allopatric (to the best of our knowledge), and morphologically diagnosable groups revealed within $P$. carinatus, and three such groups within P. berdmorei. Should they be taxonomically recognized? The phylogenetic species concept (PSC, see Cracraft, 1983; reviewed by De Queiroz, 2007) suggests that the minimal monophyletic group on a tree should be considered a species. However, the recent progress in evolutionary phylogenomics allows revealing population genetic structure and estimate the geneflow among populations and even species in unparalleled detail (e.g., Benestan et al., 2015). This, however, often makes an accurate characterization of species boundaries within an evolutionary framework quite challenging: distinguishing between population-level genetic structure and species divergence is often problematic (Chan et al., 2020). A number of recent phylogenomic studies have demonstrated that a number of what was considered complexes of cryptic species actually represent highly admixed and structured metapopulation lineages, rather than true cryptic species (e.g., Chan et al., 2020, 2021). One of the adverse consequences of ignoring gene flow in species delimitation is the overestimation of species numbers by interpreting population structure as species divergence, thus enhancing taxonomic inflation (Chan et al., 2020). Therefore, in the 
2384 present paper, in order to assess the revealed diversity within P. carinatus and P. berdmorei we 2385 apply the subspecies concept sensu Hillis (2020) and Marshall et al. (2021), where subspecies

2386

2387

2388

2389

2390

2391

2392

2393

2394

2395

2396

2397

2398

2399

2400

2401

2402

2403

2404

2405

2406

2407

2408

2409

2410

2411

2412

2413

2414 are defined as geographically circumscribed lineages that may have been temporarily isolated in the past, but which may have since merged over broad zones of intergradation that not necessarily show the evidence of reproductive isolation between them. We recognize two subspecies within P. carinatus: P. c. carinatus (Sundaland and Malayan Peninsula south of Kra) and $P$. c. tenasserimicus ssp. nov. (Tenasserim Range north of Kra), and three subspecies within P. berdmorei: P. b. berdmorei (from eastern Mayanmar across Thailand to northern Laos, northern Vietnam and southern China), P. b. unicolor (southern Vietnam and Cambodia), and $P$. b. truongsonicus ssp. nov. (Northern Annamites in central Vietnam and Laos). Though with the data in hand we do not have any evidence of genetic admixture between these groups, it cannot be excluded that the future studies with a finer sampling might reveal a certain degree of geneflow among them. We herein prefer recognizing them as subspecies due to the overall morphological similarity of these lineages, which are mostly distinguished by coloration rather than scalation features, their presumably allopatric distribution pattern, their comparatively young evolutionary age (5.9-4.0 mya), and the historical precedent of use of the subspecies for describing diversity within these snakes (P. b. unicolor was originally described as a subspecies of P. carinatus) (see Results).

Despite the recent significant progress (Ding et al., 2020; Vogel et al., 2020, 2021;Le et al., 2021), our understanding of Pareas diversity is still incomplete. Our study revealed a high morphological variation among the examined specimens of $P$. berdmorei and $P$. carinatus; however our genetic sampling is not fully comparable to the morphological sampling. For example, our phylogenetic analysis lacked specimens of $P$. carinatus from the Greater Sunda Islands and the adjacent smaller offshore islands; many areas in the central Indochina also remained unassessed. Further field survey and taxonomic efforts both in Indochina and Sundaland will likely reveal additional lineages within the widely-distributed and insufficiently sampled species of Pareas.

\section{Historical biogeography of Pareinae}

The results of our biogeographic reconstruction suggest that the common ancestor of the Pareinae likely inhabited Sundaland (Fig. 2), while its sister group the Xylophiinae is restricted 
2415 to peninsular India (Deepak et al., 2019). The split between Pareinae and Xylophiinae is dated to 2416 happen during the middle Eocene (ca. 42.2 mya in our analysis, estimated as ca. 44.9 mya in 2417 Deepak et al., 2019), and likely reflects the ancient faunal exchange between the Indian 2418 Subcontinent and the Sundaland via a land bridge which existed during the early and middle 2419 Eocene (Ali \& Aitchison, 2008; Morley, 2018). Similar patterns were reported, for example, in 2420 Draconinae agamid lizards (Grismer et al., 2016), and Microhylinae narrow-mouth frogs (Garg $2421 \&$ Biju, 2019; Gorin et al., 2020). In particular, the assumptive vicariance between Pareinae and 2422 Xylophiinae and the distribution patterns of the two subfamilies remarkably resembles the 2423 divergence pattern between microhylid genera Micryletta (widely distributed across the 2424 Southeast Asia) and Mysticellus (restricted to southern peninsular India), which was dated as 242539.7 - 40.6 mya (Garg \& Biju, 2019). Our study thus provides further evidence for faunal exchange between the Indian Subcontinent and Sundaland during the middle Eocene.

Our results accord with the "upstream" colonization hypothesis in Pareinae. The general pattern of colonization in Pareinae is from Sundaland to the mainland Asia, this dispersal and subsequent vicariance likely happened during the early Oligocene (ca. 33.6 mya; Fig. 2). It should be noted, that though now the Sundaland is mostly represented by a number of archipelagos, in Oligocene it was connected to the mainland Southeast Asia via the Sunda shelf, which remained subaerial during the most part of Cenozoic (Cao et al., 2017; Morley, 2018). Therefore, the general direction of diversification in Pareinae was likely from the tropical continental margins of Sundaland to a nontropical Asian landmass. Starting with at least middle Eocene, Sundaland was covered with perhumid rainforests and became a major source of mainland Asian lineages for a vast number of taxa of plants and animals (see De Bruyn et al., 2014; Grismer et al., 2016, Grismer et al., in press; Morley, 2018, and references therein). Examples include the stream toad genus Ansonia (Grismer et al., 2017), the litter toads Leptobrachella (Chen et al., 2018), and the breadfruit genus Artocarpus (Williams et al., 2017). Our study is probably the first example of "upstream" colonization in Asian snakes. Further studies on the diversification patterns of other endemic Asian genera on a broad geographic scale might yield key insights into the drivers of speciation in Asia and result in a comprehensive picture of the regional source-sink dynamics between islands and continents.

Our analyses suggest that the common ancestor of the genus Pareas likely inhabited parts of the Indochinese Peninsula, Indo-Burma, and the areas which now became the Himalayas (Fig. 
2446 2). The basal split within Pareas is dated as early Oligocene (ca. 31.3 mya; Fig. 2), and 2447 temporally coincides with climatic shifts during this time. The period of late Eocene-early 2448 Oligocene transition was characterized by dramatically cool and dry climate in Southeast Asia 2449 (Zachos et al., 2001); during this time the perhumid forests contracted and fragmented (Milne \& 2450 Abbott, 2002; Bain \& Hurley, 2011; Buerki et al., 2013; Morley, 2018). These processes could 2451 potentially drive the initial diversification of Pareas through vicariance (Fig. 2). Moreover, the two major clades of Pareas (Fig. 2) are estimated to have begun diversification during the Miocene along with the significant growth of average temperature and humidity (ca. 23-12 mya; see Bain \& Hurley, 2011). These climatic changes lead to expansion of perhumid tropical forests and likely promoted the expansion and further diversification of Pareas. A similar pattern was recently reported by Chen et al. (2018) for Leptobrachella toads.

The large region of the Himalayas and Indo-Burma is suggested as the possible ancestral area for the subgenus Eberhardtia stat. nov., the most species-rich group of Pareas. The further diversification of this clade into species groups took place during the early to middle Miocene and likely took place in Himalaya and the adjacent parts of western and southern China, with subsequent dispersals to Indochina and the East Asian islands (Fig. 2). The Himalaya are now recognized as an area of exceptional diversity and endemism largely due to the uplift-driven speciation, suggesting that orogeny created conditions favoring rapid in situ diversification of resident lineages, which accelerated during the Miocene (reviewed in Xu et al., 2020). Our data suggest that geomorphological factors are also likely responsible for shaping the diversification within the subgenus Eberhardtia stat. nov. The origin and diversification of the four species groups within Eberhardtia stat. nov. temporally coincide with the rapid increase of uplifting of the Qinghai-Tibet Plateau during the Miocene (15-7 mya, An et al., 2001; Che et al., 2010), which finally gave rise to the intensification of the modern South Asian monsoon climate. This process is concidered to have accelerated the diversification of numerous Asian animal groups that share similar distributions with Eberhardtia stat. nov. (e.g., Che et al., 2010; Blair et al., 2472 2013; Gao et al., 2013; Chen et al., 2017, 2018; Xu et al., 2020). It is noteworthy that following the dispersal of Eberhardtia stat. nov. across the mainland East Asia, its members at least twice have independently colonized the East Asian islands of Taiwan and the southern Ryukyus during the late Miocene to early Pliocene, giving rise to an in situ diversification of a number of 
2477 reported in other groups of Asian herpetofauna (e.g., Yuan et al., 2016; Nguyen et al., 2020a; 2478 Yang \& Poyarkov, 2021; Gorin et al., 2020).

2479 The origin of the subgenus Pareas likely took place in what is now Western Indochina, 2480 from where, during the early to middle Miocene, it colonized Eastern Indochina leading to 2481 formation of several endemic lineages and species (Fig. 2). The Annamite or Truong Son Range, 2482 including the mountains areas of the Kon Tum-Gia Lai and Langbian plateaus, are reknown as 2483 the center of floral and faunal endemism (e.g. Averyanov et al., 2003; Bain \& Hurley, 2011; 2484 Monastyrskii \& Holloway, 2013; Poyarkov et al., 2014, 2021). According to our analyses, 2485 Eastern Indochina appears as an evolutionary hotspot for Pareas, having the high species 2486 diversity and degree of endemism, with up to six species of the genus sympatrically distributed 2487 in the montane forests of the Langbian Plateau. Among the surprising results of our study is the 2488 independent "downstream" colonization of Sundaland from Indochina during the late Miocene, which happened twice by the members of the P. carinatus and P. nuchalis species groups (Fig. 2 ). The recent discovery of two new species of the $P$. nuchalis group in mountain areas of Eastern Indochina (Pareas abros sp. nov., and P. temporalis by Le et al., 2021) is quite unexpected, and provides further evidence for faunal interchange between Eastern Indochina and Borneo (e.g., see Teynié et al., 2004). Throughout the late Cenozoic these territories were directly connected by a landbridge along the eastern edge of Sunda Shelf formed by the ancient delta joining the modern river systems of Mekong and Chao Phraya, and covered by lowland evergreen rain-forests (De Bruyn et al., 2014). However, similar biogeographic patterns are rarely reported for the herpetofauna (but see Wood et al., 2012; Geissler et al., 2015; Chen et al., 2017; Suwannapoom et al., 2018; Poyarkov et al., 2018a; Gorin et al., 2020; Grismer et al., in press), therefore additional sampling from other regions, including the different parts of the Sundaland, is needed to test this hypothesis. Overall, our study reinforces the idea that Indochina represents an indispensable hotspot for the evolution and maintenance of Southeast Asian biodiversity (De Bruyn et al., 2014).

\section{CONCLUSIONS}

In this work, we provide an updated phylogenetic hypothesis for the slug-eating snakes of 2507 the subfamily Pareinae based on mtDNA and nuDNA markers for 29 of 33 currently recognized 
2508 Pareinae species (88\%). We also included data for six lineages that have not been examined 2509 phylogenetically before our work, including the previously unknown two new species and two

2510

2511

2512

2513

2514

2515

2516

2517

2518

2519

2520

2521

2522

2523

2524

2525

2526

2527

2528

2529

2530

2531

2532

2533

2534

2535

2536 new subspecies of Pareas. Therefore, our study provides the most comprehensive taxon sampling for Pareinae published to date. This, along with morphological examination of 269 preserved specimens of Pareinae, including the available type specimens for the genus Pareas, allowed us to revise the phylogenetic relationships and taxonomy of the subfamily. Our work further highlights the importance of broad phylogenetic sampling, ground-level field surveys, and careful examination of type materials to achieve an accurate picture of phylogenetic relationships, global biodiversity, and evolutionary patterns in cryptic groups such as the Pareinae slug-eating snakes.

The present work clearly indicates a vast underestimation of diversity in the subgenus Pareas, and that the present taxonomy of the group is incomplete. Further integrative studies combining morphological and genetic analyses are essential for a better understanding of evolutionary relationships within this cryptic and taxonomically challenging radiation of Asian snakes. Overall, our study further highlights the importance of comprehensive and accurate taxonomic revisions not only for the better understanding of biodiversity and its evolution, but also for the elaboration of adequate conservation actions.

While $P$. c. carinatus and $P$. b. berdmorei are quite widely distributed taxa and their conservation status is of the least concern, the distribution of $P$. c. tenasserimicus ssp. nov., $P$. $b$. unicolor, and $P$. b. truongsonicus ssp. nov. is most likely restricted to comparatively narrow areas within the Indochina. At the same time, among the two newly described species Pareas kuznetsovorum sp. nov. is to date known only from a single specimen, while the ranges of Pareas abros sp. nov. and P. temporalis are restricted to isolated montane areas of Kon Tum Gia Lai and Lam Dong plateaus, respectively. The estimated ranges of the two new Pareas species are likely relatively small, however the actual extent of their distribution and population trends remain unknown; urgent actions are needed for careful assessment of their conservation status. We herein tentatively suggest that at present Pareas kuznetsovorum sp. nov., Pareas abros sp. nov., and P. temporalis should be categorized as Data Deficient (DD) according to the IUCN Red List criteria (2019). Further research is required to clarify the extent of their 
2537 distribution population trends, and natural history, thereby facilitating elaboration of adequate 2538 conservation actions.

2539 Our work further highlights the importance of the Indochinese region, including the 2540 territories of Vietnam, Laos, Cambodia, and Thailand, as one of the key biodiversity hotspots 2541 with high levels of herpetofaunal diversity and endemism (Bain \& Hurley, 2011; Geissler et al., 2542 2015; Duong et al., 2018; Nguyen et al., 2018, 2019, 2020b; Poyarkov et al., 2018b, 2019, 2021; 2543 Grismer et al., 2019, 2021a, 2021b; Chomdej et al., 2021; Uetz, Freed \& Hošek, 2021). This 2544 area is facing many pressures with major habitat loss by deforestation due to logging, the 2545 growing human population density and infrastructure development, agricultural extension, forest fires, and tourism development (Lang, 2001; Meyfroidt \& Lambin, 2009). Therefore, further studies are urgently needed to assess and manage the biodiversity and elaborate the adequate conservation efforts before more undescribed species are lost.

Overall, our study reinforces the idea of the global importance of Indochina as the principal evolutionary hotspot for the autochthonous herpetofaunal diversity, as well as a key area facilitating dispersals between East Asia, Indo-Burma and Sundaland. Further studies on phylogeny and the diversification patterns of different animal groups endemic to Asia on a broad geographic scale might provide key insights into the role of complex paleogeography and paleoclimate history as the drivers of speciation forming the extant Asian biodiversity.

\section{ACKNOWLEDGEMENTS}

The authors are grateful to Andrey N. Kuznetsov (JRVTTC) and Thai Van Nguyen (SVW) for supporting our study. We thank Vladislav A. Gorin, Evgeniya N. Solovyeva, Roman A. Nazarov, Sabira S. Idiatullina, The Anh Nguyen, Hieu Minh Pham, Huy Xuan Ngoc Nguyen, Bang Van Tran, Eduard A. Galoyan, and Anna B. Vassilieva for their support during the fieldwork and assistance in the lab or with data analysis. We are also grateful to Liudmila B. Salamakha for preparation of drawings for this paper. We are deeply bgrateful to Leonid A. Neimark, Indraneil Das, Guek Hock Ping aka Kurt Orion, Huy X. N. Nguyen, and Mali for his advices on Ancient and Modern Greek grammar and lexicon. Furthermore, we thank the 
2568 we visited their respective institutions: Jens Vindum and Alan Leviton (CAS); Yuezhao Wang, 2569 Xiaomao Zeng, Jiatang Li and Ermi Zhao (CIB); Ding Li (DL); Alan Resetar (FMNH); Nicolas 2570 Vidal and Annemarie Ohler (MNHN); Giuliano Doria (MNSG); Hmar Tlawmte Lalremsanga 2571 (MNZU); Colin McCarthy and Patrick Campbell (NHMUK); Silke Schweiger and Georg 2572 Gassner (NMW); Esther Dondorp, Pim Arntzen and Ronald de Ruiter (RMNH); Gunther Köhler 2573 and Linda Acker (SMF); George Zug, Kenneth Tighe and Ronald Heyer (USNM); Dennis 2574 Rödder and Wolfgang Böhme (ZFMK); Oliver Rödel and Frank Tillack (ZMB); Jakob 2575 Hallermann (ZMH); Frank Glaw and Michael Franzen (ZSM); and Valentina F. Orlova and 2576 Roman A. Nazarov (ZMMU). Alan Leviton (CAS) is thanked for inviting GV to CAS. Ding 2577 Liee, Jiatang Li and Zening Chen are thanked for their support of the work of GV in China. We 2578 want to thank Anastasio Zographos (Montmorency, France) for his expertise in classical Greek 2579 grammar. The authors thank the academic editor of this paper, Patrick David (MNHN) and an 2580 anonymous reviewer for their useful comments which improved the earlier draft of the 2581 manuscript.

2582

2583

\section{REFERENCES}

2584

2585

Ali JR, Aitchison JC. 2008. Gondwana to Asia: plate tectonics, paleogeography and the 2586

biological connectivity of the Indian subcontinent from the Middle Jurassic through latest Eocene (166-35 Ma). Earth-Science Reviews 88(3-4):145-166. DOI 10.1016/j.earscirev.2008.01.007.

An Z, Kutzbach JE, Prell WL, Porter SC. 2001. Evolution of Asian monsoons and phased uplift of the Himalaya-Tibetan Plateau since late Miocene times. Nature 411(6833):6266. DOI 10.1038/35075035.

Angel F. 1920. Sur deux Ophidiens nouveaux de la collection du Muséum. Bulletin du Muséum National d'Histoire Naturelle, 26(4):291-294 (in French).

Anonymous 2003. Declaration 44. Amendment of Article 74.7.3. Bulletin of Zoological Nomenclature 60(4):263.

Averyanov LV, Loc PK, Hiep NT, Harder DK. 2003. Phytogeographic review of Vietnam and adjacent areas of Eastern Indochina. Komarovia 3:1-83.

Bain RH, Hurley MM. 2011. A biogeographic synthesis of the amphibians and reptiles of 2598 Indochina. Bulletin of the American Museum of Natural History 360:1-138. 
Barraclough TG, Birky CW, Burt A. 2003. Diversification in sexual and asexual

2600

2601

2602

2603

2604

2605

2606

2607

2608

2609

2610

2611

2612

2613

2614

2615

2616

2617

2618

2619

2620

2621

2622

2623

2624

2625

2626

2627

2628 organisms. Evolution 57:2166-2172.

Benestan L, Gosselin T, Perrier C, Sainte-Marie B, Rochette R, Bernatchez L. 2015. RAD genotyping reveals fine-scale genetic structuring and provides powerful population assignment in a widely distributed marine species, the American lobster (Homarus americanus). Molecular Ecology 24(13):3299-3315. DOI 10.1111/mec.13245.

Bhosale H, Phansalkar P, Sawant M, Gowande G, Patel H, Mirza ZA. 2020. A new species of snail-eating snakes of the genus Pareas Wagler, 1830 (Reptilia: Serpentes) from eastern Himalayas, India. European Journal of Taxonomy 729:54-73. DOI 10.5852/ejt.2020.729.1191.

Blair C, Davy CM, Ngo A, Orlov NL, Shi HT, Lu, SQ, Gao L, Rao DQ, Murphy RW. 2013. Genealogy and demographic history of a widespread amphibian throughout Indochina. Journal of Heredity 104(1):72-85 DOI 10.1093/jhered/ess079.

Boie F. 1828. Auszüge aus Briefen von Heinr. Boie zu Java an Hn. Schlegel, Conservator anim. vertebr. am Königl. niederl. Museum. Isis von Oken, Jena, 21(10): col. 1025-1035 (in German).

Boulenger GA. 1900. Description of new reptiles and batrachians from Bomeo. Proceedings of the Zoological Society of London:182-187.

Bourret R. 1934. Notes herpétologiques sur 1'Indochine française. IV. Sur une collection d'ophidiens de Cochinchine et du Cambodge. Bulletin Général de l'Instruction Publique 14(1):5-17 (in French).

Buerki S, Forest F, Stadler T, Alvarez N. 2013. The abrupt climate change at the Eocene-Oligocene boundary and the emergence of South-East Asia triggered the spread of sapindaceous lineages. Annals of Botany 112:151-160. DOI 10.1093/aob/mct106.

Cao W, Zahirovic S, Flament N, Williams S, Golonka J, Müller RD. 2017. Improving global paleogeography since the late Paleozoic using paleobiology. Biogeosciences 14(23):54255439.

Chan KO, Hutter CR, Wood Jr PL, Grismer LL, Das I, Brown RM. 2020. Gene flow creates a mirage of cryptic species in a Southeast Asian spotted stream frog complex. Molecular Ecology 29(20):3970-3987. DOI 10.1111/mec.15603. 
Chan KO, Hutter CR, Wood PL, Su YC, Brown RM (2021). Gene flow increases

2630

2631

2632

2633

2634

2635

2636

2637

2638

2639

2640

2641

2642

2643

2644

2645

2646

2647

2648

2649

2650

2651

2652

2653

2654

2655

2656

2657

2658

phylogenetic structure and inflates cryptic species estimations: a case study on widespread Philippine puddle frogs (Occidozyga laevis). Systematic Biology, syab034. DOI 10.1093/sysbio/syab034.

Chan-ard T, Grossmann W, Gumprecht A, Schulz KD. 1999. Amphibians and reptiles of peninsular Malaysia and Thailand - an illustrated checklist [bilingual English and German]. Bushmaster Publications, Würselen, Gemany, 240 pp.

Chan-ard T, Parr JWK, Nabhitabhata J. 2015. A field guide to the Reptiles of Thailand. Oxford University Press, New York.

Chang KX, Huang BH, Luo MX, Huang CW, Wu SP, Nguyen HN, Lin SM. 2021. Niche partitioning among three snail-eating snakes revealed by dentition asymmetry and prey specialisation. Journal of Animal Ecology 90(4):967-977. DOI: 10.1111/1365-2656.13426.

Che J, Zhou W-W, Hu J-S, Yan F, Papenfuss TJ, Wake DB, Zhang Y-P. 2010. Spiny frogs (Paini) illuminate the history of the Himalayan region and southeast Asia. Proceedings of the National Academy of Sciences 107:13765-13770. DOI 10.1073/pnas.1008415107.

Chen J-M, Poyarkov NA, Suwannapoom C, Lathrop A, Wu Y-H, Zhou W-W, Yuan Z-Y, Jin J-Q, Chen H-M, Liu H-Q, Nguyen TQ, Nguyen SN, Duong TV, Eto K, Nishikawa K, Matsui M, Orlov NL, Stuart BL, Brown RM, Rowley JJL, Murphy RW, Wang Y-Y, Che J. 2018. Large-scale phylogenetic analyses provide insights into unrecognized diversity and historical biogeography of Asian leaf-litter frogs, genus Leptolalax (Anura: Megophryidae). Molecular Phylogenetics and Evolution 124:162-171. DOI 10.1016/j.ympev.2018.02.020.

Chen J-M, Zhou W-W, Poyarkov NA, Stuart BL, Brown RM, Lathrop A, Wang Y-Y, Yuan Z-Y, Jiang K, Hou M, Chen H-M, Suwannapoom C, Nguyen SN, Duong TV, Papenfuss TJ, Murphy RW, Zhang Y-P, Che J. 2017. A novel multilocus phylogenetic estimation reveals unrecognized diversity in Asian horned toads, genus Megophrys sensu lato (Anura:Megophryidae). Molecular Phylogenetics and Evolution 106:28-43. DOI 10.1016/j.ympev.2016.09.004.

Chiari Y, Vences M, Vieites DR, Rabemananjara F, Bora P, Ramilijaona Ravoahangimalala O, Meyer A. 2004. New evidence for parallel evolution of colour patterns in Malagasy poison frogs (Mantella). Molecular Ecology 13:3763-3774. 

Poyarkov NA, Che J, Gao Y-C, Gong S-P. 2021. Phylogenetic analyses of distantly related

2661

2662

2663

2664

2665

2666

2667

2668

2669

2670

2671

2672

2673

2674

2675

2676

2677

2678

2679

2680

2681

2682

2683

2684

2685

2686

2687

2688

2689

clades of bent-toed geckos (genus Cyrtodactylus) reveal an unprecedented amount of cryptic diversity in northern and western Thailand. Scientific Reports 11:2328. DOI 10.1038/s41598020-70640-8.

Chomdej S, Suwannapoom C, Pawangkhanant P, Pradit W, Nazarov RA, Grismer LL, Poyarkov NA. 2020. A new species of Cyrtodactylus Gray (Squamata:Gekkonidae) from western Thailand and the phylogenetic placement of $C$. inthanon and $C$. suthep. Zootaxa 4838(2):179-209. DOI 10.11646/zootaxa.4838.2.2.

Cochran DM. 1930. The herpetological collections made by Dr. Hugh M. Smith in Siam from 1923 to 1929. Proceedings of the United States National Museum 77(2834):1-39.

Cox MJ, Van Dijk PP, Nabhitabhata J, Kumthorn T. 1998. A photographic guide to snakes and other reptiles of Peninsular Malaysia, Singapore and Thailand. Ralph Curtis Publishing, 144 pp.

Coyne JA, Allen OH. 1998. The evolutionary genetics of speciation. Philosophical Transactions of the Royal Society B 353(1366):287-305. DOI 10.1098/rstb.1998.0210.

Cracraft J. 1983. Species concepts and speciation analysis. In: Johnston R.F. (eds). Current Ornithology, vol 1. Springer, New York, NY. DOI 10.1007/978-1-4615-6781-3_6.

Cundall D, Greene H.W. 2000. Feeding in snakes. In: Feeding: Form, Function and Evolution in Tetrapod Vertebrates (K. Schwenk, ed), pp. 293-333. Academic Press, San Diego.

Danaisawadi P, Asami T, Ota H, Sutcharit C, Pahan S. 2016. A snail-eating snake recognizes prey handedness. Scientific Reports 6:23832. DOI 10.1038/srep23832.

Danaisawadi P, Asami T, Ota H, Sutcharit C, Panha S. 2015. Subtle asymmetries in the snail-eating snake Pareas carinatus (Reptilia: Pareatidae). Journal of Ethology 33:243-246. DOI 10.1007/s10164-015-0432-x.

Das I, Dattagupta B, Gayen NC. 1998. History and catalogue of reptile types in the collection of the Zoological Survey of India. Journal of South Asian Natural History 3:121-172.

Das I. 2012. A naturalist's guide to the snakes of South-East Asia: Malaysia, Singapore, Thailand, Myanmar, Borneo, Sumatra, Java and Bali. John Beaufoy Publishing, Oxford, 176 pp.

Das I. 2018. A naturalist's guide to the snakes of Thailand and South-east Asia. 2nd edition. John Beaufoy Publishing, Oxford, 176 pp.

Peer) reviewing PDF | (2021:08:64561:2:0:NEW 8 Dec 2021) 
David P, Vogel G, Dubois A. 2011. On the need to follow rigorously the Rules of the

2691

2692

2693

2694

2695

2696

2697

2698

2699

2700

2701

2702

2703

2704

2705

2706

2707

2708

2709

2710

2711

2712

2713

2714

2715

2716

2717

2718

2719

2720

Code for the subsequent designation of a nucleospecies (type species) for a nominal genus which lacked one:the case of the nominal genus Trimeresurus Lacépède, 1804 (Reptilia: Squamata: Viperidae). Zootaxa 2992:1-51. DOI 10.11646/zootaxa.2992.1.1.

David P, Vogel G. 1996. The snakes of Sumatra, an annotated checklist and key with natural history notes. Ed. Chimaira, Frankfurt-am-Main, 260 pp.

De Bruyn M, Nugroho E, Hossain MM, Wilson JC, Mather PB. 2005. Phylogeographic evidence for the existence of an ancient biogeographic barrier: the Isthmus of Kra Seaway. Herdedity 94:370-378. DOI 10.1038/sj.hdy.6800613.

De Bruyn M, Stelbrink B, Morley RJ, Hall R, Carvalho GR, Cannon CH, Van den Bergh G, Meijaard E, Metcalfe I, Boitani L, Maiorano L, Shoup R, Von Rintelen T. 2014. Borneo and Indochina are major evolutionary hotspots for Southeast Asian biodiversity. Systematic Biology 63(6):879-901. DOI 10.1093/sysbio/syu047.

De Queiroz A, Lawson R, Lemos-Espinal JA. 2002. Phylogenetic relationships of North American garter snakes (Thamnophis) based on four mitochondrial genes: how much DNA is enough? Molecular Phylogenetics \& Evolution 22:315-329. DOI 10.1006/mpev.2001.1074.

De Queiroz K. 2007. Species concepts and species delimitation. Systematic biology 56(6):879-86. DOI 10.1080/10635150701701083.

De Queiroz K. 2020. An updated concept of subspecies resolves a dispute about the taxonomy of incompletely separated lineages. Herpetological Review 51(3):459- 461.

De Rooij NDE. 1917. The Reptiles of the Indo-Australian Archipelago. Il. Ophidia. Leiden (E. J. Brill), 334 pp.

Deepak V, Narayanan S, Das S, Rajkumar KP, Easa PS, Sreejith KA, Gower DJ. 2020. Description of a new species of Xylophis Beddome, 1878 (Serpentes: Pareidae: Xylophiinae) from the western Ghats, India. Zootaxa 4755:231-250. DOI 10.11646/zootaxa.4755.2.2.

Deepak V, Ruane S, Gower DJ. 2019. A new subfamily of fossorial colubroid snakes from the Western Ghats of peninsular India. Journal of Natural History 52:2919-2934. DOI10.1080/00222933.2018.1557756.

Deuve J. 1961. Liste annotée des Serpents du Laos. Bulletin du Muséum National d'Histoire Naturelle 1:5-32. 
2722

2723

2724

2725

2726

2727

2728

2729

2730

2731

2732

2733

2734

2735

2736

2737

2738

2739

2740

2741

2742

2743

2744

2745

2746

2747

2748

2749

2750

species of the Pareas hamptoni complex (Squamata Serpentes:Pareidae) from the Golden Triangle. Taprobanica 9:174-193.

Dowling HG, Jenner JV. 1988. Snakes of Burma: checklist of reported species \& bibliography. Smithsonian Herpetological Information Service 76:1-19.

Dowling HG. 1951. A proposed standard system of counting ventrals in snakes. British Journal of Herpetology 1:97-99.

Drummond AJ, Suchard MA, Xie D, Rambaut A. 2012. Bayesian phylogenetics with BEAUti and the BEAST 1.7. Molecular Biology and Evolution 29:1969-1973. DOI 10.1093/molbev/mss075.

Duméril AMC. 1853. Prodrome de la classifcation des reptiles ophidiens. Mémoires de l'Académie de Sciences de l'Institut de France, Paris 23, 399-536 (in French).

Duméril AMC, Bibron G, and Duméril A. H. A. 1854. Erpétologie générale, ou histoire naturelle complète des reptiles. Tomé septième, Première partie. Comprenant l'histoire des serpents non venimeux, Librairie Encyclopédique de Roret, Pari. (In French).

Duong TV, Do DT, Ngo CD, Nguyen TQ, Poyarkov NA. 2018. A new species of the genus Leptolalax (Anura:Megophryidae) from southern Vietnam. Zoological Research 39:185201. DOI 10.24272/j.issn.2095-8137.2018.009.

Figueroa A, McKelvy AD, Grismer LL, Bell CD, Lailvaux SP. 2016. A species-level phylogeny of extant snakes with description of a new colubrid subfamily and genus. PLOS ONE 11:e0161070. DOI 10.1371/journal.pone.0161070.

Filardi C, Moyle R. 2005. Single origin of a pan-Pacific bird group and upstream colonization of Australasia. Nature 438:216-219 (2005). DOI 10.1038/nature04057.

Flot JF. 2010. SeqPHASE: a web tool for interconverting PHASE input/output files and FASTA sequence alignments. Molecular ecology resources 10(1):162-166.

Fontaneto D, Herniou EA, Boschetti C, Caprioli M, Melone G, Ricci C, Barraclough TG. 2007. Independently evolving species in asexual bdelloid rotifers. PLoS Biology 5(4):e87. DOI 10.1371/journal.pbio.0050087.

Frost DR, Hillis DM. 1990. Species in concept and practice: Herpetological applications. Herpetologica 46:87-104. 
Frost DR, Kluge AG, Hillis DM. 1992. Species in contemporary herpetology: comments

2752

2753

2754

2755

2756

2757

2758

2759

2760

2761

2762

2763

2764

2765

2766

2767

2768

2769

2770

2771

2772

2773

2774

2775

2776

2777

2778

2779

2780

2781 on phylogenetic inference and taxonomy. Herpetological Review, 23:46-54.

Gao YD, Harris AJ, Zhou SD, He XJ. 2013. Evolutionary events in Lilium (including Nomocharis, Liliaceae) are temporally correlated with orogenies of the Q-T plateau and the Hengduan Mountains. Molecular Phylogenetics and Evolution 68(3):443-460. DOI 10.1016/j.ympev.2013.04.026.

Garg S, Biju SD. 2019. New microhylid frog genus from Peninsular India with Southeast Asian affinity suggests multiple Cenozoic biotic exchanges between India and Eurasia. Scientific Reports 9:1906. DOI 10.1038/s41598-018-38133-x.

Geissler P, Hartmann T, Ihlow F, Rödder D, Poyarkov NA, Nguyen TQ, Ziegler T, Böhme W. 2015. The lower Mekong: an insurmountable barrier to amphibians in southern Indochina? Biological journal of the Linnean Society 114:905-914.

Gorin VA, Scherz MD, Korost DV, Poyarkov NA. 2021. Consequences of parallel miniaturisation in Microhylinae (Anura, Microhylidae), with the description of a new genus of diminutive South East Asian frogs. Zoosystematics and Evolution 97:27-54. DOI: 10.3897/zse.97.57968.

Gorin VA, Solovyeva EN, Hasan M, Okamiya H, Karunarathna DMSS, Pawangkhanant P, de Silva A, Juthong W, Milto KD, Nguyen LT, Suwannapoom C, Haas A, Bickford DP, Das I, Poyarkov NA. 2020. A little frog leaps a long way: compounded colonizations of the Indian Subcontinent discovered in the tiny Oriental frog genus Microhyla (Amphibia: Microhylidae). PeerJ 8:e9411. DOI 10.7717/peerj.9411.

Götz M. 2002. The feeding behavior of the snail-eating snake Pareas carinatus Wagler 1830 (Squamata: Colubridae). Amphibia-Reptilia 23(4):487-493. DOI $10.1163 / 15685380260462383$.

Grismer JL, Schulte JA, Alexander A, Wagner P, Travers SL, Buehler MD, Welton LJ, Brown RM. 2016. The Eurasian invasion: phylogenomic data reveal multiple Southeast Asian origins for Indian Dragon Lizards. BMC Evolutionary Biology 16(1):43. DOI 10.1186/s12862-016-0611-6.

Grismer L, Wood PL, Poyarkov NA, Le MD, Karunarathna S, Chomdej S, Suwannapoom C, Qi S, Liu S, Che J, Quah ESH, Kraus F, Oliver PM, Riyanto A, Pauwels OSG, Grismer JL. 2021a. Karstic landscapes are foci of species diversity in the World's third- 
2782 largest vertebrate genus Cyrtodactylus Gray, 1827 (Reptilia: Squamata: Gekkonidae). Diversity 2783 13:183. DOI 10.3390/d13050183.

2784

2785

2786

2787

2788

2789

2790

2791

2792

2793

2794

2795

2796

2797

2798

2799

2800

2801

2802

2803

2804

2805

2806

2807

2808

2809

2810

2811

Grismer LL, Wood PL, Aowphol A, Cota M, Grismer MS, Murdoch ML, Aguilar CA, Grismer JL. 2017 "2016". Out of Borneo, again and again: biogeography of the stream toad genus Ansonia Stoliczka (Anura:Bufonidae) and the discovery of the first limestone cavedwelling species. Biological Journal of the Linnean Society 120:371-395. DOI 10.1111/bij.12886.

Grismer LL, Wood PL, Poyarkov NA, Le MD, Kraus F, Agarwal I, Oliver PM, Nguyen N Sang Ngoc, Nguyen TQ, Welton LJ, Stuart BL, Luu VQ, Bauer AM, Quah ESH, Chan KO, Ziegler T, Ngo HT, Aowphol A, Chomdej S, Suwannapoom C, Siler D, Anuar S, Ngo TV, Grismer JL. 2021b. Phylogenetic partitioning of the third-largest vertebrate genus in the world, Cyrtodactylus Gray, 1827 (Reptilia; Squamata; Gekkonidae) and its relevance to taxonomy and conservation. Vertebrate Zoology 71:101-154. DOI 10.3897/vz.71.e59307.

Grismer LL, Wood PL, Quah ESH, Anuar S, Poyarkov NA, Thy N, Orlov NL, Thammachoti P, Seiha H. 2019. Integrative taxonomy of the Asian skinks Sphenomorphus stellatus (Boulenger, 1900) and S. praesignis (Boulenger, 1900), with the resurrection of $S$. annamiticus (Boettger, 1901) and the description of a new species from Cambodia. Zootaxa 4683(3):381-411. DOI 10.11646/zootaxa.4683.3.4.

Grossmann W, Tillack F. 2003. On the taxonomic status of Asthenodipsas tropidonotus (Van Lidth de Jeude, 1923) and Pareas vertebralis (Boulenger, 1900) (Serpentes: Colubridae: Pareatinae). Russian Journal of Herpetology 10:175-190.

Groth JG, Barrowclough GF. 1999. Basal divergences in birds and the phylogenetic utility of the nuclear RAG-1 gene. Molecular Phylogenetics and Evolution 12:115-123. DOI10.1006/mpev.1998.0603.

Guo K, Deng X. 2009. A new species of Pareas (Serpentes: Colubridae: Pareatinae) from the Gaoligong Mountains, southwestern China. Zootaxa 2008:53-60.

Guo P, Zhao E-M. 2004. Pareas stanleyi - A record new to Sichuan, China and a Key to the Chinese species. Asiatic Herpetological Research 10:280-281.

Guo Y, Wang G, Rao D-Q. 2020. Scale microornamentation of five species of Pareas (Serpentes, Pareidae) from China. Zootaxa 4742(3):565-572. DOI 10.11646/zootaxa.4742.3.10.

PeerJ reviewing PDF | (2021:08:64561:2:0:NEW 8 Dec 2021) 
2812

2813

2814

2815

2816

2817

2818

2819

2820

2821

2822

2823

2824

2825

2826

2827

2828

2829

2830

2831

2832

2833

2834

2835

2836

2837

2838

2839

2840

2841

Guo Y, Wu Y, He S, Zhao E. 2011. Systematics and molecular phylogenetics of Asian snail-eating snakes (Pareatidae). Zootaxa, 3001:57-64.

Haas, C.P.J, de. 1950. Checklist of the snakes of the Indo-Australian Archipelago (Reptilia, Ophidia). Treubia, Bogor 20(3):511-625.

Hall R. 2012. Late Jurassic-Cenozoic reconstructions of the Indonesian region and the Indian Ocean. Tectonophysics 570:1-41. DOI 10.1016/j.tecto.2012.04.021.

Hall TA. 1999. BioEdit: a user-friendly biological sequence alignment editor and analysis program for Windows 95/98/NT. In: Nucleic acids symposium series. London: Information Retrieval Ltd c1979-c2000, 95-98.

Hauser S. 2017. On the validity of Pareas macularius Theobald, 1868 (Squamata: Pareidae) as a species distinct from Pareas margaritophorus (Jan in Bocourt, 1866). Tropical Natural History 17:147-174.

He M. (2009) Discussion on taxonomy of colubrid snakes based on scale microornamentations - Unpubl. Ph.D. thesis, Sichuan University (in Chinese).

Hennig W. 1966. Phylogenetic systematics. University of Illinois Press, Urbana, Illinois USA.

Hillis DM. 2020. The detection and naming of geographic variation within species. Herpetological Review 51:52-56. DOI10.1016/S0022-3182(70)80093-0.

Hillis DM. 2021. New and not-so-new conceptualizations of species and subspecies: A reply to the "It's species all the way down" view. Herpetological Review 52:49-50.

Hoang DT, Chernomor O, von Haeseler A, Minh BQ, Vinh LS. 2018. UFBoot2: Improving the ultrafast bootstrap approximation. Molecular Biology and Evolution 35:518-522. DOI 10.1093/molbev/msx281.

Hoso M, Asami T, Hori M. 2007. Right-handed snakes: convergent evolution of asymmetry for functional specialization. Biology Letters 3:169-172. DOI 10.1098/rsbl.2006.0600.

Hoso M, Hori M. 2006. Identification of molluscan prey from feces of Iwasaki's slug snake, Pareas iwasakii. Herpetological Review, 37:174-176.

Hoso M, Hori M. 2008. Divergent shell shape as an antipredator adaptation in tropical land snails. The American Naturalist 172:726-732. DOI 10.1086/591681. 
Hoso M, Kameda Y, Wu S.P, Asami T, Kato M, Hori M. 2010. A speciation gene for

2843

2844

2845

2846

2847

2848

2849

2850

2851

2852

2853

2854

2855

2856

2857

2858

2859

2860

2861

2862

2863

2864

2865

2866

2867

2868

2869

2870

2871

2872

left-right reversal in snails results in anti-predator adaptation. Nature Communications 1:133. DOI 10.1038/ncomms1133.

Hoso M. 2007. Oviposition and hatchling diet of a snail-eating snake Pareas iwasakii (Colubridae: Pareatinae). Current Herpetology 26:41-43. DOI 10.3105/13455834(2007)26[41:OAHDOA]2.0.CO;2.

Huelsenbeck JP, Ronquist F. 2001. MrBayes: Bayesian inference of phylogenetic trees. Bioinformatics 17(8):754-755. DOI 10.1093/bioinformatics/17.8.754.

ICZN. 1999. International Code of Zoological Nomenclature. Fourth edition. London, U.K. [available online at https://www.iczn.org/the-code/the-code-online/]: International Trust for Zoological Nomenclature.

Isaac NJ, Mallet J, Mace GM. 2004. Taxonomic inflation: its influence on macroecology and conservation. Trends in ecology \& evolution 19(9):464-9.

Iskandar DT. Colijn E. 2002“2001”. A checklist of Southeast Asian and New Guinean Reptiles. Part I. Serpentes, Biodiversity Conservation Project, Jakarta.

IUCN Standards and Petitions Committee 2019. Guidelines for Using the IUCN Red List Categories and Criteria. Ver. 14. Prepared by the Standards and Petitions Committee. http:www.iucnredlist.orgdocumentsRedListGuidelines.pdf (accessed 2021-01-30).

Jan G. 1863. Elenco sistematico degli ofidi descritti e disegnati per l'iconografia generale, A. Lombardi, Milano. 143 pp.

Jobb G. 2011. TREEFINDER: March 2011 www.treefinder.de.

Jønsson KU, Fabre FH, Ricklefs RE, Fjeldså J. 2011. Major global radiation of corvoid birds originated in the proto-Papuan archipelago. Proceedings of the National Academy of Sciences 108(6):2328-33. DOI 10.1073/pnas.1018956108/

Kaiser H, Crother BI, Kelly CM, Luiselli L, O'Shea M, Ota H, Passos P, Schleip WD, Wüster W. 2013. Best practices: in the 21st century, taxonomic decisions in herpetology are acceptable only when supported by a body of evidence and published via peer-review. Herpetological Review 44(1):8-23.

Katoh K, Misawa K, Kuma K, Miyata T. 2002. MAFFT:a novel method for rapid multiple sequence alignment based on fast Fourier transform. Nucleic Acids Research 30:30593066. DOI 10.1093/nar/gkf436. 
2873

2874

2875

2876

2877

2878

2879

2880

2881

2882

2883

2884

2885

2886

2887

2888

2889

2890

2891

2892

2893

2894

2895

2896

2897

2898

2899

2900

2901

2902

Keogh JS. 1999. Evolutionary implications of hemipenial morphology in the terrestrial Australian elapid snakes. Zoological Journal of the Linnean Society 125:239-278. DOI 10.1111/j.1096-3642.1999.tb00592.x.

Kindler C, Fritz U. 2018. Phylogeography and taxonomy of the barred grass snake (Natrix helvetica), with a discussion of the subspecies category in zoology. Vertebrate Zoology 68(3):269-281.

Knowles LL, Carstens BC. 2007. Delimiting species without monophyletic gene trees, Systematic Biology 56(6):887-895. DOI 10.1080/10635150701701091.

Kojima, Y, Fukuyama, I, Kurita, T. Hossman, Nishikawa K. 2020. Mandibular sawing in a snail-eating snake. Scientific Reports 10:12670. DOI 10.1038/s41598-020-69436-7.

Kopstein F. 1936. Herpetologische Notizen. XIII. Ueber Vipera russellii von Java. Treubia 15(3):259-264.

Kumar S, Stecher G, Tamura K. 2016. MEGA7: Molecular evolutionary genetics analysis Version 7.0 for Bigger Datasets. Molecular Biology and Evolution 33(7):1870-1874. DOI 10.1093/molbev/msw054.

Lanfear R, Calcott B, Ho SYW, Guindon S. 2012. PartitionFinder: combined selection of partitioning schemes and substitution models for phylogenetic analyses. Molecular Biology and Evolution 29(6):1695-1701. DOI 10.1093/molbev/mss020.

Lang C. 2001. Deforestation in Vietnam, Laos and Cambodia. Deforestation, environment, and sustainable development: A comparative analysis 2001:111-37.

Le DT, Nguyen SHL, Pham CT, Nguyen TQ. 2014. New records of snakes (Squamata: Serpentes) from Dien Bien Province. Journal of Biology 36:460-470. DOI 10.15625/0866$7160 / \mathrm{v} 36 \mathrm{n} 4.6175$.

Le DTT, Tran TG, Hoang HD, Stuart BL. 2021. A new species of Pareas (Squamata, Pareidae) from southern Vietnam. Vertebrate Zoology 71:439-451. DOI 10.3897/vz.71.e70438.

Li J-N, Liang D, Wang Y-Y, Guo P, Huang S, Zhang P. 2020. A large-scale systematic framework of Chinese snakes based on a unified multilocus marker system. Molecular Phylogenetics and Evolution 148:106807. DOI 10.1016/j.ympev.2020.106807.

Liu S, Rao D-Q. 2021. A new species of the genus Pareas (Squamata, Pareidae) from Yunnan, China. ZooKeys 1011:121-138. DOI 10.3897/zookeys.1011.59029. 
2904

2905

2906

2907

2908

2909

2910

2911

2912

2913

2914

2915

2916

2917

2918

2919

2920

2921

2922

2923

2924

2925

2926

2927

2928

2929

2930

2931

Cryptic speciation within Asthenodipsas vertebralis (Boulenger, 1900) (Squamata: Pareatidae), the description of a new species from Peninsular Malaysia, and the resurrection of $A$. tropidonotus (Lidth de Jude, 1923) from Sumatra: an integrative taxonomic analysis. Zootaxa 3664:505-524. DOI 10.11646/zootaxa.3664.4.5.

Malkmus R, Manthey U, Vogel G. Hoffmann P. Kosuch J. 2002. Amphibians and reptiles of Mount Kinabalu (North Borneo). A.R.G. Gantner Verlag, Rugell, 404 pp.

Malkmus R, Sauer H. 1996. Ruhestellung von Pareas nuchalis und Erstnachweis dieser Art im Nationalpark Mount Kinabalu/Malaysia. Salamandra 32(1):55-58.

Manthey U. Grossmann W. (1997), Amphibien and Reptilien Südostasiens, Natur- und Tier Verlag, Münster, 512 pp.

Marshall TL, Chambers EA, Matz MV, Hillis DM. 2021. How mitonuclear discordance and geographic variation have confounded species boundaries in a widely studied snake. Molecular Phylogenetics and Evolution, 162:107194. DOI 10.1016/j.ympev.2021.107194.

Matzke NJ. 2013. Probabilistic historical biogeography: new models for founder-event speciation, imperfect detection, and fossils allow improved accuracy and model-testing. Frontiers of Biogeography 5(4):242-248. DOI 10.21425/F5FBG19694.

Mertens, R. 1930. Die Amphibien und Reptilien der Inseln Bali, Lombok, Sumbawa und Flores. Abhandlungen der Senckenbergischen Naturforschenden Gesellschaft 42(3):117-344.

Meyfroidt P, Lambin EF. 2009. Forest transition in Vietnam and displacement of deforestation abroad. Proceedings of the National Academy of Sciences 106(38):16139-16144.

Milne RI, Abbot RJ. 2002. The origin and evolution of Tertiary relict floras. Advances in Botanical Research 38:281-314. DOI 10.1016/S0065-2296(02)38033-9.

Minh BQ, Nguyen MAT, von Haeseler A. 2013. Ultrafast approximation for phylogenetic bootstrap. Molecular Biology and Evolution 30:1188-1195. DOI 10.1093/molbev/mst024.

Monastyrskii AL, Holloway JD. 2013. The biogeography of the butterfly fauna of Vietnam with a focus on the endemic species (Lepidoptera). In: Silva-Opps M (Ed.). Current Progress in Biological Research. Rijeka, Croatia, InTech, pp. 95-123. 
Morley RJ. 2018. Assembly and division of the South and South-East Asian flora in

2933

2934

2935

2936

2937

2938

2939

2940

2941

2942

2943

2944

2945

2946

2947

2948

2949

2950

2951

2952

2953

2954

2955

2956

2957

2958

2959

2960

2961 relation to tectonics and climate change. Journal of Tropical Ecology 34(4):209-234 DOI $10.1017 / \mathrm{S} 0266467418000202$.

Mulcahy DG, Lee JL, Miller AH, Chand M, Thura MK, Zug GR. 2018. Filling the BINs of life: Report of an amphibian and reptile survey of the Tanintharyi (Tenasserim) Region of Myanmar, with DNA barcode data. ZooKeys 757:85-152. DOI 10.3897/zookeys.757.24453.

Murphy RW, Crawford AJ, Bauer AM, Che J, Donnellan SC, Fritz U, Haddad CFB, Nagy ZT, Poyarkov NA, Vences M, Wang W-Z, Zhang Y. 2013. Cold Code: the global initiative to DNA barcode amphibians and nonavian reptiles. Molecular Ecology Resources 13:161-167. DOI 10.1111/1755-0998.12050.

Nguyen HN, Tran BV, Nguyen LH, Neang T, Yushchenko PV, Poyarkov NA. 2020. A new species of Oligodon Fitzinger, 1826 from Langbian Plateau, southern Vietnam, with additional information on Oligodon annamensis Leviton, 1953 (Squamata: Colubridae). PeerJ, 8:e8332. DOI 10.7717/peerj.8332.

Nguyen LT, Poyarkov NA, Le DV, Vo BD, Phan HT, Duong TV, Murphy RW, Nguyen SN. 2018. A new species of Leptolalax (Anura: Megophryidae) from Son Tra Peninsula, central Vietnam. Zootaxa 4388:1-21. DOI 10.11646/zootaxa.4388.1.1.

Nguyen LT, Poyarkov NA, Nguyen TT, Nguyen TA, Tran VH, Gorin VA, Murphy RW, Nguyen SN. 2019. A new species of the genus Microhyla Tschudi, 1838 (Amphibia: Anura: Microhylidae) from Tay Nguyen Plateau, central Vietnam. Zootaxa 4543:549-580. DOI 10.11646/zootaxa.4543.4.4.

Nguyen LT, Schmidt HA, von Haeseler A, Minh BQ. 2015. IQ-TREE: A fast and effective stochastic algorithm for estimating maximum likelihood phylogenies. Molecular Biology and Evolution 32(1):268-274. DOI 10.1093/molbev/msu300.

Nguyen SV \& Ho CT. 1996. A checklist of reptiles and amphibians in Vietnam. Science and Technology Publishing House, Hanoi, 264 pp. (in Vietnamese).

Nguyen SV, Ho CT, Nguyen TQ. 2009. Herpetofauna of Vietnam. Frankfurt: Edition Chimaira.

Nguyen TV, Brakels P, Maury N, Sudavanh S, Pawangkhanant P, Idiiatullina S, Lorphengsy S, Inkhavilay K, Suwannapoom C, Poyarkov NA. 2020b. New herpetofaunal 
2962 observations from Laos based on photo records. Amphibian \& Reptile Conservation 14:218-249 2963 (e248).

2964 Nguyen TV, Duong TV, Luu KT, Poyarkov NA. 2020a. A new species of Kurixalus 2965 (Anura: Rhacophoridae) from northern Vietnam with comments on the biogeography of the 2966 genus. Journal of Natural History 54:195-223. DOI 10.1080/00222933.2020.1728411.

2967 Okamiya H, Sugawara H, Nagano M, Poyarkov NA. 2018. An integrative taxonomic 2968 analysis reveals a new species of lotic Hynobius salamander from Japan. PeerJ 6:e5084. DOI $296910.7717 /$ peerj.5084.

Padial JM, Miralles A, De la Riva I, Vences M. 2010. The integrative future of taxonomy. Frontiers in Zoology 7:16(2010). DOI 10.1186/1742-9994-7-16.

Peters WCH. 1864. Über neue Amphibien (Typhloscincus, Typhlops, Asthenodipsas, Ogmodon). Monatsberichte der königlich Akademie der Wissenschaften zu Berlin 1864(4):271276.

Pham AV, Nguyen TQ. 2019. New records of snakes (Reptilia: Squamata: Serpentes) from Lai Chau Province, Vietnam. VNU Journal of Science:Natural Sciences and Technology 35:1-7. DOI 10.25073/2588-1140/vnunst.4854.

Pope CH. 1935. The reptiles of China. Turtles, Crocodilians, Snakes, Lizards. Natural History of Central Asia Vol. X. The American Museum of Natural History, New York, 604 pp. 2019. Counting stripes: revision of the Lipinia vittigera complex (Reptilia, Squamata, Scincidae) with description of two new species from Indochina. Zoological Research 40(5):358-393. DOI 10.24272/j.issn.2095-8137.2019.052.

Poyarkov NA, Nguyen TV, Duong TV, Gorin VA, Yang J-H. 2018a. A new limestonedwelling species of Micryletta (Amphibia: Anura: Microhylidae) from northern Vietnam. PeerJ 6:e5771. DOI 10.7717/peerj.5771. Suwannapoom C, Orlov NL. 2021. Recent progress in taxonomic studies, biogeographic analysis and revised checklist of Amphibians in Indochina. Russian Journal of Herpetology 28:1-110. DOI 10.30906/1026-2296-2021-28-3A-1-110. 
2993 from Indochina (Amphibia: Anura: Microhylidae: Asterophryinae). Zoological Research 39:1302994 157. DOI 10.24272/j.issn.2095-8137.2018.019.

2995

2996

2997

2998

2999

3000

3001

3002

3003

3004

3005

3006

3007

3008

3009

3010

3011

3012

3013

3014

3015

3016

3017

3018

3019

3020

3021

3022

3023

Poyarkov NA, Vassilieva AB, Orlov NL, Galoyan EA, Tran DTA, Le DTT, Kretova VD, Geissler P. 2014. Taxonomy and distribution of narrow-mouth frogs of the genus Microhyla Tschudi, 1838 (Anura: Microhylidae) from Vietnam with descriptions of five new species. Russian Journal of Herpetology 21:89-148.

Pyron RA, Burbrink FT, Colli GR, de Oca ANM, Vitt LJ, Kuczynski CA, Wiens JJ. 2011. The phylogeny of advanced snakes (Colubroidea), with discovery of a new subfamily and comparison of support methods for likelihood trees. Molecular Phylogenetics and Evolution 58:329-342. DOI 10.1016/j.ympev.2010.11.006.

Pyron RA, Burbrink FT, Wiens JJ. 2013. A phylogeny and revised classification of Squamata, including 4161 species of lizards and snakes. BMC Evolutionary Biology 13:93. DOI 10.1186/1471-2148-13-93.

Quah ESH, Grismer LL, Lim KKP, Anuar M. S. S, Chan KO. 2020. A taxonomic revision of Asthenodipsas malaccana Peters, 1864 (Squamata: Pareidae) with a description of a new species from Borneo. Zootaxa 4729:001-024. DOI 10.11646/zootaxa.4729.1.1.

Quah ESH, Grismer LL, Lim KKP, Anuar M. S. S, Imbun AY. 2019. A taxonomic reappraisal of the Smooth slug snake Asthenodipsas laevis (Boie, 1827) (Squamata: Pareidae) in Borneo with the description of two new species. Zootaxa 4646:501-526. DOI 10.11646/zootaxa.4646.3.4.

Quah ESH, Lim KKP, Grismer LL. 2021. On the taxonomic status of Asthenodipsas vertebralis (Boulenger, 1900) (Squamata: Pareidae) in Borneo with the description of a new species. Zootaxa 4949:24-44. DOI 10.11646/zootaxa.4949.1.2.

Rambaut A, Drummond AJ. 2007. Tracer. Version 1.5. Available at http://beast.bio.ed.ac.uk/Tracer.

Rao D-Q, Yang D-T. 1992. Phylogenetic systematics of Pareatinae (Serpentes) of Southeastern Asia and adjacent islands with relationship between it and the geology changes. Acta Zoologica Sinica 38:139-150 (in Chinese).

Ree RH, Moore BR, Webb CO, Donoghue MJ. 2005. A likelihood framework for inferring the evolution of geographic range on phylogenetic trees. Evolution 59(11):2299-2311. DOI 10.1111/j.0014-3820.2005.tb00940.x.

Peer) reviewing PDF | (2021:08:64561:2:0:NEW 8 Dec 2021) 
3024

3025

3026

3027

3028

3029

3030

3031

3032

3033

3034

3035

3036

3037

3038

3039

3040

3041

3042

3043

3044

3045

3046

3047

3048

3049

3050

3051

3052

3053

3054

Ree RH, Smith SA. 2008. Maximum likelihood inference of geographic range evolution by dispersal, local extinction, and cladogenesis. Systematic Biology 57(1):4-14. DOI $10.1080 / 10635150701883881$.

Ronquist F, Huelsenbeck JP. 2003. MrBayes 3: Bayesian phylogenetic inference under mixed models. Bioinformatics 19(12):1572-1574. DOI 10.1093/bioinformatics/btg180.

Ronquist F. 1997. Dispersal-vicariance analysis: a new approach to the quantification of historical biogeography. Systematic Biology 46(1):195-203 DOI 10.1093/sysbio/46.1.195.

Russell DW, Sambrook J. 2001. Molecular cloning: a laboratory manual. Cold Spring Harbor:Cold Spring Harbor Laboratory.

Salvi D, Harris DJ, Kaliontzopoulou A, Carretero MA, Pinho C. 2013. Persistence across Pleistocene ice ages in Mediterranean and extra-Mediterranean refugia: Phylogeographic insights from the common wall lizard. BMC Evolutionary Biology 13:147. DOI 10.1186/14712148-13-147.

Schlegel H. 1826. Notice sur l'Erpétologie de l'ile de Java; par M. Böié (Ouvrage manuscrit). Bulletin des Sciences Naturelles et de Géologie (Section II du Bulletin Universel des Sciences naturelles de Férussac) 9(2):233-240.

Schlegel H. 1837. Essai sur la physionomie des serpens. I. Partie générale. II. Partie descriptive. Atlas, 21 planches et 3 cartes. Arnz Arnz \& Comp, Leiden, 606 pp.

Schmidt D, Kunz K. 2005. Ernährung von Schlangen. Natur und Tier Verlag, Münster, $159 \mathrm{pp}$ (in German).

Shaffer, H.B, Gidis, M, McCartney-Melstad, E, Neal, K.M, Oyamaguchi, H.M, Tellez, M. \& Toffelmeir, E.M. 2015. Conservation genetics and genomics of amphibians and reptiles. Annual Review of Animal Biosciensces 3:23.1-23.26. DOI 10.1146/annurev-animal-022114110920.

Shimodaira H. 2002. An approximately unbiased test of phylogenetic tree selection. Systematic Biology 51(3):492-508.

Slowinski JB, Lawson R. 2002. Snake phylogeny: evidence from nuclear and mitochondrial genes. Molecular Phylogenetics \& Evolution 24(2):194-202. DOI 10.1016/S10557903(02)00239-7.

Smedley N. 1931. Amphibiens and Reptiles from the Cameron Highlands, Malay Peninsular. The Raffles Bulletin of Zoology 6:105-123. 
3055

3056

3057

3058

3059

3060

3061

3062

3063

3064

3065

3066

3067

3068

3069

3070

3071

3072

3073

3074

3075

3076

3077

3078

3079

3080

3081

3082

3083

3084

Smith AM, Poyarkov NA, Hebert PDN. 2008. CO1 DNA barcoding amphibians:take the chance, meet the challenge. Molecular Ecology Notes 8:235-246. DOI 10.1146/annurev-animal022114-110920.

Smith MA. 1943. The fauna of British India Ceylon and Burma, including the whole of the Indo-Chinese Sub-region. Reptilia and Amphibia. Vol. III. Serpentes. Taylor and Francis, London, 583 pp.

Stephens M, Smith NJ, Donnelly P. 2001. A new statistical method for haplotype reconstruction from population data. The American Journal of Human Genetics 68(4):978-989. DOI $10.1086 / 319501$.

Stuebing RB, Inger RF, Lardner B. 2014. A field guide to the snakes of Borneo (2nd edition). Natural History Publications (Borneo), Kota Kinabalu, 310 pp.

Suntrarachun S, Chanhome L, Hauser S, Sumontha M, Kanya K. 2020. Molecular phylogenetic support to the resurrection of Pareas macularius from the synonymy of Pareas margaritophorus (Squamata: Pareidae). Tropical Natural History 20:182-190.

Suwannapoom C, Sumontha M, Tunprasert J, Ruangsuwan T, Pawangkhanant P, Korost DV, Poyarkov NA. 2018. A striking new genus and species of cave-dwelling frog (Amphibia: Anura: Microhylidae: Asterophryinae) from Thailand. PeerJ 6:e4422. DOI 10.7717 peerj.4422.

Taylor EH. 1965. The serpents of Thailand and adjacent waters. University of Kansas Science Bulletin 45(9):609-1096.

Teynié A, David P. 2010. Voyages naturalistes au Laos. Les Reptiles. Editions Revoir, Nohanent (France), 315 pp.

Teynié A, David P, Ohler, Luanglath, K. 2004. Note on a collection of Amphibians and Reptiles from South Laos, with a discussion of the occurrence of Indo-Malayan species. Hamadryad 29(1):33-62.

Theobald W. 1868a. Catalogue of the Reptiles of British Birma, embracing the Provinces of Pegu, Martaban, and Tenasserim; with descriptions of new or little-known species. Journal of the Linnean Society, London, (Zoology), 10(1):4-67.

Theobald W. 1868b. Catalogue of Reptiles in the Museum of the Asiatic Society of Bengal. Journal of the Asiatic Society of Bengal (Extra number) 37: vi + $88+$ iii pp, 4 pls. 
Trifnopoulos J, Nguyen TL, von Haeseler A, Bui MQ. 2016. W-IQTREE: a fast online

3086

3087

3088

3089

3090

3091

3092

3093

3094

3095

3096

3097

3098

3099

3100

3101

3102

3103

3104

3105

3106

3107

3108

3109

3110

3111

3112

3113

3114

phylogenetic tool for maximum likelihood analysis. Nucleic Acids Research 44(W1):W232W235. DOI 10.1093/nar/gkw256.

Uetz P, Freed P, Hošek J. 2021. The Reptile Database, http:reptiledatabase.reptarium.cz (accessed in March 2021).

Vences M, Guayasamin JM, Miralles A, de la Riva I. 2013. To name or not to name: Criteria to promote economy of change in Linnaean classifcation schemes. Zootaxa 3636(2):201-244. DOI 10.11646/zootaxa.3636.2.1.

Vogel G, Nguyen TV, Lalremsanga HT, Biakzuala L, Hrima V, Poyarkov NA. 2020. Taxonomic reassessment of the Pareas margaritophorus-macularius species complex (Squamata, Pareidae). Vertebrate Zoology 70:547-569. DOI 10.26049/VZ70-4-2020-02.

Vogel G, Nguyen TV, Than Zaw, Poyarkov NA. 2021. A new species of the Pareas monticola complex (Squamata, Serpentes, Pareidae) from Chin Mountains with additions to the Pareas fauna of Myanmar. Journal of Natural History 54(39-40):2577-2612. DOI $10.1080 / 00222933.2020 .1856953$.

Vogel G. 2015. New montane species of the genus Pareas Wagler, 1830 (Squamata: Pareatidae) from Northern Myanmar. Taprobanica 7(1):1-7.

Wagler JG. 1830. Natürliches System der Amphibien, mit vorangehender Classification der Säugetiere und Vögel. Ein Beitrag zur vergleichenden Zoologie. 1.0. Cotta, München, Stuttgart, and Tübingen, $354 \mathrm{pp}$.

Wallach V, Wuester W, Broadley DG. 2009. In praise of subgenera: taxonomic status of cobras of the genus Naja Laurenti (Serpentes: Elapidae). Zootaxa, 2236(1):26-36.

Wallach V, Williams KL, Boundy J. 2014. Snakes of the world: a catalogue of living and extinct species. CRC press, Boca Raton:1237pp.

Wang P, Che J, Liu Q, Li K, Jin JQ, Jiang K, Shi L, Guo P. 2020. A revised taxonomy of Asia snail-eating snakes Pareas (Squamata, Pareidae): evidence from morphological comparison and molecular phylogeny. ZooKeys 939:45-64. DOI 10.3897/zookeys.939.49309.

Wilcox TP, Zwickl DJ, Heath TA, Hillis DM. 2002. Phylogenetic relationships of the Dwarf Boas and a comparison of Bayesian and bootstrap measures of phylogenetic support. Molecular Phylogenetics and Evolution 25(2):361-371. DOI 10.1016/S1055-7903(02)00244-0. 
Out of Borneo: biogeography, phylogeny and divergence date estimates of Artocarpus

(Moraceae). Annals of Botany 119:611-627. DOI 10.1093/aob/mcw249.

Wilson EO, Brown WL. 1953. The subspecies concept and its taxonomic application.

3119

3120

3121

3122

3123

3124

3125

3126

3127

3128

3129

3130

3131

3132

3133

3134

3135

3136

3137

3138

3139

3140

3141

3142

3143

3144

3145

Systematic Zoology 2(3):967-111. DOI 10.2307/2411818.

Wood PL, Guo X-G, Travers SL, Su Y-C, Olson KV, Bauer AM, Grismer LL, Siler

CD, Moyle RG, Andersen MJ, Brown RM. 2020. Parachute geckos free fall into synonymy: Gekko phylogeny, and a new subgeneric classification, inferred from thousands of ultraconserved elements. Molecular Biology and Evolution 146:106731. DOI 10.1016/j.ympev.2020.106731.

Wood PL, Heinicke MP, Jackman TR, Bauer AM. 2012. Phylogeny of bent-toed geckos (Cyrtodactylus) reveals a west to east pattern of diversification. Molecular Phylogenetics and Evolution 65(3):992-1003. DOI 10.1016/j.ympev.2012.08.025.

Wüster W, Scott T, O'Shea M, Kaiser H. 2021. Confronting taxonomic vandalism in biology: conscientious community self-organization can preserve nomenclatural stability. Biological Journal of the Linnean Society 133(3):645-670. DOI 10.1093/biolinnean/blab009.

Xu W, Dong WJ, Fu TT, Gao W, Lu CQ, Yan F, Wu YH, Jiang K, Jin JQ, Chen HM, Zhang YP. 2020. Herpetological phylogeographic analyses support a Miocene focal point of Himalayan uplift and biological diversification. National Science Review: nwaa263. DOI 10.1093/nsr/nwaa263/5934401.

Yang D-T, Rao D-Q. 2008. Amphibia and Reptilia of Yunnan, Kunming, Yunnan Publishing Group Corporation, Yunnan Science (in Chinese), 411 pp.

Yang J-H, Poyarkov NA. 2021. A new species of the genus Micryletta (Anura, Microhylidae) from Hainan Island, China. Zoological Research 42:234-240. DOI 10.24272/j.issn.2095-8137.2020.333.

Yang J-H, Yeung HY, Huang X-Y, Yang S-P. 2021. First record of Pareas vindumi Vogel, 2015 (Reptilia: Pareidae) from China with a revision to morphology. Taprobanica 10:3946. DOI 10.47605/tapro.v10i1.246.

Yang Z. 2007. PAML 4: phylogenetic analysis by maximum likelihood. Molecular Biology and Evolution 24(8):1586-1591. DOI10.1093/molbev/msm088.

You CW, Poyarkov NA, Lin SM. 2015. Diversity of the snail-eating snakes Pareas (Serpentes, Pareatidae) from Taiwan. Zoologica Scripta 44(4):349-361. DOI 10.1111/zsc.12111. 
Yu Y, Harris AJ, Blair C, He X. 2015. RASP (Reconstruct Ancestral State in 3147 Phylogenies): a tool for historical biogeography. Molecular Phylogenetics and Evolution 87:463148 49 DOI 10.1016/j.ympev.2015.03.008.

Yuan ZY, Zhou WW, Chen X, Poyarkov NA, Chen HM, Jang-Liaw NL, Chou WH, Matzke NJ, Iizuka K, Min MS, Kuzmin SL, Zhang YP, Cannatella DC, Hillis DM, Che J. 3151 2016. Spatiotemporal diversification of the true frogs (genus Rana): an historical framework for 3152 a widely studied group of model organisms. Systematic Biology 65(5):824-842; DOI10.1093/sysbio/syw055.

Zachos J, Pagani M, Sloan L, Thomas E, Billups K. 2001. Trends, rhythms, and 3156 aberrations in global climate $65 \mathrm{Ma}$ to present. Science 292:686-693. DOI 10.1126/science.1059412.

Zaher H, Murphy RW, Arredondo JC, Graboski R, Machado-Filho PR, Mahlow K, Montingelli GG, Quadros AB, Orlov NL, Wilkinson M, Zhang Y-P, Grazziotin FG. 2019. Large-scale molecular phylogeny, morphology, divergence-time estimation, and the fossil record of advanced caenophidian snakes (Squamata: Serpentes). PLoS ONE 14:e0216148. DOI 10.1371/journal.pone.0216148.

Ziegler T, Ohler A, Vu NT, Le KQ, Nguyen XT, Dinh HT, Bui NT. 2006. Review of the amphibian and reptile diversity of Phong Nha - Ke Bang National Park and adjacent areas, central Truong Son, Vietnam. In: Vences M, Köhler J, Ziegler T, Böhme W (Eds.): Herpetologia Herpetologica, Bonn: 247-262. 


\section{Table 1 (on next page)}

Table 1. Species-level scientific names erected for the members of the subgenus Pareas. 
1 Table 1. Species-level scientific names erected for the members of the subgenus Pareas.

\begin{tabular}{|c|c|c|c|c|c|}
\hline No. & Authority & Original taxon name & Type locality & Previous taxonomy & New taxonomy \\
\hline 1 & Wagler, 1830 & Pareas carinata & Java, Indonesia & Pareas carinatus & Pareas carinatus \\
\hline 2 & Theobald, 1868 & Pareas berdmorei & Mon State, Myanmar & synonym of Pareas carinatus & Pareas berdmorei \\
\hline 3 & Boulenger, 1900 & Amblycephalus nuchalis & Matang, Kidi District, Sarawak, Malaysia & Pareas nuchalis & Pareas nuchalis \\
\hline 4 & Bourret, 1934 & Amblycephalus carinatus unicolor & Kampong Speu Province, Cambodia & synonym of Pareas carinatus & Pareas berdmorei unicolor comb. nov. \\
\hline 5 & Wang et al., 2020 & Pareas menglaensis & Mengla County, Yunnan Province, China & Pareas menglaensis & synonym of Pareas berdmorei \\
\hline 6 & Le et al., 2021 & Pareas temporalis & $\begin{array}{l}\text { Doan Ket Commune, Da Huoai District, Lam } \\
\text { Dong Province, Vietnam }\end{array}$ & Pareas temporalis & Pareas temporalis \\
\hline 7 & this paper & Pareas carinatus tenasserimicus & $\begin{array}{l}\text { Suan Phueng District, Ratchaburi Province, } \\
\text { Thailand }\end{array}$ & - & Pareas carinatus tenasserimicus ssp. nov. \\
\hline 8 & this paper & Pareas berdmorei annamiticus & Nahin District, Khammouan Province, Laos & - & Pareas berdmorei annamiticus ssp. nov. \\
\hline 9 & this paper & Pareas kuznetsovorum & $\begin{array}{l}\text { Song Hinh District, Phu Yen Province, } \\
\text { Vietnam }\end{array}$ & - & Pareas kuznetsovorum sp. nov. \\
\hline 10 & this paper & Pareas abros & $\begin{array}{l}\text { Song Thanh N.P., Quang Nam Province, } \\
\text { Vietnam }\end{array}$ & - & Pareas abros sp. nov. \\
\hline
\end{tabular}




\section{Table 2 (on next page)}

Table 2. Measurements and meristic characters of members of the subgenus Pareas:

Pareas abros sp. nov., P. kuznetsovorum sp. nov., P. carinatus, $P$. berdmorei, $P$. nuchalis and $P$. temporalis.

Abbreviations are listed in the Materials and methods. 
Table 2. Measurements and meristic characters of members of the subgenus Pareas: Pareas abros sp. nov., P. kuznetsovorum sp. nov., P. carinatus, $P$. berdmorei, $P$. nuchalis and $P$. temporalis.

\begin{tabular}{|c|c|c|c|c|c|c|c|}
\hline \multicolumn{2}{|c|}{ Characters } & P. abros & P. kuznetsovorum & P. carinatus & P. berdmorei & P. nuchalis & P. temporali \\
\hline \multirow{3}{*}{ TL } & Min-Max & $434-565$ & \multirow{2}{*}{638.5} & $337-702$ & $421-770$ & $555-665$ & $555-665$ \\
\hline & Mean \pm SD & $506.7 \pm 66.7$ & & $494.3 \pm 73.3$ & $554.9 \pm 73.3$ & $577.1 \pm 34.5$ & $577.1 \pm 34.5$ \\
\hline & $\mathrm{n}$ & 3 & 1 & 24 & 34 & 7 & 7 \\
\hline \multirow{3}{*}{ TaL/TL } & Min-Max & $0.26-0.29$ & \multirow{2}{*}{0.25} & $0.18-0.25$ & $0.17-0.27$ & $0.20-0.26$ & $0.20-0.26$ \\
\hline & Mean \pm SD & $0.28 \pm 0.01$ & & $0.22 \pm 0.02$ & $0.21 \pm 0.02$ & $0.24 \pm 0.02$ & $0.24 \pm 0.02$ \\
\hline & $\mathrm{n}$ & 3 & 1 & 24 & 32 & 7 & 7 \\
\hline \multirow{3}{*}{ VEN } & Min-Max & $180-184$ & \multirow{2}{*}{167} & $158-194$ & $162-187$ & $185-198$ & $185-198$ \\
\hline & Mean \pm SD & $182.7 \pm 2.3$ & & $171.4 \pm 9.3$ & $176.9 \pm 5.8$ & $189.0 \pm 4.4$ & $189.0 \pm 4.4$ \\
\hline & $\mathrm{n}$ & 3 & 1 & 26 & 38 & 7 & 7 \\
\hline \multirow{3}{*}{ SC } & Min-Max & $83-95$ & \multirow{2}{*}{87} & $54-96$ & $57-89$ & $86-92$ & $86-92$ \\
\hline & Mean \pm SD & $90.0 \pm 6.2$ & & $69.3 \pm 9.0$ & $71.6 \pm 7.3$ & $88.7 \pm 2.4$ & $88.7 \pm 2.4$ \\
\hline & $\mathrm{n}$ & 3 & 1 & 26 & 34 & 7 & 7 \\
\hline \multirow{3}{*}{ KMD } & Min-Max & $9-11$ & \multirow{2}{*}{0} & $0-11$ & $3-13$ & 15 & 15 \\
\hline & Mean \pm SD & $10.3 \pm 1.1$ & & $6.5 \pm 2.9$ & $8.83 \pm 2.76$ & $15.0 \pm 0.0$ & $15.0 \pm 0.0$ \\
\hline & $\mathrm{n}$ & 3 & 1 & 19 & 33 & 7 & 7 \\
\hline \multirow{3}{*}{ VSC } & Min-Max & 1 & \multirow{2}{*}{1} & 3 & $1-3$ & 3 & 3 \\
\hline & Mean \pm SD & $1.0 \pm 0.0$ & & $3.0 \pm 0.0$ & $2.83 \pm 0.56$ & $3.0 \pm 0.0$ & $3.0 \pm 0.0$ \\
\hline & $\mathrm{n}$ & 3 & 1 & 26 & 38 & 7 & 7 \\
\hline
\end{tabular}




\section{Figure 1}

Figure 1. Map showing distribution of the subgenus Pareas and location of studied populations.

Circles denote localities for which both DNA and morphological data were examined;

triangles denote populations for which only DNA data were available; filled diamonds denote localities for which only morphological data were available; empty diamonds denote localities for which only photo data was available and therefore the species identification is tentative (a-c); dot in the center of an icon indicates the type locality. Confirmed localities: (1) Indonesia, Java; (2) Indonesia, West Java, Bogor; (3) Indonesia, West Java; (4) Indonesia, Central Java, Karimundjava Isl.; (5) Indonesia, East Java; (6) Indonesia, Sumatra, Ranau Lake; (7) Indonesia, North Sumatra; (8) Indonesia, Borneo, Central Kalimantan, Moara Terweh; (9) Indonesia, Borneo, East Kalimantan, Kutai N.P.; (10) Malaysia, Borneo, Sarawak; (11) Malaysia, Pahang, Frazers Hills; (12) Malaysia, Terengganu; (13) Malaysia, Kedah, Sungai Sedim; (14) Thailand, Ratchaburi, Suan Phueng; (15) Myanmar, Tanintharyi, Yaephyu; (16) Myanmar, Mon; (17) Myanmar, Mon, Kyaikhto, Kinpon Chaung; (18) Thailand, Chiang Mai, Doi Inthanon N.P.; (19) Thailand, Phitsanulok, Phu Hin Rong Kla N.P.; (20) Laos, Luangphrabang; (21) Laos, Phongsaly; (22) China, Yunnan, Mengla; (23) China, Yunnan, Xishuangbannna; (24) China, Yunnan, Pu'er; (25) Vietnam, Dien Bien, Muong Nhe N.R.; (26) Vietnam, Vinh Phuc, Tam Dao N.P.; (27) Laos, Khammouan, Nahin; (28) Vietnam, Quang Binh, Thanh Thach; (29) Cambodia, Kampong Speu; (30) Vietnam, An Giang, Bay Nui Mt.; (31) Vietnam, Dong Nai, Trang Bom; (32) Vietnam, Dong Nai, Ma Da N.R. (Vinh Cuu); (33) Vietnam, Dong Nai, Cat Tien N.P.; (34) Vietnam, Tay Ninh, Lo Go - Xa Mat N.P.; (35) Vietnam, Binh Phuoc, Bu Gia Map N.P.; (36) Vietnam, Lam Dong, Loc Bao; (37) Vietnam, Lam Dong, Di Linh; (38) Vietnam, Lam Dong, Cat Loc; (39) Vietnam, Lam Dong, Da Huoai; (40) Vietnam, Lam Dong, Bidoup - Nui Ba N.P.; (41) Vietnam, Phu Yen, Song Hinh; (42) Vietnam, Quang Nam, Song Thanh N.P.; (43) 
Vietnam, Thua Thien-Hue, A Roang, Sao La N.R.; (44) Malaysia, Sarawak, Betong, Saribas; (45) Malaysia, Sarawak, Niah N.P.; (46) Malaysia, Sarawak, Bintulu; (47) Brunei, Brunei Darussalam; (48) Malaysia, Sabah, Tenom; (49) Indonesia, Sumatra, Riau, Indragiri; (50) Indonesia, Sumatra, Bengkulu, Kepahiang. Unconfirmed Iocalities: (a) Thailand, Phetchaburi, Kaeng Krachan N.P.; (b) Laos, Champasak, Xe Pian N.P.A.; (c) Vietnam, Quang Binh, Phong Nha - Ke Bang N.P.. Base Map created using simplemappr.net. 


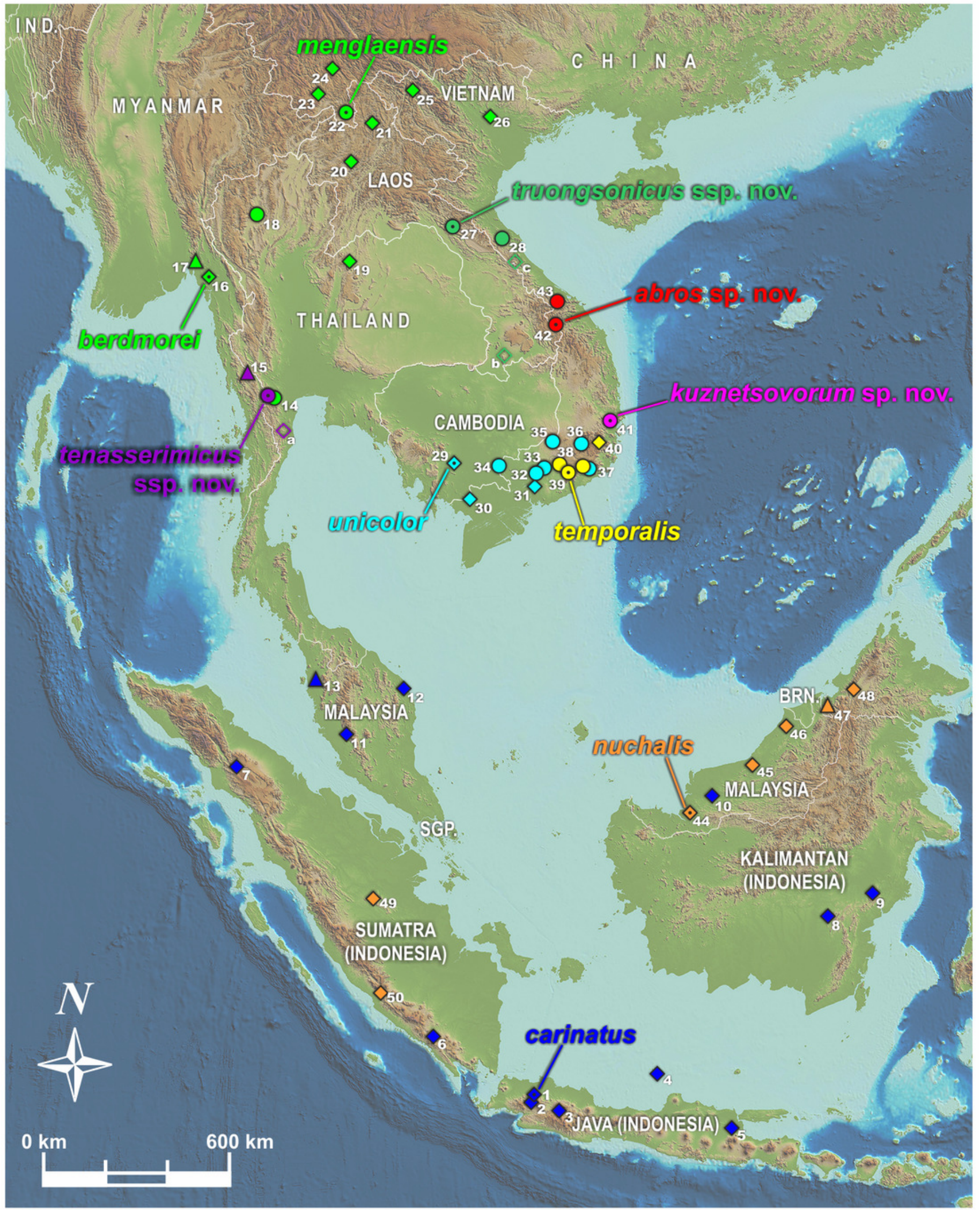




\section{Figure 2}

Figure 2. Time tree and biogeographic history of the subfamily Pareinae.

(A) Biogeographic regions used in the present study; (B) BEAST chronogram on the base of $3588 \mathrm{bp}$-long mtDNA + nuDNA dataset with the results of ancestral area reconstruction using Langrange Dispersal-Extinction-Cladogenesis (DEC) model in RASP. For biogeographic areas definitions, species occurrence data and transition matrices see Supplementary Tables S5 and S6. Information at tree tips corresponds to biogeographic area code (see Fig. 2A), sample number (summarized in Supplementary Table S1), and species name, respectively. Node colors correspond to the respective biogeographic areas; values inside node icons correspond to node numbers (see Supplementary Table S9 and Supplementary Figure S1 for divergence time estimates); values in grey near nodes indicate marginal probabilities for ancestral ranges (S-DIVA analysis), values in blue near nodes correspond to median time of divergence (see Supplementary Table S9); icons illustrate vicariant and dispersal events (see Legend). Base Map created using simplemappr.net. 


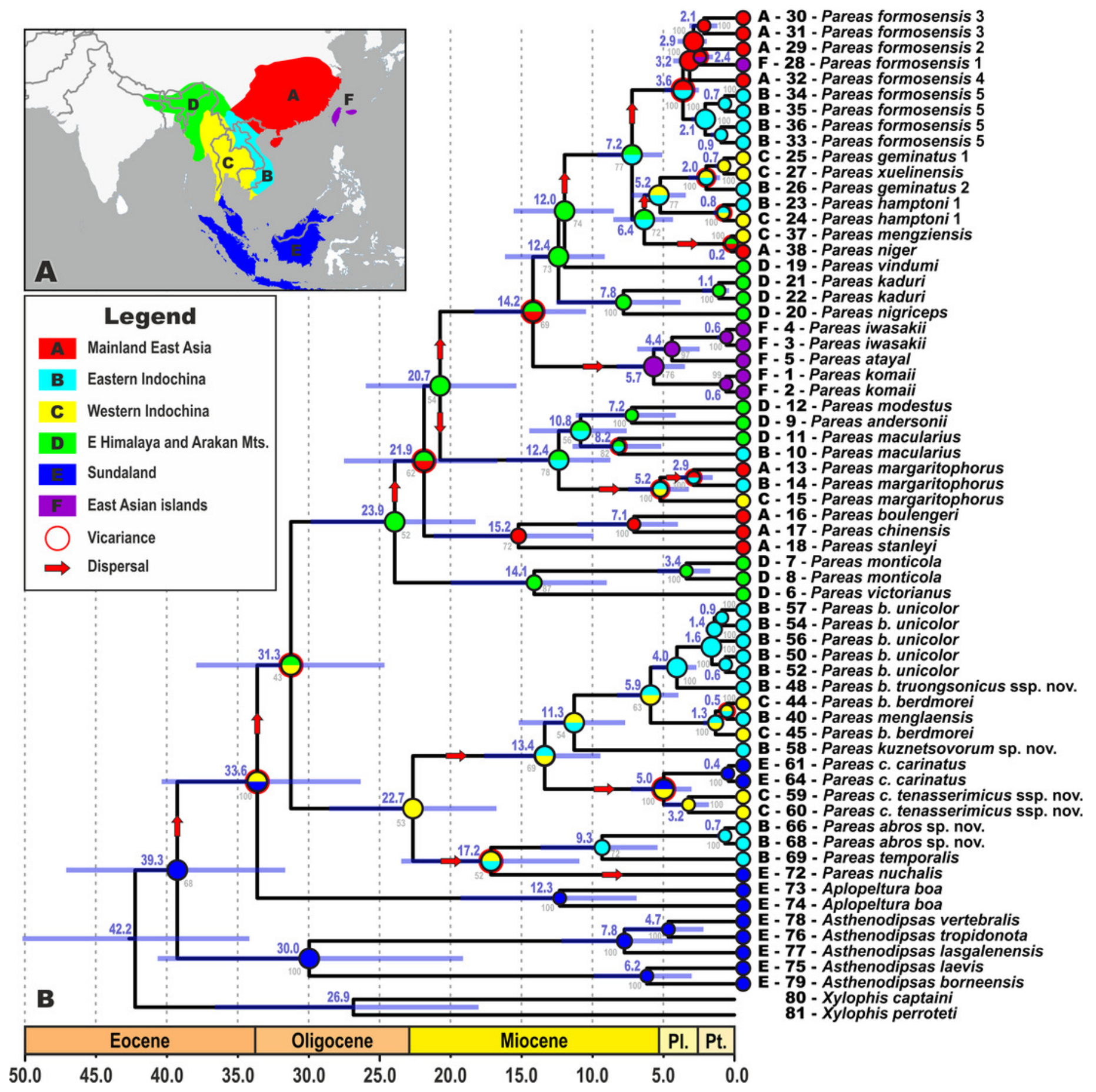




\section{Figure 3}

Figure 3. Bayesian inference tree of the subfamily Pareinae derived from the analysis of 1,126 bp of cyt b, $681 \mathrm{bp}$ of ND4, $737 \mathrm{bp}$ of cmos, and 1,026 bp of RAG1 gene fragments.

For voucher specimen information and GenBank accession numbers see Supplementary Table S1. Colors denote the taxa of the subgenus Pareas and correspond to the color of icons in Figures 1 and 4. Numbers at tree nodes correspond to PP/UFBS support values, respectively. Photos on thumbnails by N. A. Poyarkov (Pareas abros sp. nov., P. temporalis, and $P$. kuznetsovorum sp. nov.), and P. Pawangkhanant ( $P$. berdmorei truongsonicus ssp. nov. and $P$. carinatus tenasserimicus ssp. nov.). 


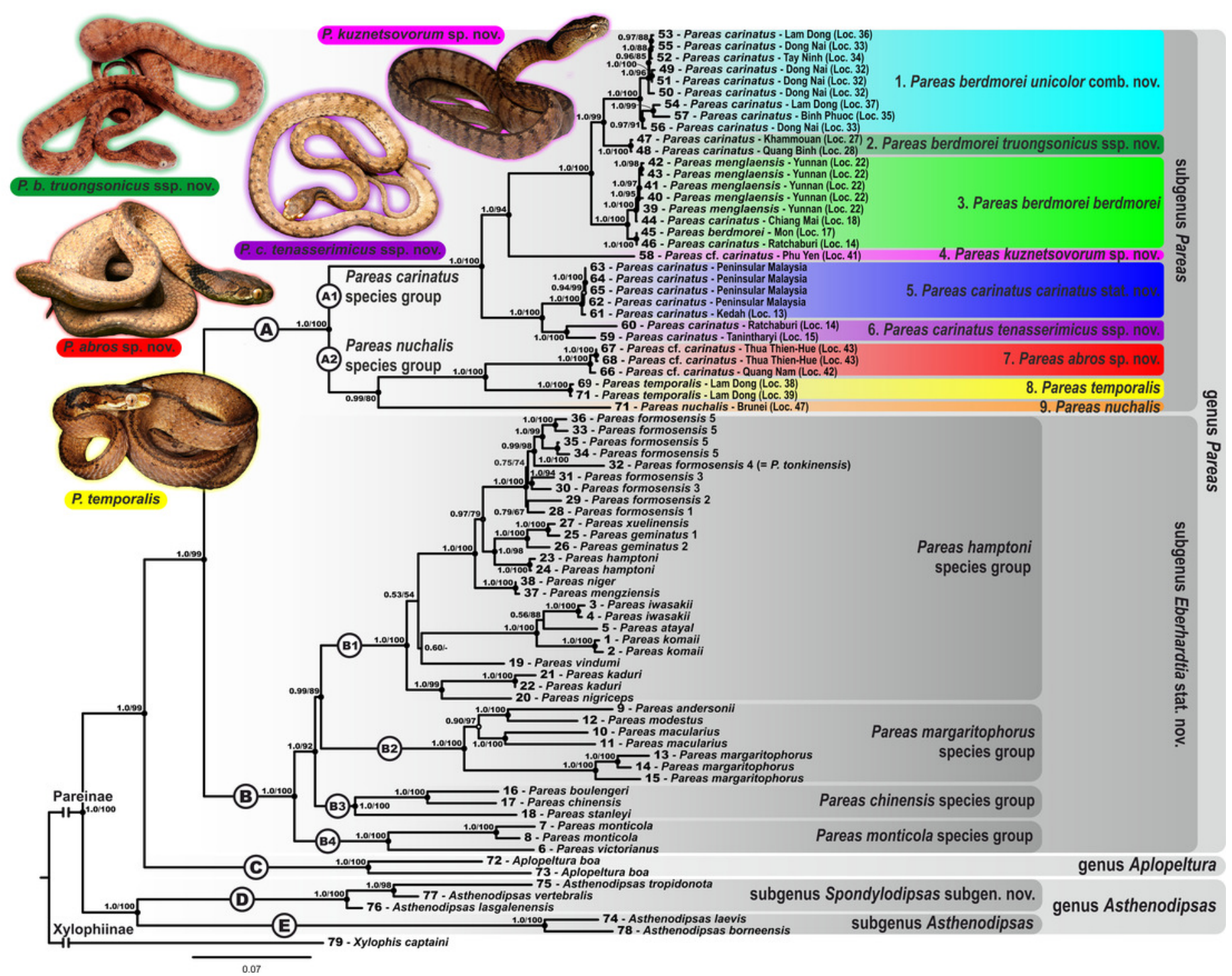


Figure 4

Figure 4. Principal component analysis (PCA) of the species of the subgenus Pareas showing ordination along the first two $(A)$ and the first and the third $(B)$ principal components.

Colors denote the taxa of the subgenus Pareas and correspond to the color of icons in Figures 1 and 3; dot in the center of an icon indicates the holotype or lectotype of a taxon. 

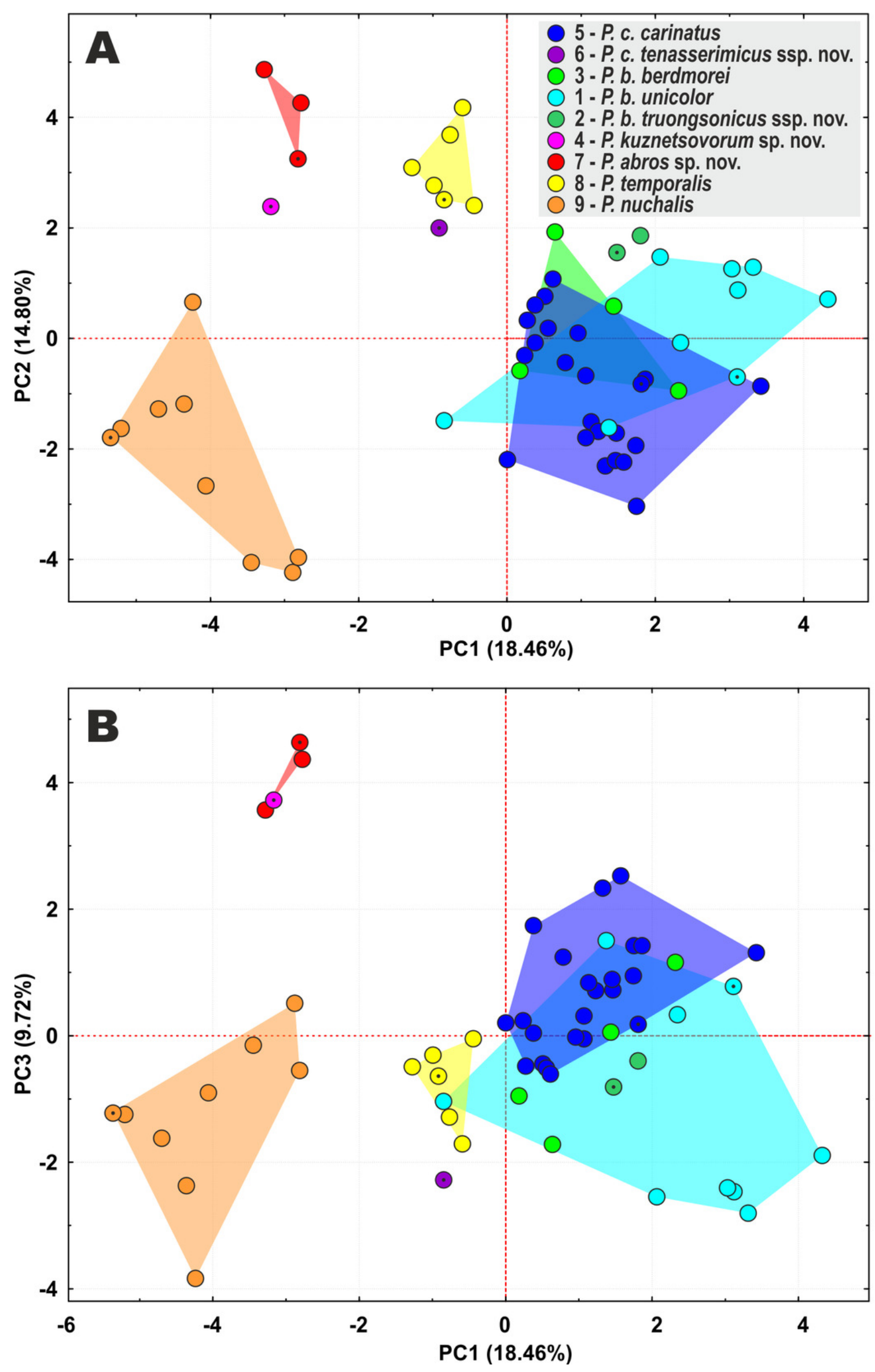


\section{Figure 5}

Figure 5. Head scalation of the genera of the subfamily Pareinae.

Dorsal aspect: A - Pareas (Eberhardtia) formosensis (FMNH 2555567); B - Pareas (Pareas) carinatus (RMNH 954C, lectotype); Ventral aspect: C - Asthenodipsas (Asthenodipsas) malaccana (SMF 32580); D - Asthenodipsas (Asthenodipsas) laevis (SMF 81195); E Asthenodipsas (Spondylodipsas subgen. nov.) vertebralis (ZMB 65285); $\mathrm{F}$ - Asthenodipsas (Spondylodipsas subgen. nov.) tropidonota (RMNH 4902B, lectotype); G - Aplopeltura boa (ZMB 5397); H - Pareas (Pareas) carinatus (ZMB 5397); I - Pareas (Eberhardtia) formosensis (ZMB 30585); J - Pareas (Eberhardtia) margaritophorus (ZMB 6339). Not to scale. Magenta, cyan, blue, red, green and yellow denote frontal, parietals, mental, inframaxillary, infralabials, and chin shields, respectively. Drawings by N. A. Poyarkov (A-B) and L. B. Salamakha (C-J). 


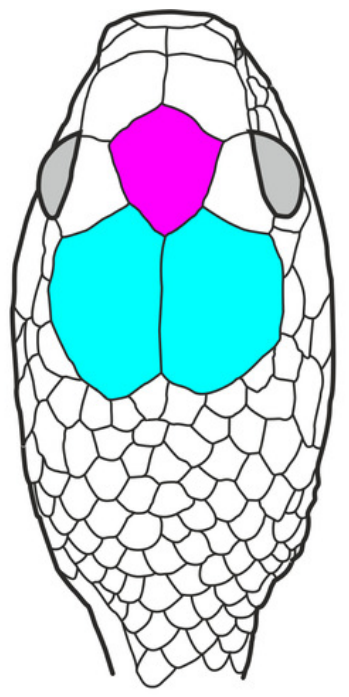

$\triangle$

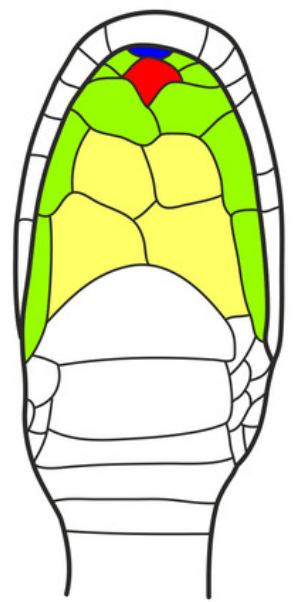

C

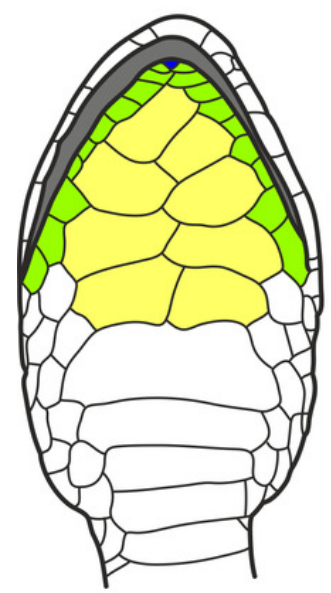

G

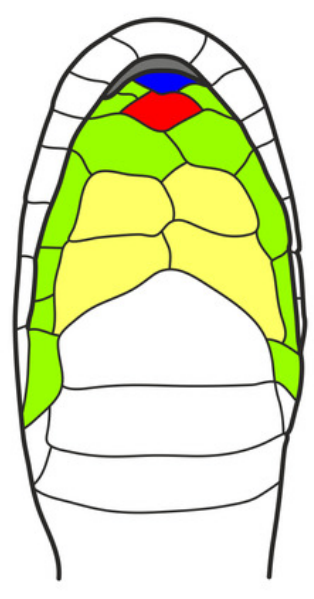

D

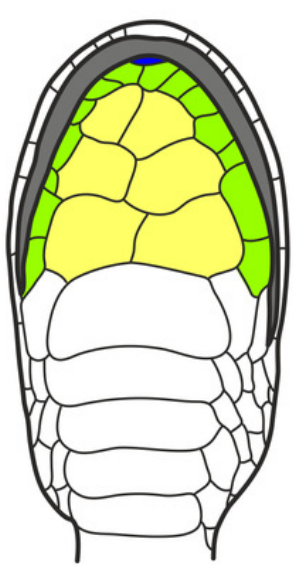

H

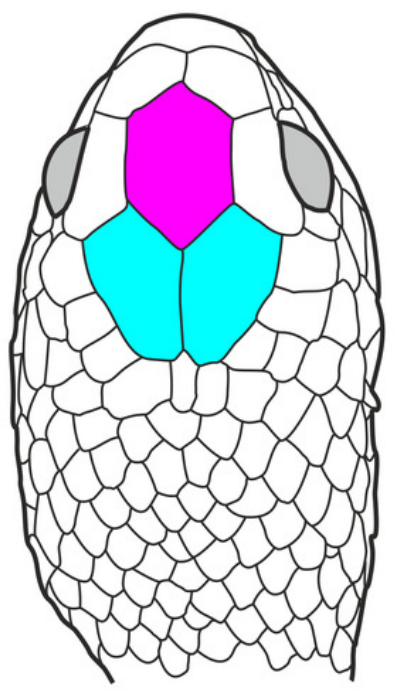

B
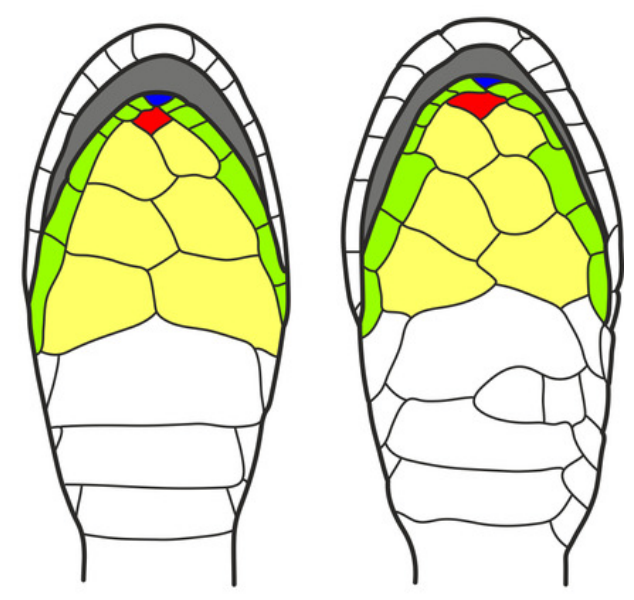

$\mathbf{E}$
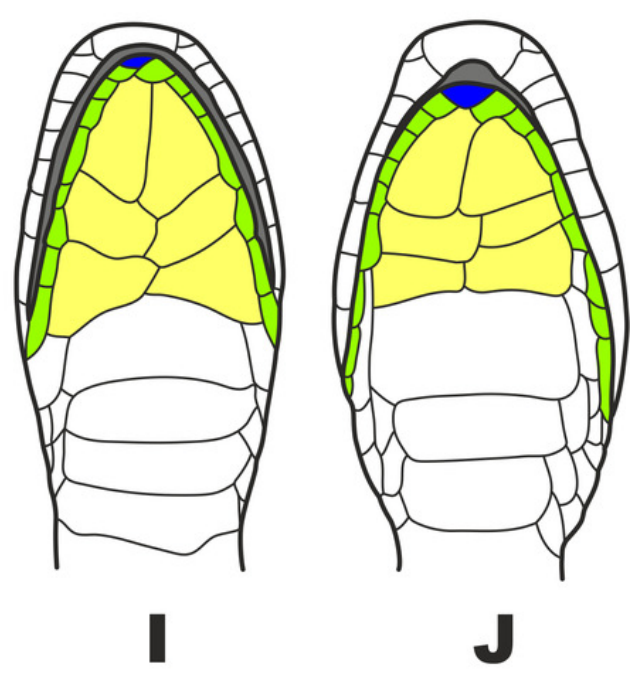

frontal $\square$ parietals $\quad \square$ chin shields mental $\square$ inframaxillary $\square$ infralabials

Peer) reviewing PDF | (2021:08:64561:2:0:NEW 8 Dec 2021) 


\section{Figure 6}

Figure 6. Lateral views of head scalation of taxa of the subgenus Pareas.

A - lectotype of Pareas carinatus Wagler, 1830 (RMNH 954 C); B - holotype of $P$. carinatus tenasserimicus ssp. nov. (ZMMU R-16800); C - lectotype of Pareas berdmorei Theobald, 1868 (ZSI 8022); D - holotype of Pareas menglaensis Wang, Che, Liu, Ki, Jin, Jiang, Shi \& Guo, 2020 (YBU 14124); E - holotype of P. berdmorei truongsonicus ssp. nov. (ZMMU R-16801); F - holotype of Amblycephalus carinatus unicolor Bourret, 1934 (MNHN 1938.0149); G holotype of Pareas kuznetsovorum sp. nov. (ZMMU R-16802); H - Pareas nuchalis (Boulenger, 1900) (FMNH 131635); I - holotype of Pareas abros sp. nov. (ZMMU R-16393); J male of Pareas temporalis Le, Tran, Hoang \& Stuart, 2021 (ZMMU R-13656). Not to scale. Drawings by L. B. Salamakha. 

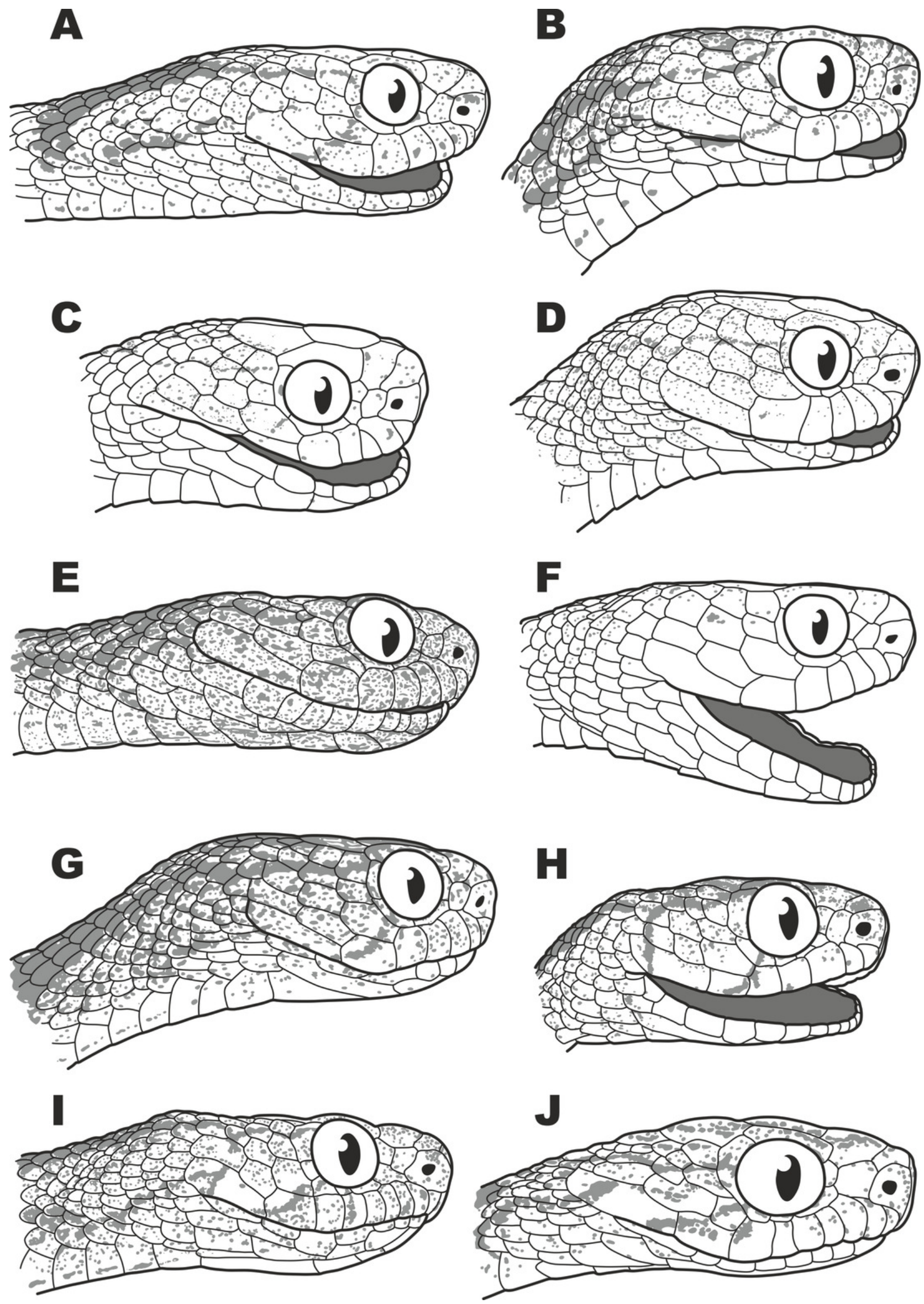

Peerj reviewing PDF | (2021:08:64561:2:0:NEW 8 Dec 2021) 
Figure 7

Figure 7. Lectotype of Pareas carinatus Wagler, 1830 in preservative (RMNH 954 C, adult male).

A - dorsal view of body; B - ventral view of body; C-F - head in lateral right, lateral left, dorsal, and ventral aspects, respectively. Photos by G. Vogel. 


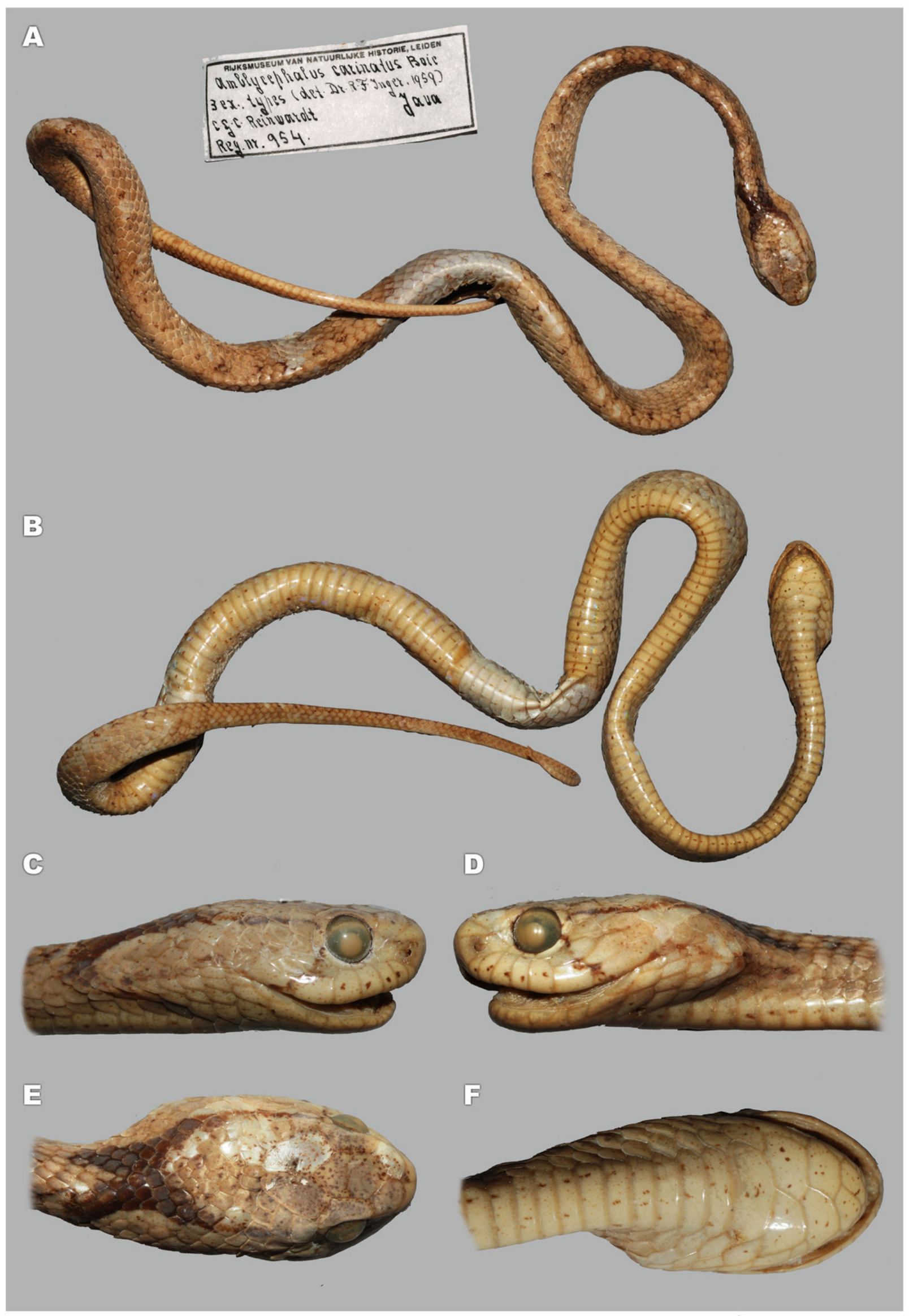


Figure 8

Figure 8. Holotype of $P$. carinatus tenasserimicus ssp. nov. in preservative (ZMMU R-16800, adult male).

A - dorsal view of body; B - ventral view of body; C-F - head in lateral right, lateral left, dorsal, and ventral aspects, respectively. Photos by N. A. Poyarkov. 

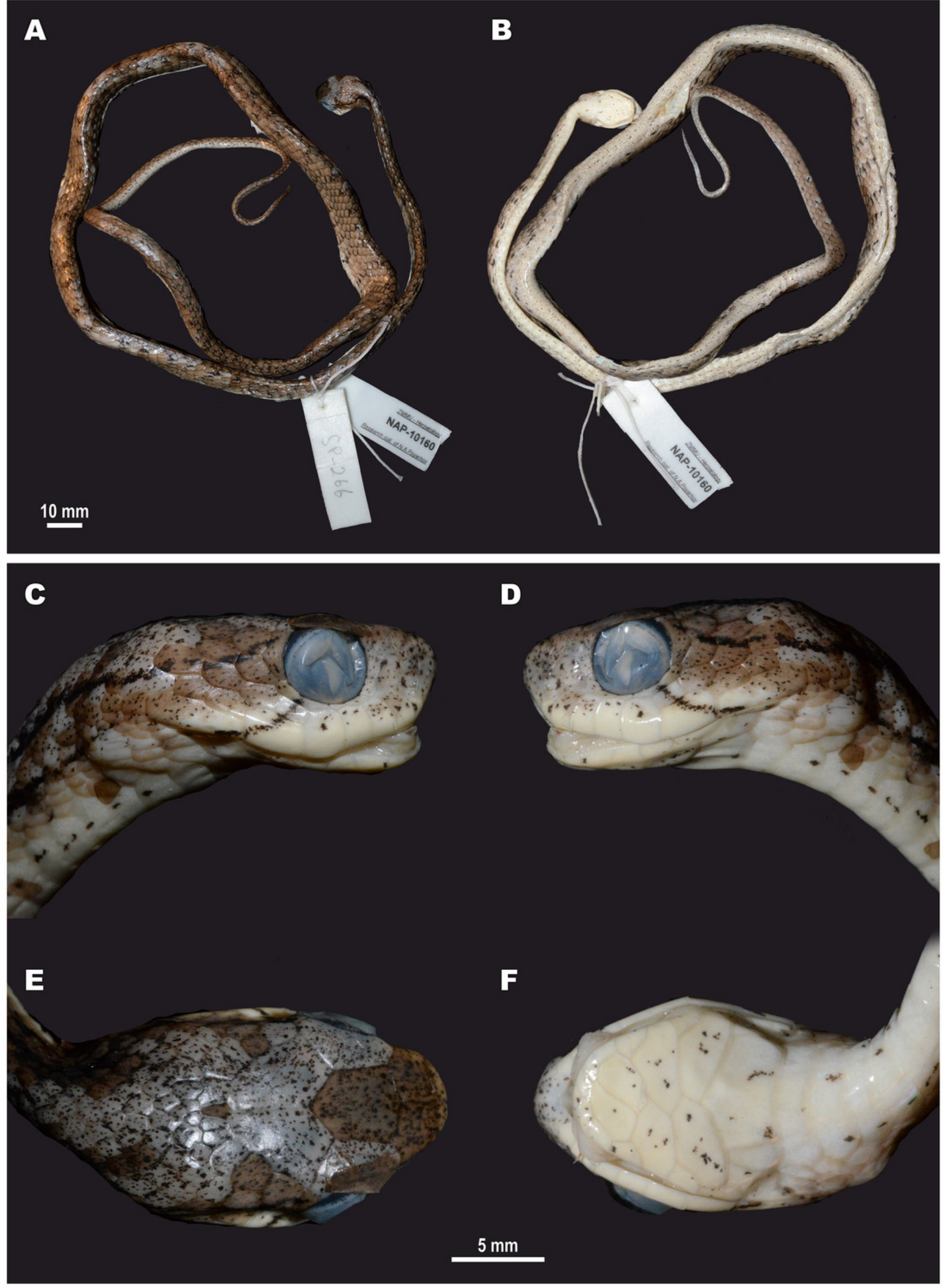


\section{Figure 9}

Figure 9. Members of the Pareas carinatus complex in life.

A - P. c. carinatus from Hala-Bala W.S., Narathiwat Province, Thailand; B, C - P. c. carinatus from Gunung Leuser N.P., Bukit Lawang Province, Sumatra, Indonesia; D - P. c. cf. tenasserimicus ssp. nov. from Kaeng Krachan N.P., Phetchaburi Province, Thailand; E - P. c. tenasserimicus ssp. nov. from Suan Phueng, Ratchaburi Province, Thailand. Photos by L.A. Neimark (A), Guek Hock Ping aka Kurt Orion (B), H.X.N. Nguyen (C), P. Pawangkhanant (D), and M. Naiduangchan (E). 

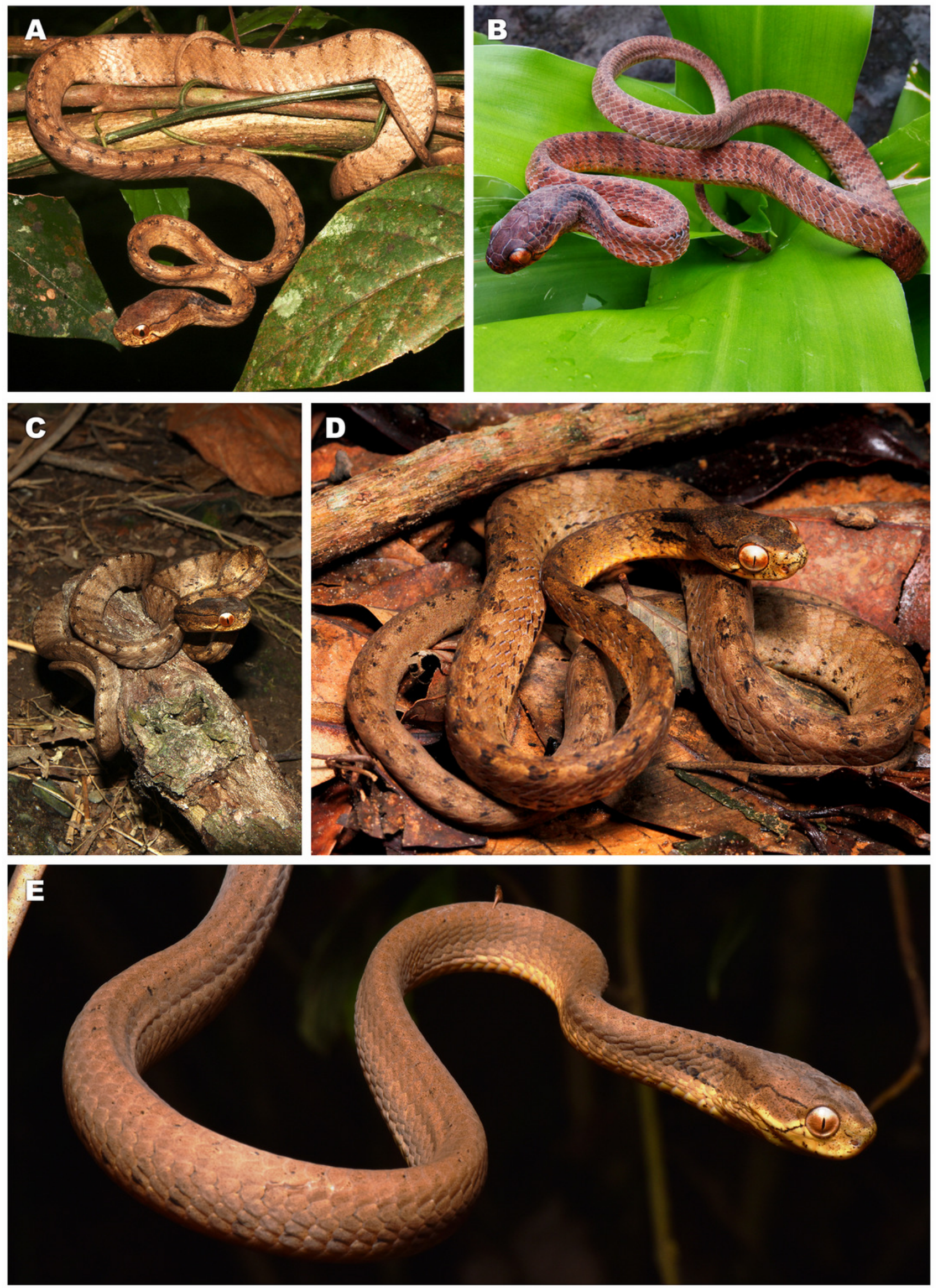
Figure 10

Figure 10. Lectotype of Pareas berdmorei Theobald, 1868 in preservative (ZSI 8022, adult male).

A - general dorsolateral view of body; B - lateral left aspect of head. Photos by I. Das. 

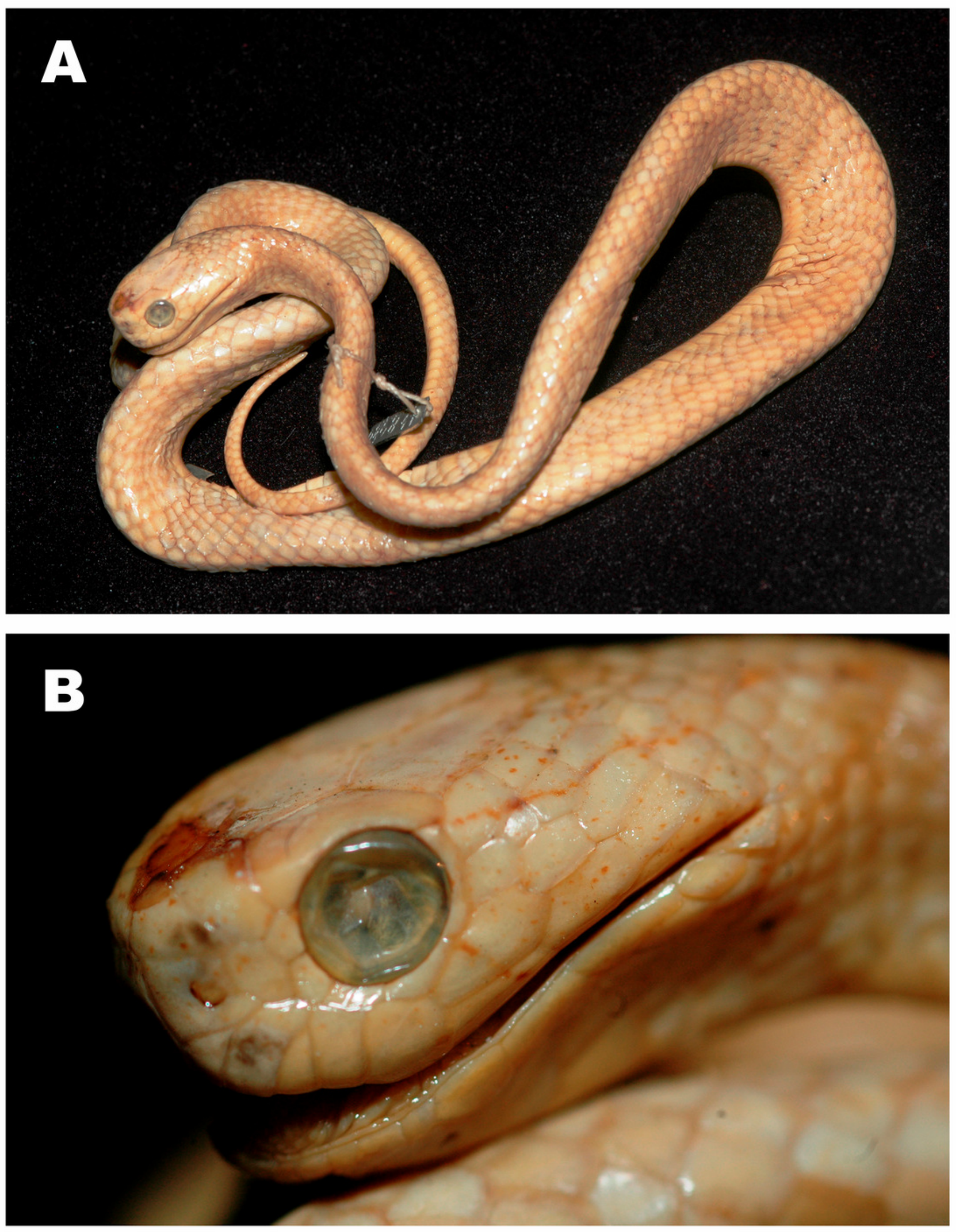
Figure 11

Figure 11. Holotype of Amblycephalus carinatus unicolor Bourret, 1934 in preservative (MNHN 1938.0149, adult female).

A - dorsal view of body; B - ventral view of body; C-F - head in lateral right, lateral left, dorsal, and ventral aspects, respectively. Photos by G. Vogel. 

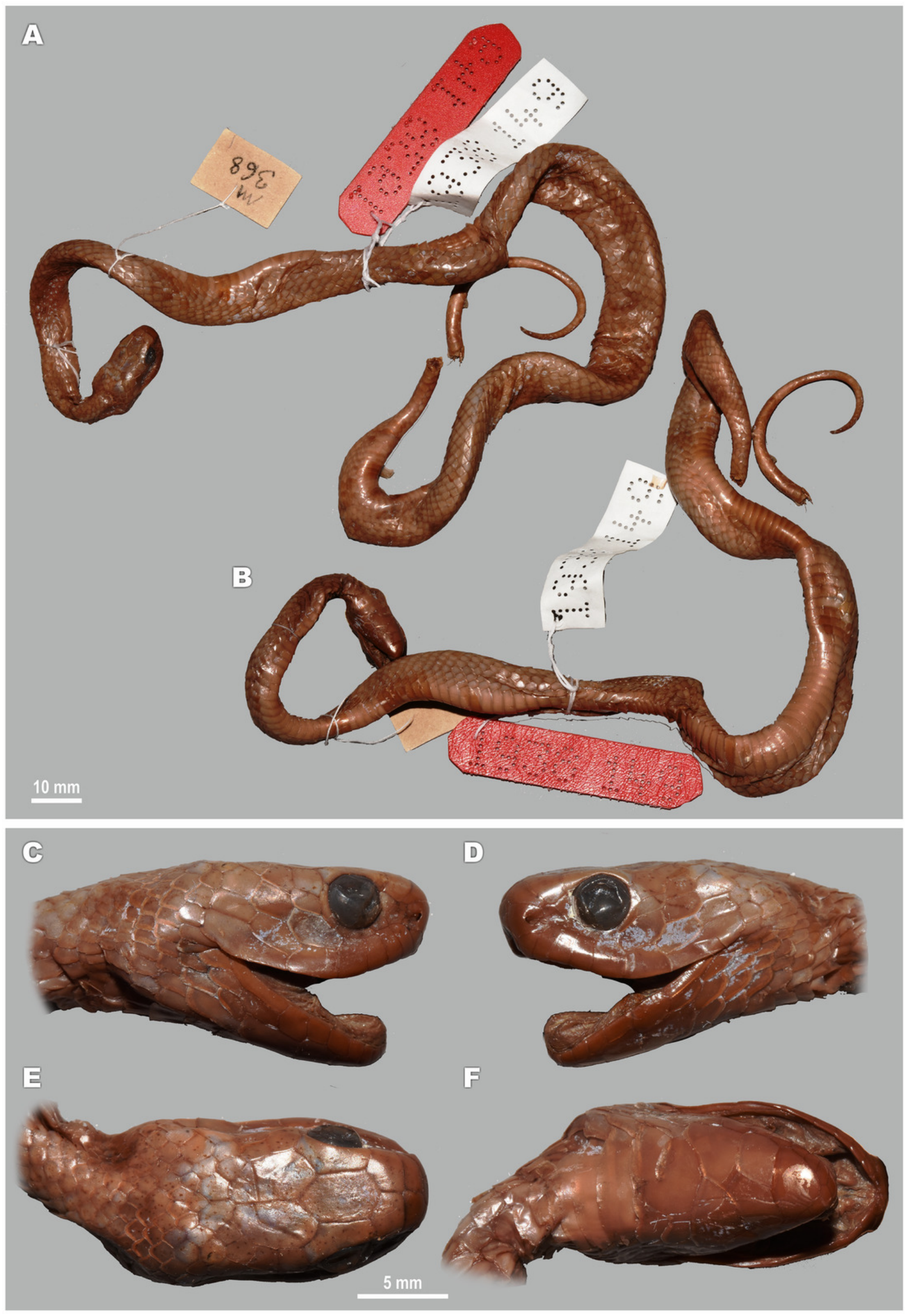


\section{Figure 12}

Figure 12. Holotype of $P$. berdmorei truongsonicus ssp. nov. in preservative (ZMMU R-16801, adult female).

A - dorsal view of body; B - ventral view of body; C-F - head in lateral right, lateral left, dorsal, and ventral aspects, respectively. Photos by N. A. Poyarkov. 

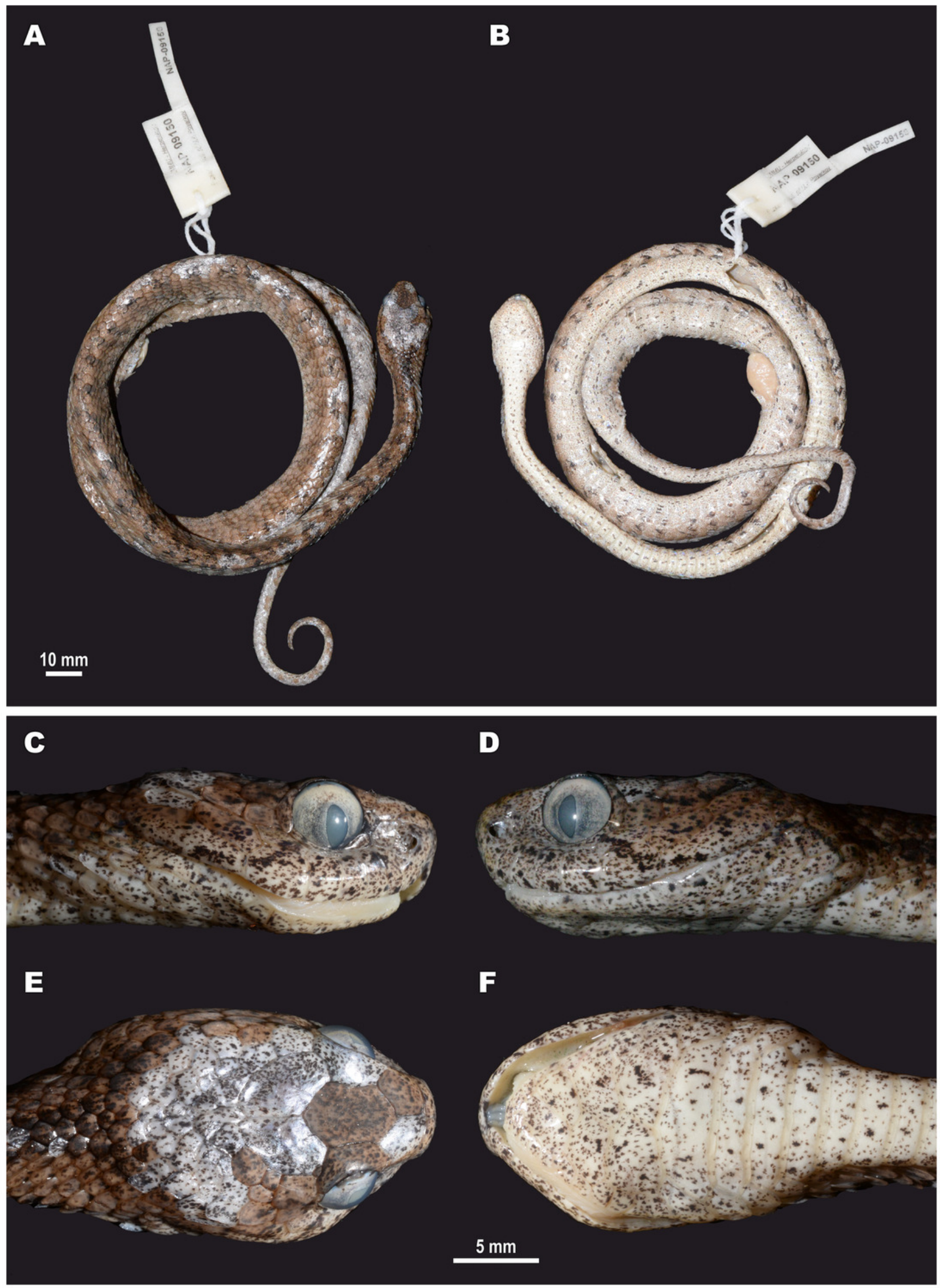


\section{Figure 13}

Figure 13. Members of the Pareas berdmorei complex in life.

A - P. b. berdmorei from Thung Yai Naresuan W.S., Kanchanaburi Province, Thailand; B - P. b. berdmorei from Huai Kha Khaeng W.S., Uthai Thani Province, Thailand; C - P. b. berdmorei from Phu Hin Rong Kla N.P., Phitsanulok Province, Thailand; D - P. b. berdmorei from Jiangcheng, Pu'er City, Yunnan Province, China (close to the type locality of $P$. menglaensis); E - P. b. unicolor from Cat Tien N.P., Dong Nai Province, Vietnam; F - P. b. unicolor from Loc Bac Forest, Lam Dong Province, Vietnam; G - P. berdmorei cf. truongsonicus ssp. nov. from Xe Pian N.P.A., Champasak Province, Laos; H - P. berdmorei truongsonicus ssp. nov. from Nahin District, Khammouan Province, Laos (ZMMU R-16801, holotype in life). Photos by P. Pawangkhanant (A-B, H), N.A. Poyarkov (C, E-F), and G. Vogel (D, G). 

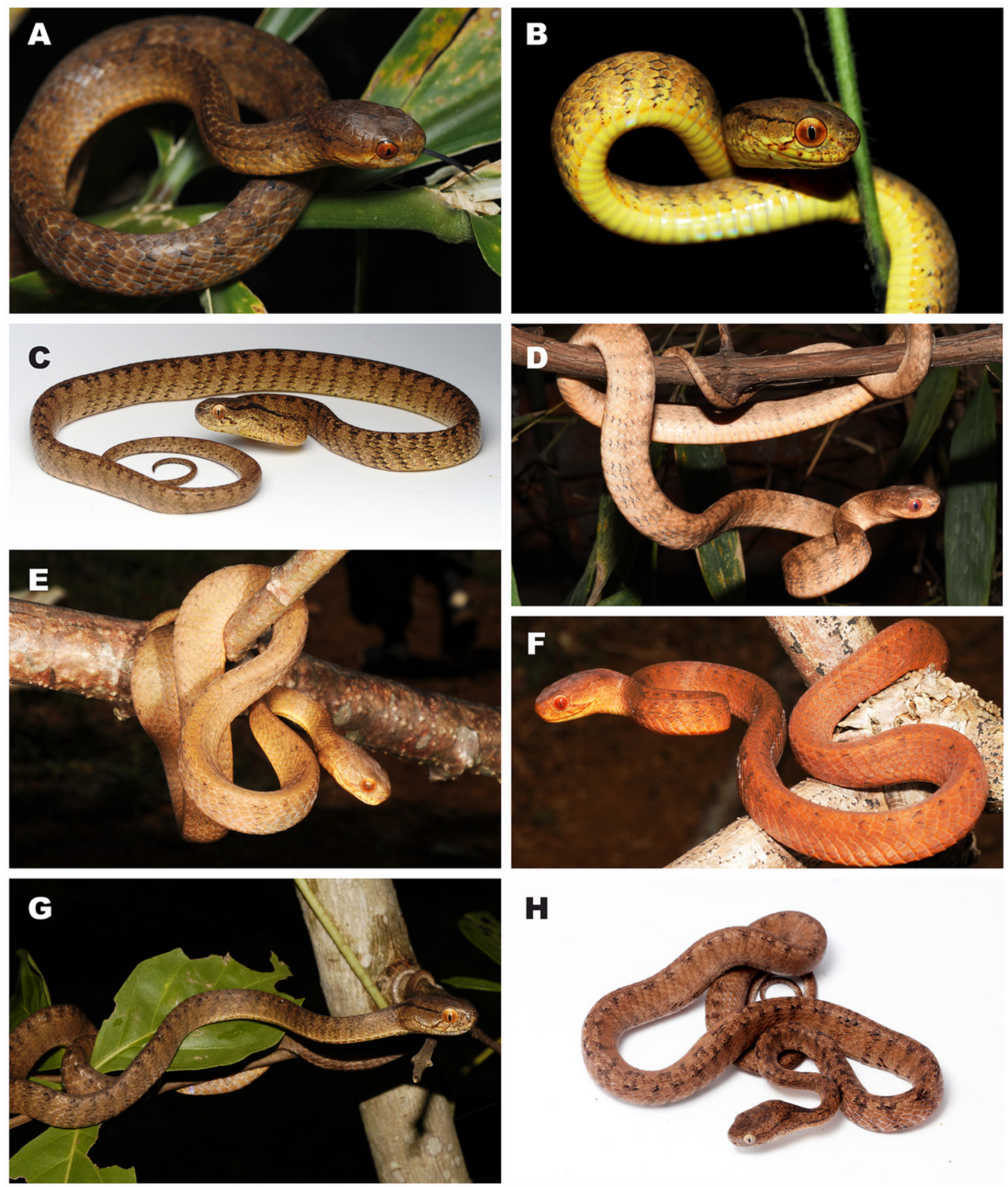

H

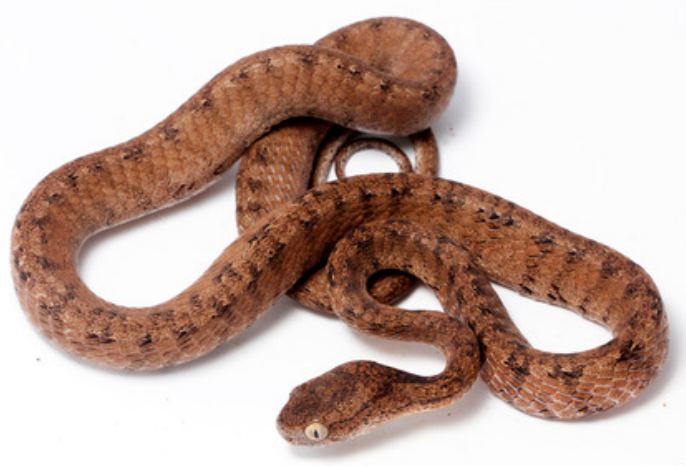




\section{Figure 14}

Figure 14. Holotype of Pareas kuznetsovorum sp. nov. in life (ZMMU R-16802, adult male).

A - dorsal view of body; B - ventral view of body; C-F - head in lateral right, lateral left, dorsal, and ventral aspects, respectively. Photos by N. A. Poyarkov. 

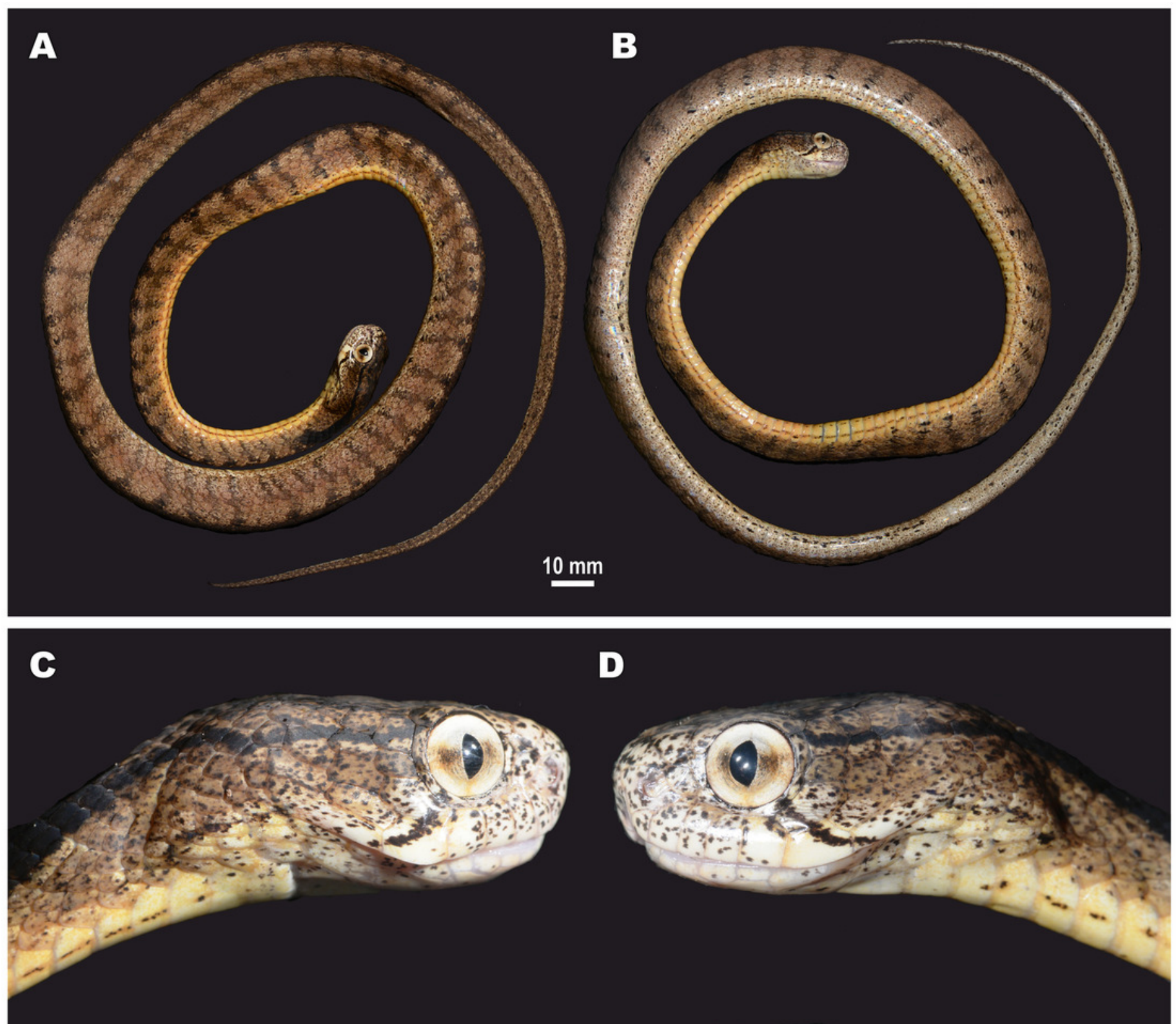

D
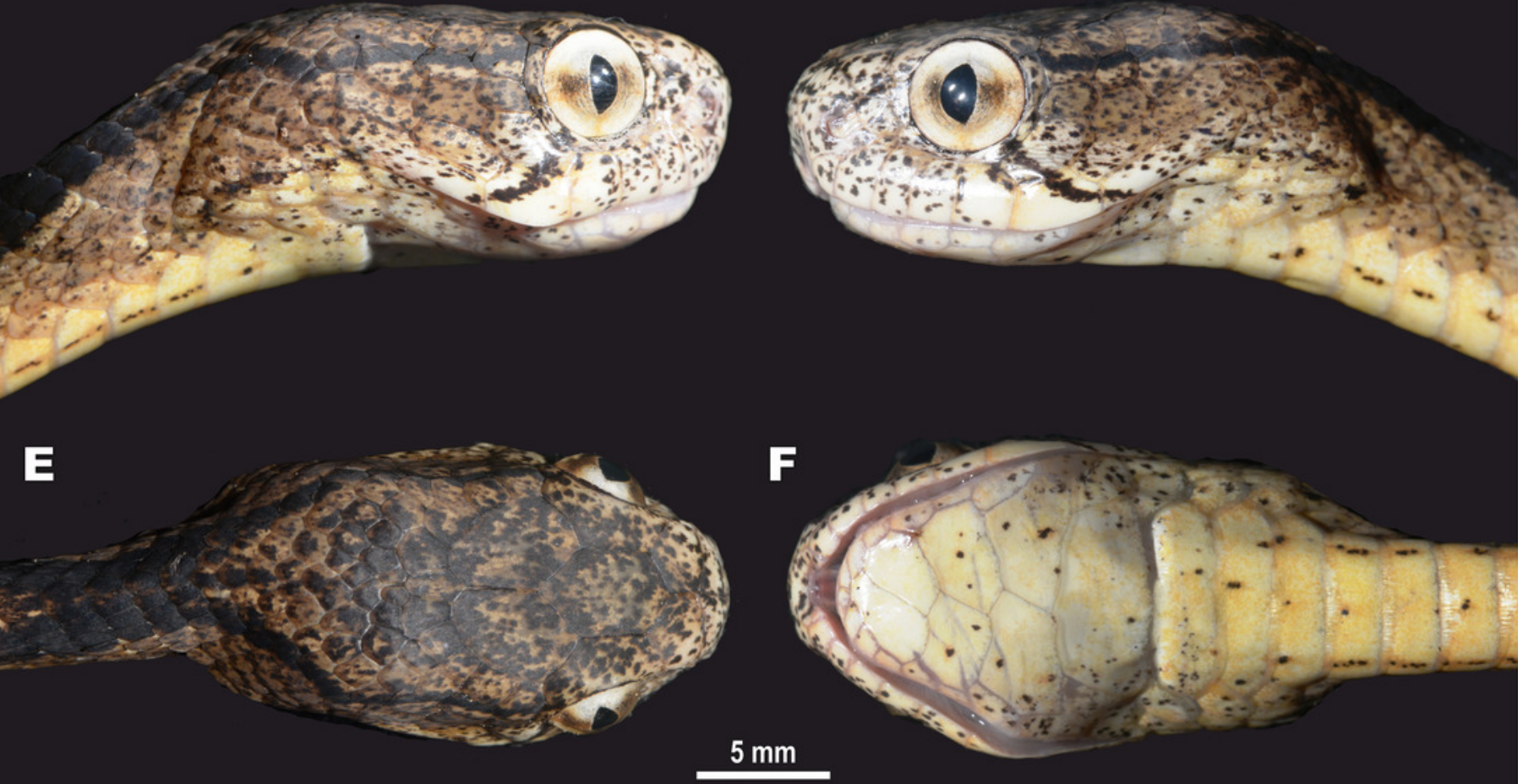


\section{Figure 15}

Figure 15. Holotype of Pareas kuznetsovorum sp. nov. in life (ZMMU R-16802, adult male) from Song Hinh, Phu Yen Province, Vietnam.

A - general view; B-C - sulcal and asulcal aspects of fully everted hemipenis; D - close-up of midbody showing smooth dorsal scales. Photos by N. A. Poyarkov. 

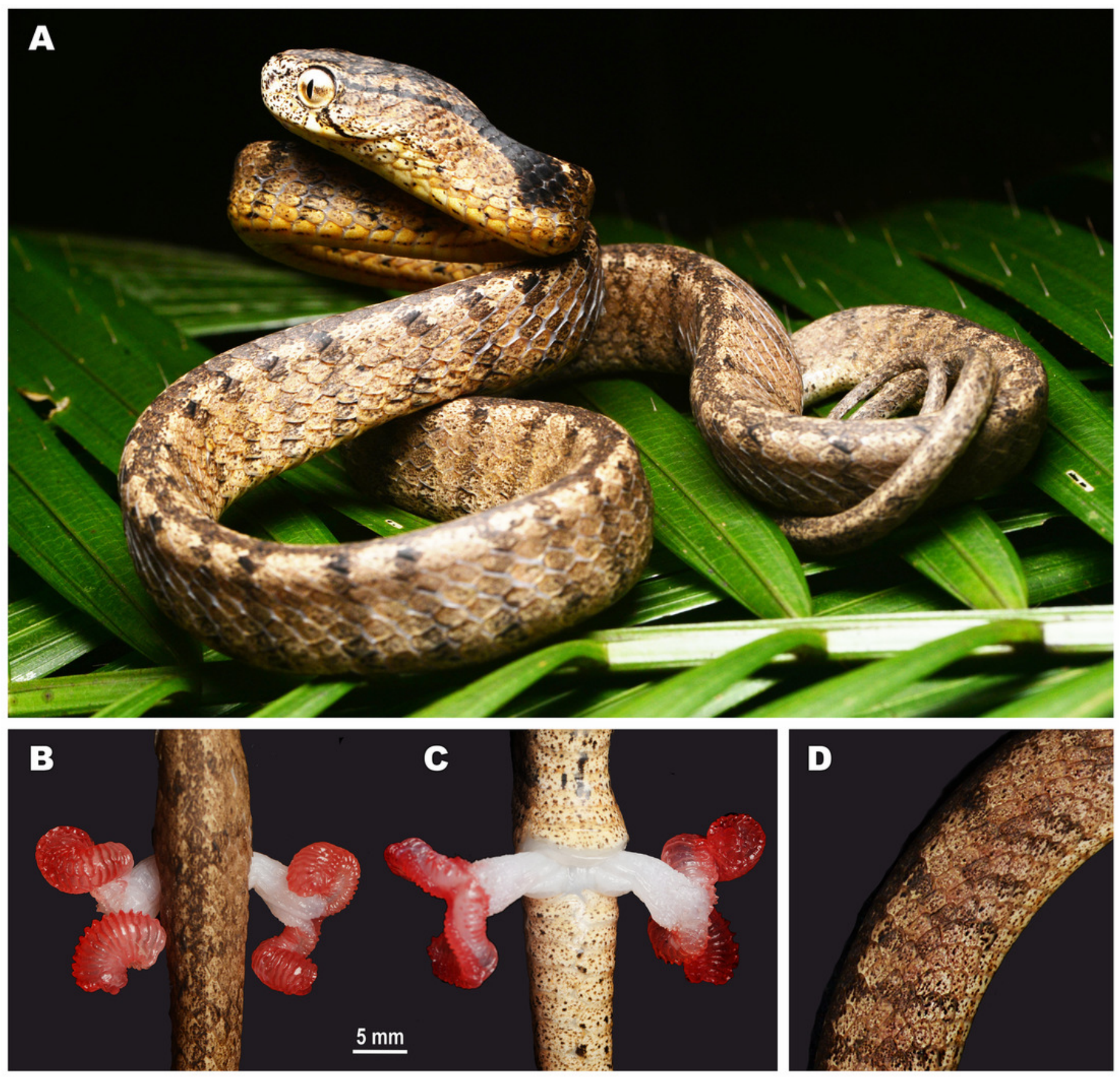


\section{Figure 16}

Figure 16. Holotype of Amblycephalus nuchalis Boulenger, 1900 in preservative (NHMUK 1901.5.14.2, adult male).

A - dorsolateral view of body; B - ventrolateral view of body; C-F - head in lateral right, lateral left, dorsal, and ventral aspects, respectively. Photos by G. Vogel. 


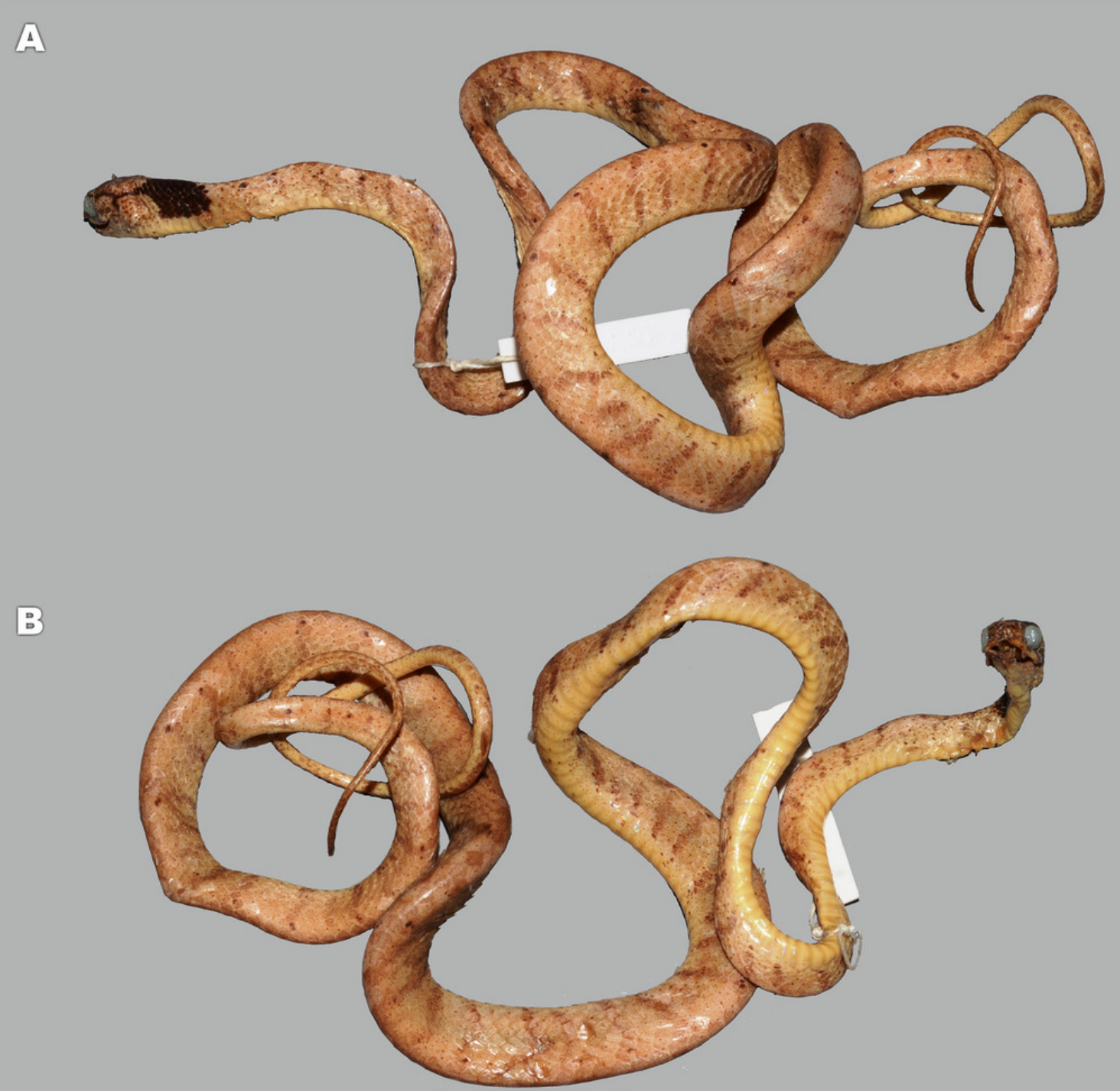

G

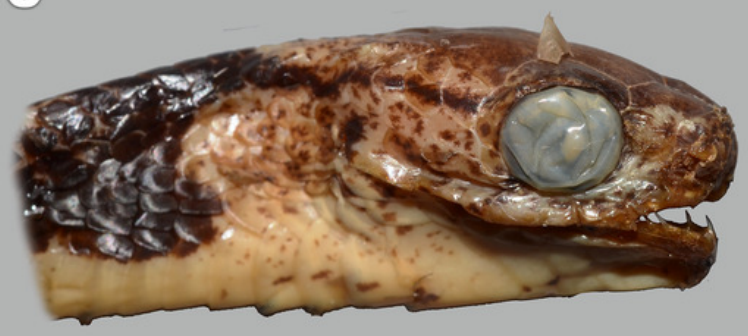

틀

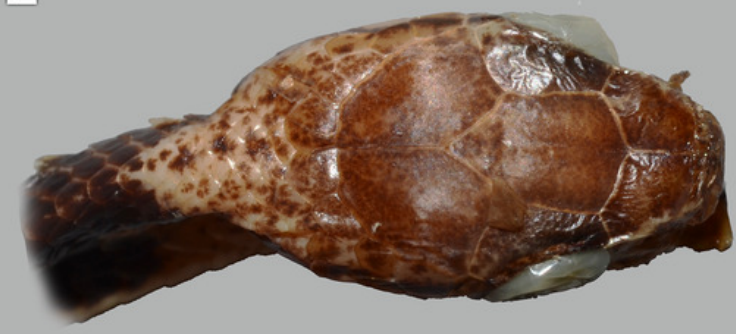

D

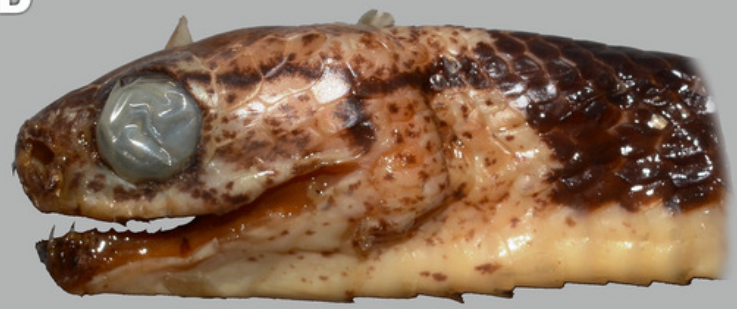

F

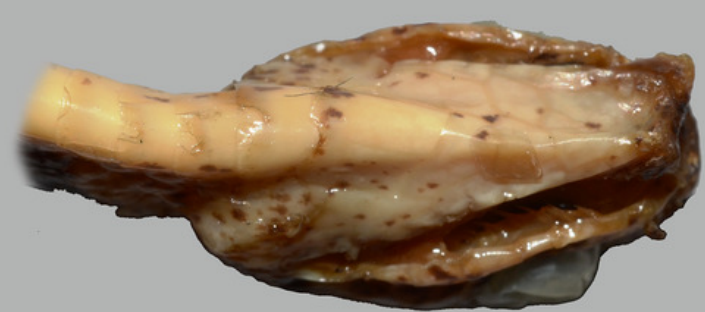




\section{Figure 17}

Figure 17. Pareas nuchalis in life, adult male from Kota Kinabalu N.P., Kundasang, Sabah, Borneo, Malaysia.

Photo by L.A. Neimark.

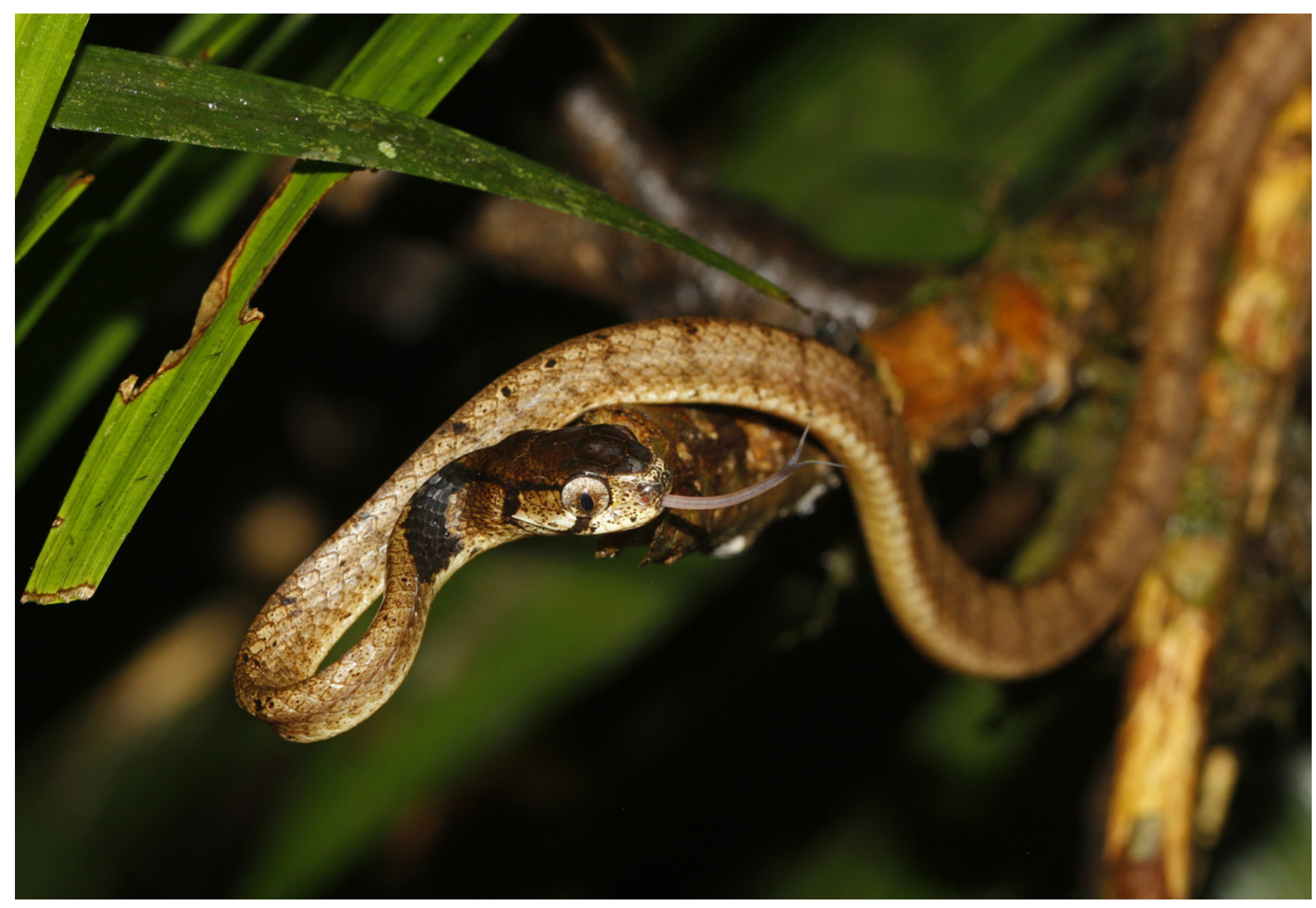




\section{Figure 18}

Figure 18. Holotype of Pareas abros sp. nov. in life (ZMMU R-16393, adult male).

A - lateral left view of body; B - later right view of body; C-E - head in lateral right, ventral, and dorsal aspects, respectively; F - close-up of midbody showing keeled dorsal scales (in eleven rows, five to six of them seen on one side of the body). Photos by N. A. Poyarkov. 

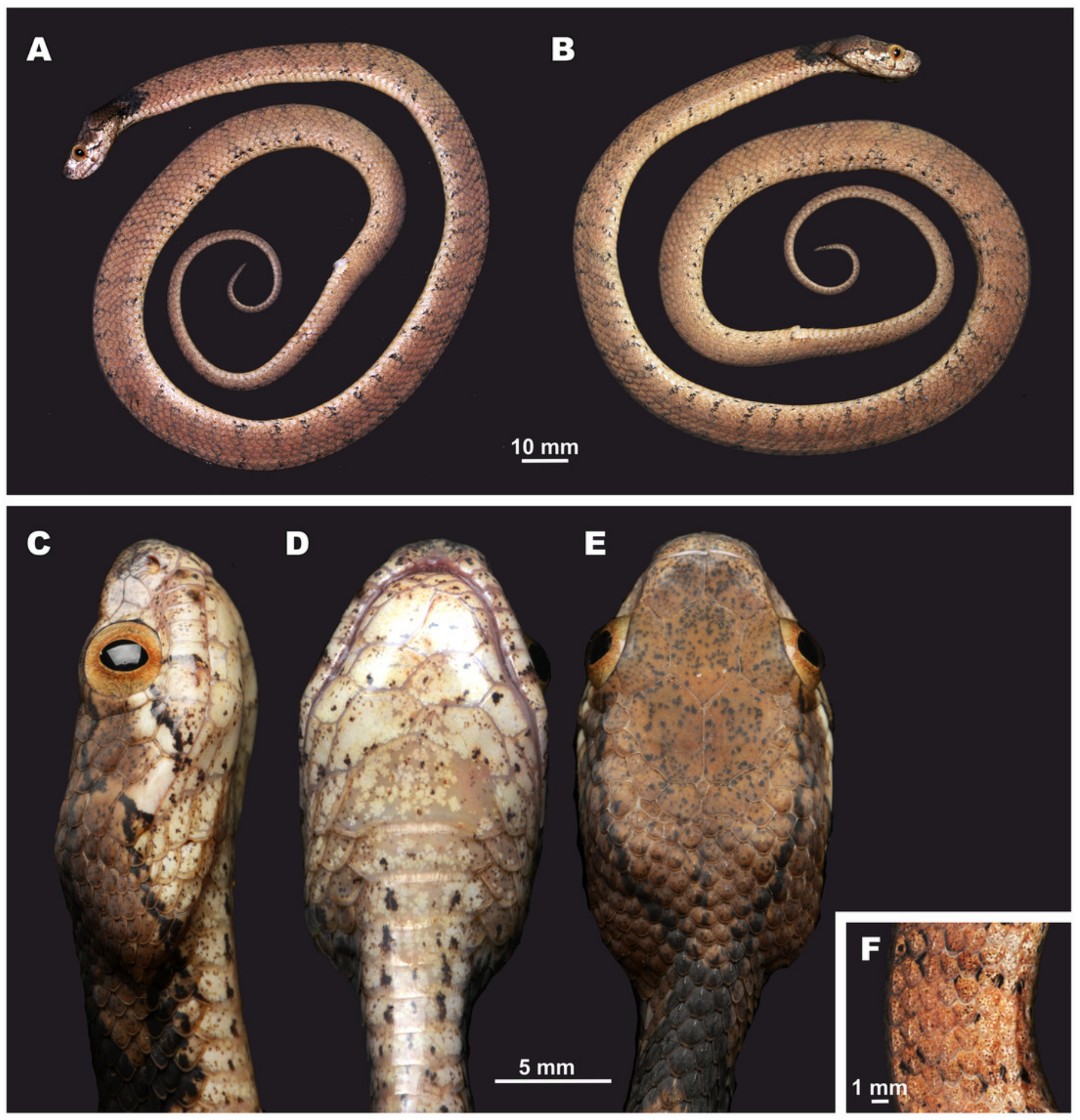


\section{Figure 19}

Figure 19. Pareas abros sp. nov. in life.

A - male holotype from Song Thanh N.P., Quang Nam Province, Vietnam (ZMMU R-16393); B

- male paratype from A Roang, Sao La N.R., Thua Thien - Hue Province, Vietnam (ZMMU R-16392); C - female paratype from A Roang, Sao La N.R., Thua Thien - Hue Province, Vietnam (ZMMU R-14788); D - partially everted hemipenis of ZMMU R-16392 from asulcal (above) and sulcal (below) aspects; E - close-up of midbody of ZMMU R-16392 showing enlarged vertebrals and keeled dorsal scales (in eleven rows). Photos by N. A. Poyarkov.
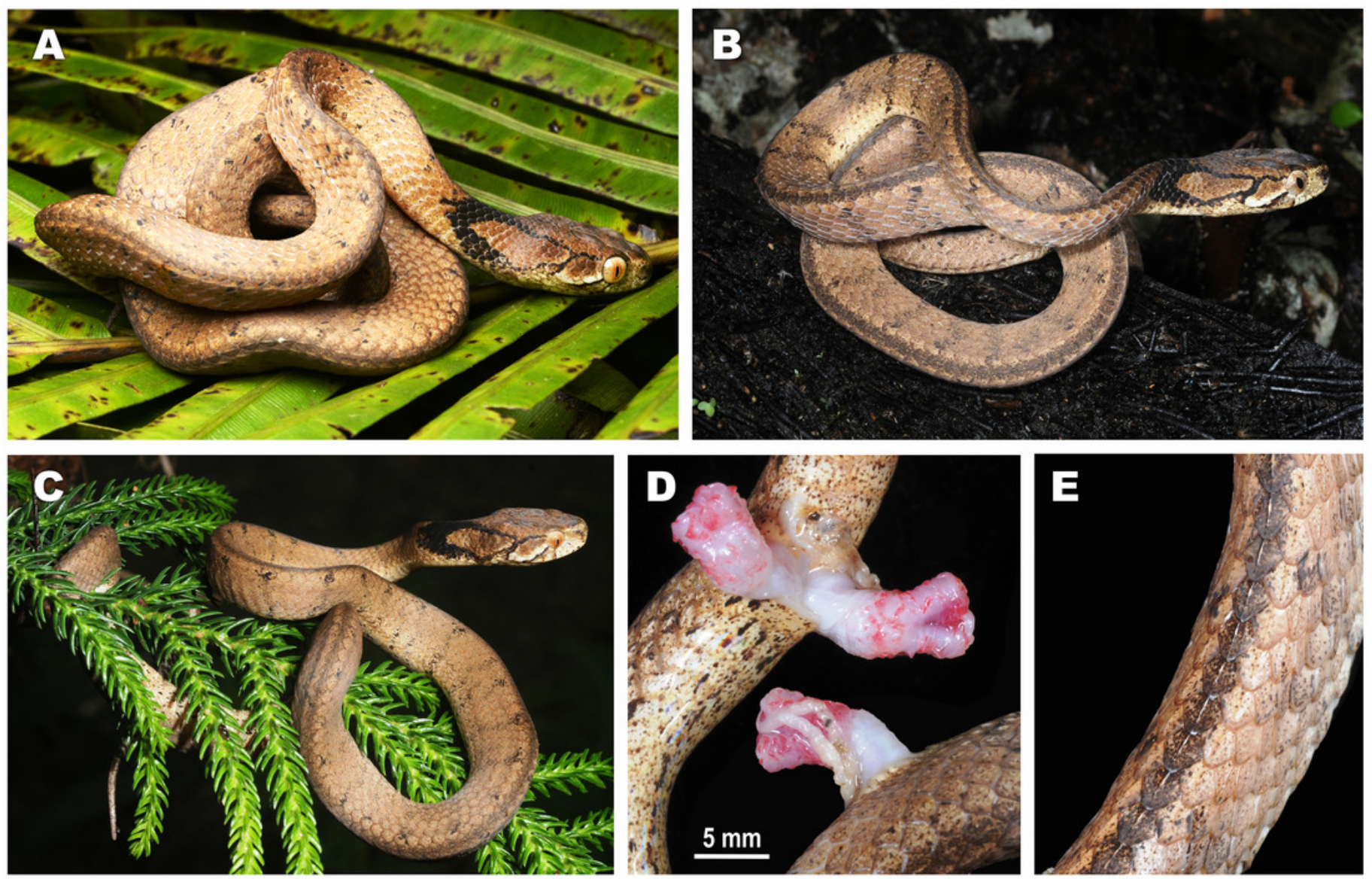
Figure 20

Figure 20. Specimen of Pareas temporalis Le, Tran, Hoang \& Stuart, 2021 in preservative (ZMMU R-13656, adult male).

A - dorsal view of body; B - ventral view of body; C-E - head in lateral right, ventral, and dorsal aspects, respectively. Photos by G. Vogel. 

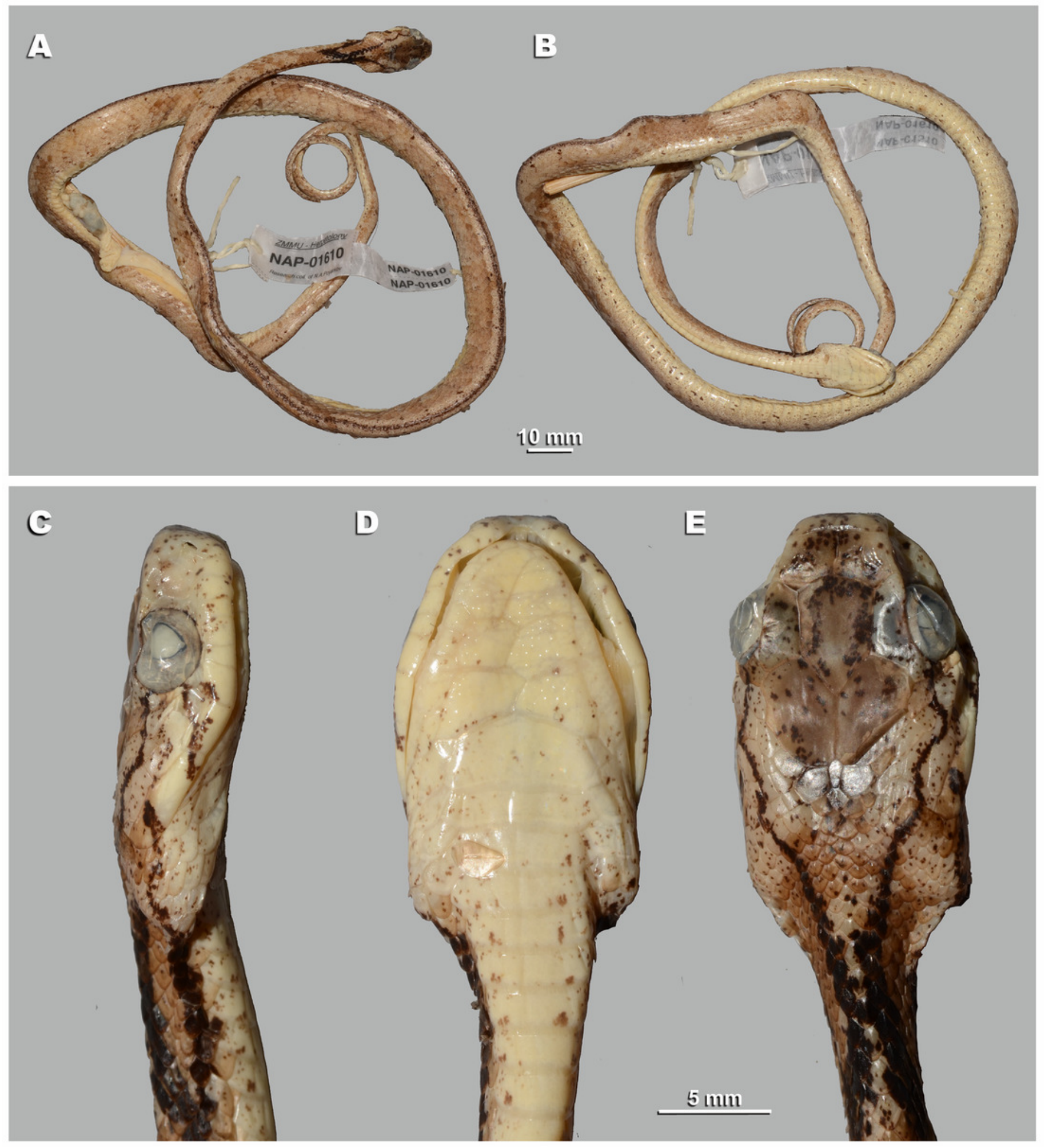
Figure 21

Figure 21. Pareas temporalis in life.

A - male specimen from Cat Loc, Lam Dong Province, Vietnam (ZMMU R-13656); B-C female specimen from Di Linh, Lam Dong Province, Vietnam (SIEZC 20214); D - close-up of midbody of SIEZC 20214 showing all dorsal scales keeled (in 15 rows). Photos by N. A. Poyarkov (A), and L.H. Nguyen (B-D). 

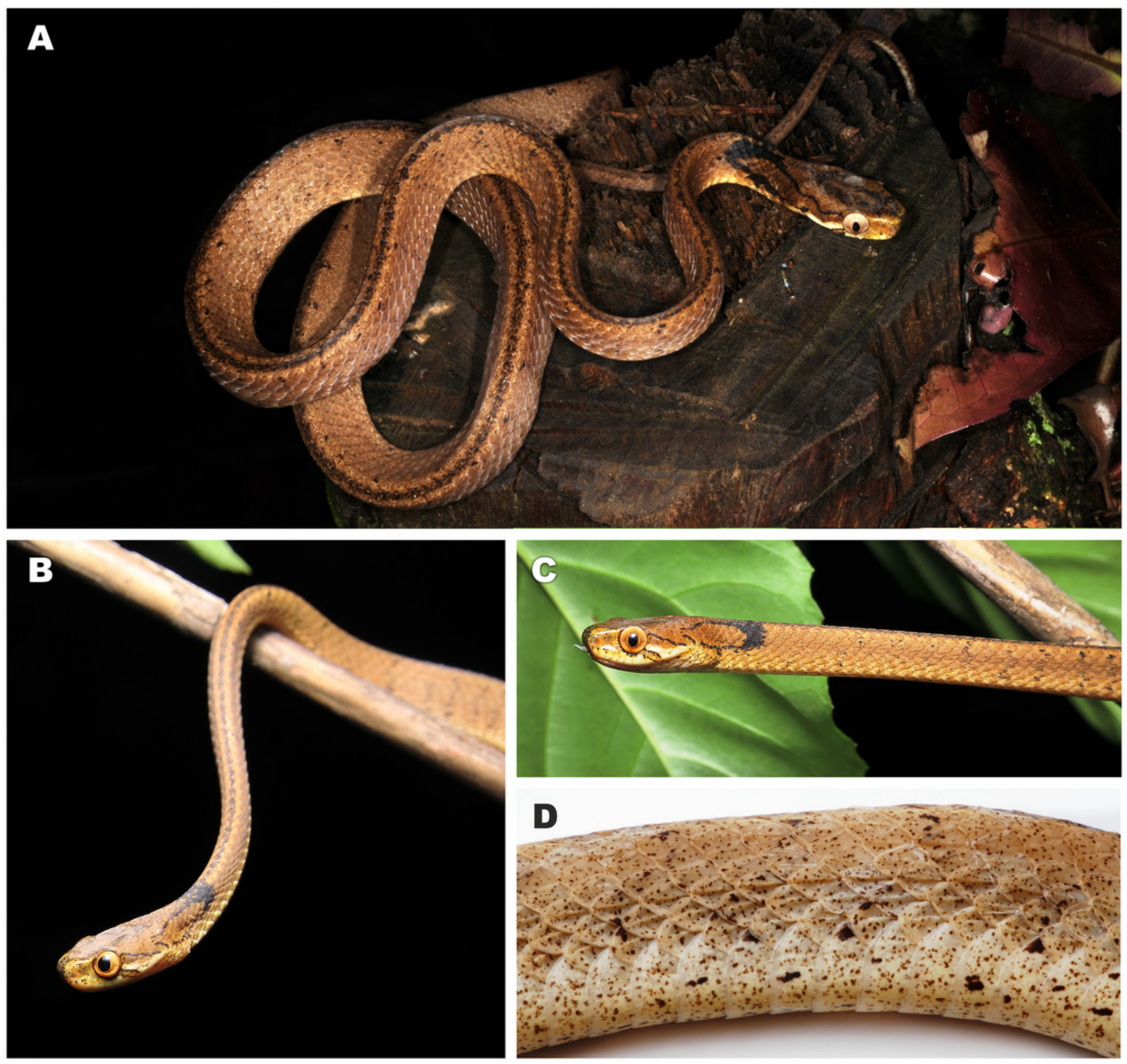